Florida International University FIU Digital Commons

6-18-2013

\title{
Dynamic Image Precompensation for Improving Visual Performance of Computer Users with Ocular Aberrations
}

Jian Huang

jhuan004@fiu.edu

DOI: $10.25148 /$ etd.FI13080516

Follow this and additional works at: https://digitalcommons.fiu.edu/etd

Part of the Graphics and Human Computer Interfaces Commons, and the Signal Processing Commons

\section{Recommended Citation}

Huang, Jian, "Dynamic Image Precompensation for Improving Visual Performance of Computer Users with Ocular Aberrations" (2013). FIU Electronic Theses and Dissertations. 902.

https://digitalcommons.fiu.edu/etd/902 


\title{
FLORIDA INTERNATIONAL UNIVERSITY \\ Miami, Florida
}

\section{DYNAMIC IMAGE PRECOMPENSATION FOR IMPROVING VISUAL PERFORMANCE OF COMPUTER USERS WITH OCULAR ABERRATIONS}

A dissertation submitted in partial fulfillment of the

\author{
requirements for the degree of \\ DOCTOR OF PHILOSOPHY \\ in \\ ELECTRICAL ENGINEERING \\ by \\ Jian Huang
}




\section{To: Dean Amir Mirmiran}

College of Engineering and Computing

This dissertation, written by Jian Huang, and entitled Dynamic Image Precompensation for Improving Visual Performance of Computer Users with Ocular Aberrations, having been approved in respect to style and intellectual content, is referred to you for judgment.

We have read this dissertation and recommend that it be approved.

Malek Adjouadi

Jean H. Andrian

Wei-Chiang Lin

Armando Barreto, Major Professor

Date of Defense: June 18, 2013

The dissertation of Jian Huang is approved.

$\begin{array}{r}\text { Dean Amir Mirmiran } \\ \text { College of Engineering and Computing } \\ \hline \begin{array}{r}\text { Dean Lakshmi N. Reddi } \\ \text { University Graduate School }\end{array}\end{array}$

Florida International University, 2013 
(C) Copyright 2013 by Jian Huang

All rights reserved. 


\section{DEDICATION}

To my beloved wife and parents. 


\section{ACKNOWLEDGMENTS}

I would like to express my deepest appreciation and gratitude to my major professor,

Dr. Armando Barreto, for his patient guidance and consistent encouragement. I would like to acknowledge the Presidential Fellowship from Florida International University, for providing me financial support during my Ph.D. study. I would like to thank my committee members Dr. Malek Adjouadi, Dr. Jean Andrian and Dr. Wei-Chiang Lin, for their insightful advices.

Over the last few years, many individuals have provided me support, friendship and inspiration. Of those I am particularly grateful to Fatemeh Abyarjoo, Jonathan Cofino, Francisco Ortega, Peng Ren and other graduate students at the DSP Lab, with whom I have shared so much good time and enjoyable memories. I would also like to thank Pat Brammer, Ana Saenz and Maria Benincasa, for their enthusiastic support in administration. Finally, and most importantly, I would like to thank my wife, without whose love and encouragement, the completion of this endeavor would never have been possible. 


\author{
ABSTRACT OF THE DISSERTATION \\ DYNAMIC IMAGE PRECOMPENSATION FOR IMPROVING VISUAL \\ PERFORMANCE OF COMPUTER USERS WITH OCULAR ABERRATIONS \\ by \\ Jian Huang \\ Florida International University, 2013 \\ Miami, Florida \\ Professor Armando Barreto, Major Professor
}

With the progress of computer technology, computers are expected to be more intelligent in the interaction with humans, presenting information according to the user's psychological and physiological characteristics. However, computer users with visual problems may encounter difficulties on the perception of icons, menus, and other graphical information displayed on the screen, limiting the efficiency of their interaction with computers.

In this dissertation, a personalized and dynamic image precompensation method was developed to improve the visual performance of the computer users with ocular aberrations. The precompensation was applied on the graphical targets before presenting them on the screen, aiming to counteract the visual blurring caused by the ocular aberration of the user's eye. A complete and systematic modeling approach to describe the retinal image formation of the computer user was presented, taking advantage of modeling tools, such as Zernike polynomials, wavefront aberration, Point Spread Function and Modulation Transfer Function. The ocular aberration of the computer user was originally measured by a wavefront aberrometer, as a reference for the precompensation model. The dynamic precompensation was generated based on the resized aberration, with the real-time pupil diameter monitored. 
The potential visual benefit of the dynamic precompensation method was explored through software simulation, with the aberration data from a real human subject. An "artificial eye" experiment was conducted by simulating the human eye with a high-definition camera, providing objective evaluation to the image quality after precompensation. In addition, an empirical evaluation with 20 human participants was also designed and implemented, involving image recognition tests performed under a more realistic viewing environment of computer use. The statistical analysis results of the empirical experiment confirmed the effectiveness of the dynamic precompensation method, by showing significant improvement on the recognition accuracy. The merit and necessity of the dynamic precompensation were also substantiated by comparing it with the static precompensation. The visual benefit of the dynamic precompensation was further confirmed by the subjective assessments collected from the evaluation participants. 


\section{TABLE OF CONTENTS}

CHAPTER

PAGE

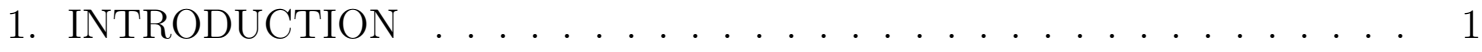

1.1 Problem Statement . . . . . . . . . . . . . . . . . . . . . . . . . 1

1.2 Objective of Study . . . . . . . . . . . . . . . . . . . . 2

1.3 Significance of Study . . . . . . . . . . . . . . . . . . . 3

1.4 Literature Review . . . . . . . . . . . . . . . . . . . . . . . . 5

1.5 Dissertation Structure . . . . . . . . . . . . . . . . . . 9

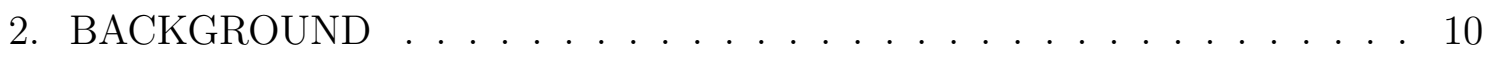

2.1 Human Vision System . . . . . . . . . . . . . . . . . . . . 10

2.1 .1 Cornea . . . . . . . . . . . . . . . . . . . . . . . . . . 11

2.1.2 Lens and Accommodation . . . . . . . . . . . . . . . . . . . 12

2.1 .3 Iris and Pupil Response . . . . . . . . . . . . . . . . . . . . 12

2.1.4 Photoreceptors . . . . . . . . . . . . . . . . . . . . . . . . 14

2.2 Geometrical and Wave Optics . . . . . . . . . . . . . . . . . . . 14

2.2 .1 Reflection and Refraction . . . . . . . . . . . . . . . . . . 14

2.2.2 Paraxial Theory and Refractive Error . . . . . . . . . . . . . . . . . 16

2.2 .3 Wavefront Theory and Aberration . . . . . . . . . . . . . 18

2.2 .4 Diffraction. . . . . . . . . . . . . . . . . . . . . 20

2.3 Ocular Aberrations . . . . . . . . . . . . . . . . . . . . . . . . 20

2.3.1 Categories of Ocular Aberrations . . . . . . . . . . . . . . 21

2.3.2 Zernike Representation of Aberration . . . . . . . . . . . . . . . . . 21

2.3.3 Wavefront Sensing . . . . . . . . . . . . . . . . . . . 22

2.3.4 Effect of High Order Aberrations . . . . . . . . . . . . . . . . . 24

2.3.5 Chromatic Aberration . . . . . . . . . . . . . . . . 25

2.4 Summary . . . . . . . . . . . . . . . . . . . . . 25

3. METHODOLOGY . . . . . . . . . . . . . . . . . . 27

3.1 Modeling of Image Viewing _ . . . . . . . . . . . . . . . . . 27

3.1 .1 Reconstruction of Ocular Aberration . . . . . . . . . . . . . 27

3.1 .2 Point Spread Function . . . . . . . . . . . . . . . . . . . . . . 28

3.1 .3 Retinal Image Formation as Convolution . . . . . . . . . . . . . . . 30

3.1.4 Modulation Transfer Function . . . . . . . . . . . . . . . . . . . 31

3.1 .5 Limitation of Retinal Image . . . . . . . . . . . . . . . . . . . 32

3.1.6 Viewing Distance and Aberration . . . . . . . . . . . . . . . . . 35

3.1 .7 Computation of PSF . . . . . . . . . . . . . . . . 38

3.1.8 Simulation of What Computer Users View . . . . . . . . . . . . 43

3.2 Image Precompensation of Aberration . . . . . . . . . . . . . . 47

3.2 .1 Comparison with Image Restoration . . . . . . . . . . . . . . . . 48

3.2 .2 Inverse Blurring Filtering . . . . . . . . . . . . . . . . . . . . 49

3.2 .3 Contrast Loss of Precompensation _. . . . . . . . . . . . . . 51 
3.2.4 Software Simulation . . . . . . . . . . . . . . . . . . . 52

3.2.5 Contrast Improvement by Histogram Clipping . . . . . . . . . . . . . . 54

3.3 Dynamic Image Compensation . . . . . . . . . . . . . . . . . . 58

3.3 .1 Dynamics of Aberration . . . . . . . . . . . . . . . . . . . 59

3.3.2 Effect of Pupil Size on Aberration . . . . . . . . . . . . . . . . 61

3.3 .3 Aberration Resizing . . . . . . . . . . . . . . . . . . . . . 62

3.3.4 Comparison of Measured Aberration and Resized Aberration . . . . . 66

3.3.5 Software Simulation . . . . . . . . . . . . . . . . . . . . . 69

3.3 .6 System Diagram . . . . . . . . . . . . . . . . . . . 70

3.4 Summary . . . . . . . . . . . . . . . . . . . . . . 73

4. ARTIFICIAL EYE EXPERIMENT . . . . . . . . . . . . 75

4.1 Experimental Instruments . . . . . . . . . . . . . . . . . 75

4.2 Experimental Design . . . . . . . . . . . . . . . . . . 76

4.3 Results and Discussion . . . . . . . . . . . . . . . . . 78

4.4 Summary . . . . . . . . . . . . . . . . . . . . . . . . 79

5. HUMAN SUBJECT EXPERIMENT . . . . . . . . . . . . . . . 82

5.1 Experimental Images . . . . . . . . . . . . . . . . . . . . . . . 83

5.2 Subjects . . . . . . . . . . . . . . . . . . . . . . . 84

5.3 Experimental Setup . . . . . . . . . . . . . . . . . . . . 86

5.3 .1 Instruments . . . . . . . . . . . . . . . . . . . . . 86

5.3 .2 User Interfaces . . . . . . . . . . . . . . . . . . . . . . . 87

5.4 Design and Procedures . . . . . . . . . . . . . . . . . . . . . 88

5.4 .1 Image Recognition Test . . . . . . . . . . . . . . . . . . . 90

5.4 .2 Subjective Assessment . . . . . . . . . . . . . . . . . . . . . . . 91

5.5 Results and Discussion . . . . . . . . . . . . . . . . . . . . 91

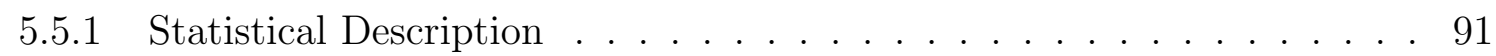

5.5 .2 Results of ANOVA . . . . . . . . . . . . . . . . . . . 92

5.5.3 Results of Subjective Assessments . . . . . . . . . . . . . . . . 98

5.5 .4 Discussion . . . . . . . . . . . . . . . . . . . . . . . . . . . . . . . . . . 99

5.6 Summary . . . . . . . . . . . . . . . . . . . . 101

6. CONCLUSIONS AND FUTURE WORK . . . . . . . . . . . . . . 103

6.1 Concluding Remarks . . . . . . . . . . . . . . . . . . . . 103

6.1 .1 Modeling of Computer User's Retinal Image . . . . . . . . . . . . . . 104

6.1 .2 Dynamic Precompensation . . . . . . . . . . . . . . . . . . . . . . 104

6.1.3 Prototype of Application . . . . . . . . . . . . . . . . . . 105

6.2 Limitations . . . . . . . . . . . . . . . . . . . . . 106

6.3 Future Directions . . . . . . . . . . . . . . . . . . . 107

BIBLIOGRAPHY . . . . . . . . . . . . . . . . . . 109 


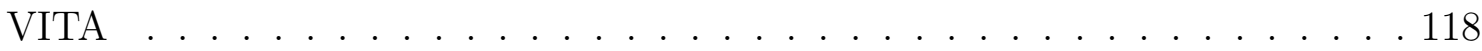




\section{LIST OF FIGURES}

FIGURE

PAGE

2.1 Anatomical side view of the human eye. . . . . . . . . . . . . . . 11

2.2 Angular relationship when light passes from one medium to another denser medium. Reflection angle equals to the incidence angle by the law of reflection. According to the Snell's law, the refraction angle is smaller than the incidence angle due to the larger refractive index. . . . . . . . . . . . . . . . . . 15

2.3 Schematic diagrams illustrating the emmetropic, myopic, hyperopic and astigmatic eyes. . . . . . . . . . . . . . . . . 16

2.4 Illustration of wavefront aberration, which is defined as the difference between the aberrated wavefront and the ideal wavefront. . . . . . 18

2.5 Wavefront propagation based on Huygens's theory. . . . . . . . . . . . . 19

2.6 First six orders of Zernike pyramid of aberrations in which piston, tip and tilt are excluded. Wavefront aberration can be decomposed into a set of Zernike modes [42] . . . . . . . . . . . . . . . . 22

2.7 Principle of wavefront sensing with Hartmann-Shack sensor. . . . . . . . 23

3.1 Examples of wavefront aberration function and normalized PSF corresponding to (a) $-1 \mu m$ of defocus aberration, (b) $-1 \mu m$ of astigmatism aberration, (c) $-1 \mu m$ of coma aberration and (d) a real eye with -4 Diopter of spherical error and $4.5 \mathrm{~mm}$ pupil diameter. . . . . 29

3.2 Retinal image formation as (a) wavefront propagation and (b) convolution process with the Point Spread Function of the eye. . . . . . . . 31

3.3 MTFs corresponding to (a) $-1 \mu m$ of defocus aberration, (b) $-1 \mu m$ of astigmatism aberration, (c) $-1 \mu \mathrm{m}$ of coma aberration and (d) a real eye with -4 Diopter of spherical error and $4.5 \mathrm{~mm}$ pupil diameter. 32

3.4 Airy disk pattern in (a) gray scale image and (b) cross section. The angular radius of the first diffraction ring is related to the diameter of the aperture. . . . . . . . . . . . . . 33

3.5 Rayleigh criterion that limits the resolution of imaging systems. . . . . . 34

3.6 MTF curves of aberration-free eyes with pupil diameter of $3 \mathrm{~mm}, 5 \mathrm{~mm}$ and $7 \mathrm{~mm}$. The cutoff frequency of the MTF increases when the pupil size becomes larger. . . . . . . . . . . . . . . . .

3.7 Geometry demonstrating the wavefront deviation between the plane wavefront originated from an infinite distance and spherical wavefront originated from a finite distance. . . . . . . . . . . . . . 36 
3.8 Comparison of ocular aberrations and normalized PSFs (a) without viewing distance adjustment, (b) with $0.3 \mathrm{~m}$ viewing distance adjustment, (c) with $0.5 \mathrm{~m}$ viewing distance adjustment and (d) with $1.0 \mathrm{~m}$ viewing distance adjustment. The original ocular aberration is measured from an eye with -4.5 D Spherical error and -0.6 D Cylindrical error with $147^{\circ}$ Axis. The pupil diameter of the eye is 5.4 mm. . . . . . . . . . . . . . . . . . .

3.9 Geometry demonstrating the field of view of the eye when viewing the displayed image. . . . . . . . . . . . . . . . . 44 4

3.10 Calibration of the PSF before the convolution. The field of view of the PSF matrix is enlarged by the zero padding operation and reduced by the frame cut operation. . . . . . . . . . . . . . . .

3.11 Example of PSF calibration in which the FOV of the PSF is increased from (a) 100 arcminute to (b) 200 arcminute, in both horizontal and vertical dimensions. . . . . . . . . . . . . . . . . .

3.12 PSFs of an aberrated eye with -5.8 D Spherical error and -1.8 D Cylindrical error with $2^{\circ}$ Axis at viewing distance of (a) $0.3 \mathrm{~m}$, (b) 0.5 $\mathrm{m}$ and (c) $0.8 \mathrm{~m}$, respectively. The PSFs here have been calibrated according to the the viewing distance and the physical size of the displayed image. . . . . . . . . . . . . . . .

3.13 Simulation of retinal images viewed by an aberrated eye with $-5.8 \mathrm{D}$ Spherical error and -1.8 D Cylindrical error with $2^{\circ}$ Axis: (a) Original displayed images, (b) Simulated images viewed at distance of $0.3 \mathrm{~m}$, (c) Simulated images viewed at distance of $0.5 \mathrm{~m}$ and (d) Simulated images viewed at distance of $0.8 \mathrm{~m}$. . . . . . . . . . . . . .

3.14 Schematic of the imaging process with precompensation. The original image is compensated to counteract the image blurring caused by

3.15 Simulation results of image precompensation based on an aberrated eye with -5.8 D Spherical error and -1.8 D Cylindrical error with $2^{\circ}$ Axis: (a) original displayed images, (b) retinal images viewed without precompensation, (c) precompensated images and (d) retinal images viewed with precompensation. . . . . . . . . . . . . . . .

3.16 Histograms of the (a) original images, (b) precompensated images and (c) simulated retinal images.

3.17 (a) Precompensated images and (b) simulated retinal images with contrast enhanced by the histogram side-clipping. . . . . . . . . . . . . 57

3.18 Histograms of the (a) precompensated images and (b) simulated retinal images, after the histogram side-clipping method is applied to expand the histograms of the precompensated images. . . . . . . . . . . 
3.19 Comparison of measured ocular aberrations when the pupil diameter is (a) $4.5 \mathrm{~mm}$, (b) $6.0 \mathrm{~mm}$ and (c) $6.8 \mathrm{~mm}$, respectively. The ocular aberrations are measured from an eye with -8.6 D Spherical error and -0.6 D Cylindrical error with $85^{\circ}$ Axis. The PSFs with three different pupil sizes are also shown in the right column. . . . . . . . . 60

3.20 Overall wavefront RMS error and high order RMS error when the pupil diameter is $4.5 \mathrm{~mm}, 6.0 \mathrm{~mm}$ and $6.8 \mathrm{~mm}$, respectively. . . . . . . . . 62

3.21 Example of ocular aberration resizing: (a) original ocular aberration, (b) resized ocular aberration. . . . . . . . . . . . . . . 67

3.22 Comparison of resized aberration (RA) and measured aberration (MA) with the same $4.5 \mathrm{~mm}$ pupil diameter: (a) measured aberration, (b) resized aberration from $6.8 \mathrm{~mm}$ pupil diameter, (c) resized aberration from $6.0 \mathrm{~mm}$ pupil diameter, (d) error between the measured aberration and the resized aberration from $6.8 \mathrm{~mm}$ pupil diameter and (e) error between the measured aberration and the resized aberration from $6.0 \mathrm{~mm}$ pupil diameter. . . . . . . . . . . . . . . 68

3.23 Simulated retinal images when the precompensated images generated based on the aberration with $4.0 \mathrm{~mm}$ pupil diameter are viewed by the same eye with constricted pupil diameter of (a) $3.2 \mathrm{~mm}$, (b) 3.5 $\mathrm{mm}$ and (c) $3.8 \mathrm{~mm}$, respectively. . . . . . . . . . . . . . . . . . . 70

3.24 Simulated results when the precompensated images are updated according to the resized aberration with the matched pupil diameters at the time of viewing: (a) updated precompensated images and (b) corresponding retinal images. . . . . . . . . . . . . . . 71

3.25 Schematic diagram of the dynamic image precompensation system. . . . 72

4.1 "Artificial eye" used in the experiment. . . . . . . . . . . . . . . 76

4.2 Two $256 \times 256$ test images used in the artificial eye experiment. . . . . . 76

4.3 Wavefront aberration and calibrated PSF of the camera with $6.2 \mathrm{~mm}$ aperture diameter. . . . . . . . . . . . . . . . . 77

4.4 Images captured when the camera was (a) in focus and (b) defocused with -5 D spherical error. . . . . . . . . . . . . 78

4.5 Precompensated images generated according to the camera's wavefront aberration with $6.2 \mathrm{~mm}$ aperture diameter. . . . . . . . . . 79 
4.6 Results of the artificial eye experiment: (a) images captured when the precompensated images were viewed by the aberrated camera with $6.2 \mathrm{~mm}$ aperture diameter, (b)images captured when the precompensated images were viewed by the aberrated camera with $4.0 \mathrm{~mm}$ aperture diameter, (c) images captured when the images with updated precompensation were viewed by the aberrated camera with $4.0 \mathrm{~mm}$ aperture diameter. . . . . . . . . . . . . . . . . .

5.1 Test images used in the recognition tests: (a) eight icons that indicate Copy, Document, Folder, Email, Picture, Printer, Save and Delete, respectively, from left to right and up to bottom, (b) eight example letters (A, B, C, D, W, X, Y, Z) with Arial font. In each test condition, only 8 letters were randomly selected from the alphabet and presented. . . . . . . . . . . . . . . 83

5.2 Complete Ophthalmic Analysis System (COAS-HD, Wavefront Sciences, Inc) that was used to measure the initial ocular aberration data of the subjects. . . . . . . . . . . . . . 86

5.3 Tobii T60 eye-tracking system that was used to monitor the real-time pupil sizes of the subjects during the tests. . . . . . . . . . 87

5.4 Configuration interface at the beginning of the image recognition test. . 88

5.5 Test interface in the image recognition test. . . . . . . . . . . . . 89

5.6 Bar graph of average correct recognitions under different conditions, in which the recognition performances with NPC, SPC and DPC are compared in parallel. p.

5.7 Problems that most impeded recognition of the test images with dynamic precompensation. 11 subjects reported low contrast. 5 subjects reported ringing artifacts and other 4 subjects reported other factors. . . . . . . . . . . . . . . 99 


\section{LIST OF TABLES}

TABLE

PAGE

5.1 Prescription of the subjects and pupil diameters when the ocular aberrations were measured. . . . . . . . . . . . . 85

5.2 Questionnaire conducted after the completion of the image recognition test. . . . . . . . . . . . . . . 92

5.3 Twelve different conditions in the image recognition tests. . . . . . . . . 93

5.4 Descriptive Statistics of the image recognition tests under different con-

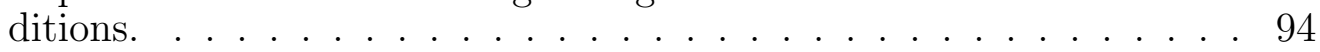

5.5 Results of Mauchly's sphericity test to the experimental data. . . . . . . 95

5.6 Results of within subjects effects test. The main effects of factors with significant value smaller than 0.05 are considered as significant. . . . 95

5.7 Pairwise comparisons between three precompensation methods. The mean difference of two methods is significant at the 0.05 level. . . . . 97 


\section{CHAPTER 1 \\ INTRODUCTION}

\section{$1.1 \quad$ Problem Statement}

With the progress of computer technology and computer software, computers become more and more prevalent in all aspects of people's daily life. Traditionally, computers work in a passive and rigid way to receive the input and commands from humans. The major goal of human computer interaction design is providing interfaces or platforms to make computer operations more intuitive and easier to learn and use. While the demand for more efficient and easier-to-use interfaces still exists, the research focus on human computer interaction was shifted to user adaptive interfaces with more intelligence and user personalization $[17,18,36]$. Meanwhile, the relationship between human and computer as well as their roles in the process of interaction have also evolved. While humans used to distort their intuitive and natural interaction patterns to accommodate the rigid working style of computers, now computers are expected to play a more proactive role and make a larger contribution to improve the interaction with humans [79].

Humans interact with computers relying on three major senses: vision, audition and touch [83]. Among them, visual-based interaction is the most efficient way due to the human vision's advantages. Graphical user interface (GUI) is ubiquitously used in computer programs and internet web pages [56]. The GUI represents information to users through images, icons, menus and other visual indicators to facilitate the use of computers. After nearly 30 years of development, GUI has become the standard user interface in computers and other electronic devices such as cell phones and tablets. In most cases, it works effectively and greatly ease the computer operations for users with normal vision. However, the graphical information presented 
in sophisticated designs can not be retrieved by users with visual problems, due to the visual blurring or degradation caused by various aberrations or illness of their eyes. Actually, people with visual impairments may encounter difficulties on the recognition of icons, menus, and other graphical components, which limit their ability to obtain correct information and thus impede the efficiency of their interaction with computers. This is exactly the scenario where the computer is expected to make a larger effort to satisfy its users' needs. In order to overcome or relieve the visual degradation of computer users, graphical elements can be preprocessed be-

fore presenting them on the screens, taking advantage of the computer's numerical capability. Because of the unique visual characteristics of each human eye, this kind of preprocessing needs to be personalized based on custom models.

\subsection{Objective of Study}

The objective of this dissertation is to propose an image-processing-based method to improve the visual performance of computer users with ocular aberrations, through dynamic and real-time preprocessing to the graphical information before display. With the measured aberration data of users' eyes, the images that will be presented on the screen are filtered and compensated in real-time according to their specific ocular aberrations. In practice, the aberrations of the eyes are not static and tend to be altered along with many factors. This brings difficulties when we try to build the accurate compensation model of users at the time of viewing. One critical factor is pupil size since it is very sensitive to illumination conditions. In order to overcome the challenges to aberration modeling caused by pupil size variations, pupil diameters of users will be monitored and recorded to update the compensation model in a dynamic way. 
To assess the effectiveness of this method, the image precompensation method was first verified through an "artificial eye" (a high-resolution camera) experiment, which provides access to the perceived images directly and objectively. The effectiveness of this method was also evaluated through an empirical evaluation with human subjects, in which both quantitative results and subjective assessments from subjects were recorded for analysis.

\subsection{Significance of Study}

People with visual impairment usually have difficulty to use computers and access the internet. It is reported that $70 \%$ of people with visual impairments have never used computers and approximately one fifth of them have no internet access [52]. In order to help the visual impaired individuals to use computers and the internet, it is necessary to aid them in locating the information required to complete various tasks. The need for computer accessibility systems for visual impaired people is receiving more and more attention from different research communities, due to the increasing number of individuals in this group $[10,88]$ and the increasing demands for assistive technologies driven by relevant organizations. According to a report of World Health Orgnization (WHO), over 285 million people are visually impaired worldwide, of whom 39 million are blind and 246 million have varying degrees of visual impairment. It is predicted that number of visually impaired individuals will rise to 200 million by the year 2020. Globally, uncorrected refractive errors are the number one cause of visual impairment [55]. Generally, ocular aberration includes low order aberrations (e.g., myopia, hyperopia) and high order aberrations (e.g., Keratoconus). Myopia affects more than 30.5 million Americans age 40 and older and hyperopia affects 5 to $10 \%$ of Americans 40 and older. By adulthood, it is 
reported that about 25\% Americans are myopic and require some form of optical correction to see clearly beyond an arm's length. Besides, approximately 7 million people in the United States alone have some type of high order refractive aberrations in their eyes [52] and Keratoconus affects one in every 2000 Americans [51].

Traditionally, ocular aberrations are corrected by spectacles or contact lenses. More recently, refractive surgeries (e.g., LASIK) have also become a popular alternative, by reshaping the cornea using a laser [12]. These correction methods work well in most cases. However, for some people with visual impairments (e.g., cataracts, glaucoma, macular degeneration), low vision problems still exist even when spectacles or contact lenses are used. In addition, optical spectacles are generally only used to correct the low order aberrations and are not effective for high order aberrations. In fact, it was not possible to measure the accurate high order aberrations of the human eyes in any practical way until the appearance of the Shack-Hartmann wavefront sensor [47]. After that, studies were performed by using adaptive optics techniques to correct high order aberrations of the human eyes to achieve further visual improvements $[14,48,74]$. The effects of high order aberrations were evaluated through standard vision tests (e.g., visual acuity and contrast sensitivity) and also by recognition of natural images in daily activities (e.g., familiar faces) $[23,77]$. However, owing to the high cost and physical size limitations of the adaptive optics system, instruments based on it are not practical to be used as conventional means for daily activity purposes, at present.

Therefore, it is still meaningful to seek new approaches to address the visual problems of computer users in more flexible and efficient ways. This study is an exploration of a potential approach for improving visual performance of computer users without using spectacles, contacts or other special optical devices, with the intention to develop a more versatile visual-based interaction mechanism in which 
the computer is able to adapt the information it presents based on the users' visual characteristics. As the proposed image precompensation method is based on image processing without any special hardware requirements, it can be implemented on any regular personal computers and display devices. It is also promising for potential use in mobile devices such as smart phones and tablets.

\subsection{Literature Review}

The image compensation method in this study preprocesses the images displayed on the computer screen in advance, based on the visual characteristics of different users, thereby enhancing their visual perception at the time of viewing. Image enhancement techniques have been widely used to improve low quality images in order to make them more suitable to be viewed in different applications [20]. General image enhancement techniques usually seek to optimize certain desired features (e.g., high frequency information, histogram distribution) to improve details or contrast. Under most circumstances, the performance or effects of these methods are assessed with the assumption that the image viewers have normal vision. Moreover, most of the image quality assessment approaches rate the image quality by metrics based on the fidelity or similarity with respect to a reference image which is considered to have "perfect" quality [80].

The simplest and most widely used image quality metrics are the peak signal-tonoise ratio (PSNR) and the mean-squared error (MSE), due to their mathematical convenience and simple calculation [91]. These "objective" assessment metrics fall short of consideration of the image observer's perception characteristics. The practical human perception of quality is not always consistent with the value of these metrics. This problem is particularly pronounced in those application scenarios in 
which the image viewers have visual problems, because the "perfect" reference is not valid anymore and there are no general approaches to generate referential images. Therefore, other image quality assessment models [80,92] and image enhancement methods involving human visual system (HVS) characteristics [34, 40] have been created, in an attempt to incorporate human perceptual factors such as contrast sensitivity function (CSF) and luminance condition. However, it is still not feasible to find a reliable and general objective metric to assess the visual quality of people with visual impairments, since the visual degradation caused by different visual problems varies greatly and can not be encapsulated in one assessing model. For the same reason, image enhancement for visually impaired people should be customized according to their visual characteristics and the corresponding visual quality should be assessed by the subjective perception reported by themselves.

So far, several image enhancement methods have been developed aiming to improve the visual performance of people with visual problems, even though the studies and applications in this area are still relatively quite few. Image magnification is probably the most basic enhancement technique and it is widely used by many visually impaired people. The research on screen magnification is still under development and the magnification techniques keep evolving [5]. One inherent problem of image magnification is that the field of view will be reduced when the image is enlarged. Another common strategy is adding extra high frequency components or edges to highlight shape information and enhance the local contrast of the image. Peli [64] proposed a wideband enhancement method to improve the visual perception quality of patients with age-related macular degeneration (ARMD). In this method, visually relevant edge and bar features of the image are extracted and added to the original image as a contour map. Significant improvement was claimed when moderate wideband enhancement was applied on the original images. Wolffsohn [96] 
integrated high-pass edge detection filters in a set-top box to provide real-time enhancement of television images for visually impaired people. Atabany [3] proposed an image enhancement approach for patients with low vision based on scene simplification. Before detecting edge information and adding them into the original image, Atabany's method first simplifies the image using diffusion filters to remove trivial details.

Exploring approaches beyond generic edge enhancement, Peli [66] proposed an adaptive image enhancement algorithm to allocate lager intensity range of display devices to high frequency components of the image by attenuating low frequency components. Peli [62] also proposed a customized enhancement method based on a pre-emphasis model, which is derived from the ratio of the patient's CSF and the CSF of people with normal vision. In subsequent studies, similar methods were used to improve the text reading [16] and face recognition ability [65] of visually impaired people. Similarly, Lawton $[43,44]$ reported that the reading speed of age-related macular degeneration patients was significantly faster when less visible spatial frequency components were enhanced by customized filters according to the normalized CSF of the patients. Leat [45] compared the performance of various customized enhancement filters and generic enhancement filters and reported that visually impaired people preferred filters with high and medium frequency emphasis, without too much attenuation of lower frequencies. In the above studies, enhancement models were mainly developed based on the patient's CSF, as an approximation to the frequency modulation ability of the patient's eyes. However, this empirical characterization is highly sensitive to measurement parameters and does not account for the dynamic behavior of the human eye.

Tang [82] proposed an enhancement method for JPEG images viewed by low vision viewers, in which the enhancement is integrated in the process of JPEG 
compression. Tang's algorithm enhances the image contrast in the discrete cosine transform domain by adjusting weights of the quantization table in the decoder. Image enhancement techniques for video images were also developed to facilitate watching video, especially television, by low-vision people $[41,63]$.

The studies introduced above mainly aimed to improve the visual performance of people with various visual impairments, but did not address the visual blurring caused by the general aberrations of the eyes. Although ocular aberrations such as myopia and hyperopia are usually not considered as diseases, refractive errors are common reasons causing visual impairments [55]. Moreover, visual impairments are always concomitant with severe ocular aberrations. Alonso [1,2] proposed a software-based method to neutralize the visual degradation, by which the images displayed on the screen were precompensated offline, in advance, based on the ocular aberrations of each specific computer user. This method yielded significant improvement of visual performance. However, the effect of this method was not as large as expected. It was speculated that this is most likely due to the mismatch between the measured ocular aberration that is used to generate the compensation and the ocular aberration at the time of actual viewing, as the optical properties of the human eye are not static but fluctuate over time. The variations of pupil size, primarily caused by the changes of the illumination conditions, can produce considerable impacts on the aberration of the eye. In Alonso's study, the pupils of subjects were pharmacologically dilated by using a combination of tropicamide and phenylephrine before tests, in order to minimize pupil variations. Although this approach lessened the pupil size mismatch problem, it is not an essential solution that could be used in practical applications. Therefore, to further boost the performance of this method and make it more robust under different viewing conditions, the precompensation should be dynamically updated along with the pupil variations, 
instead of being based solely on static aberration data obtained from measurement. Furthermore, in order to make it more feasible to apply image precompensation into practical applications, images with precompensation need to be computed and presented instantly after the user's request, instead of being generated offline, in advance.

\subsection{Dissertation Structure}

The rest of dissertation is organized according to following structure. Chapter 2 introduces the background information of this study, mainly describing the human visual system with geometrical optics and wave optics theory. Ocular aberration and wavefront sensing technology will also be introduced. Chapter 3 presents the modeling of the viewing process of the computer user, in terms of Zernike polynomials, wavefront aberration, Point Spread Function and Modulation Transfer Function. Chapter 3 also demonstrates the precompensation algorithm, wavefront resizing method and the simulation results of the precompensation. In order to improve the contrast of the precompensated image, a histogram clipping method will be introduced. Chapter 4 presents the experimental results of the "artificial eye" test, to evaluate the visual quality of the images with precompensation. The human subject experiment is introduced in Chapter 5, with description of the experimental design and procedures. In addition, Chapter 5 presents the statistical analysis results of the experimental data as well as the subjective assessment from the subjects. Chapter 6 concludes this dissertation and summarizes the contributions and limitations of the study. Finally, Chapter 6 also provides the possible directions for future work. 


\section{CHAPTER 2}

\section{BACKGROUND}

\subsection{Human Vision System}

Human vision is a combination of optical image formation of the external world and neural processing of the light information recorded on the retina. Image formation on the retina is the first step in the visual process. After that, the optical image is converted into a discrete, neural representation by light-sensitive photoreceptors on the retina. This discrete neural image is then processed by a series of complicated resampling operations and finally transmitted to the brain through the optic nerve

to generate the visual perception. Due to the multiple sampling operations and transmissions, the visual information perceived by the brain is always different from the original retinal image formed by the optics of the eye. The neural processing of human vision is far more complicated to be modeled and analyzed in a mathematical way. In this study, we only focus on the modeling and compensation of the optical degradation of the human eye.

The human eye is one of the most complex and sophisticated physiological systems in the human body. Any optical component in the eye has the potential to impair the fidelity of the image formed on the retina and thereby affects the final visual perception. The components of a typical human eye are illustrated in Figure 2.1, as an anatomical side view. The optical system of the eye is mainly composed of the cornea, the iris, and the lens. When light enters into the eye, it will first pass through the cornea, then subsequently through the aqueous humor, the iris, the lens, and the vitreous humor before finally reaching the retina. 


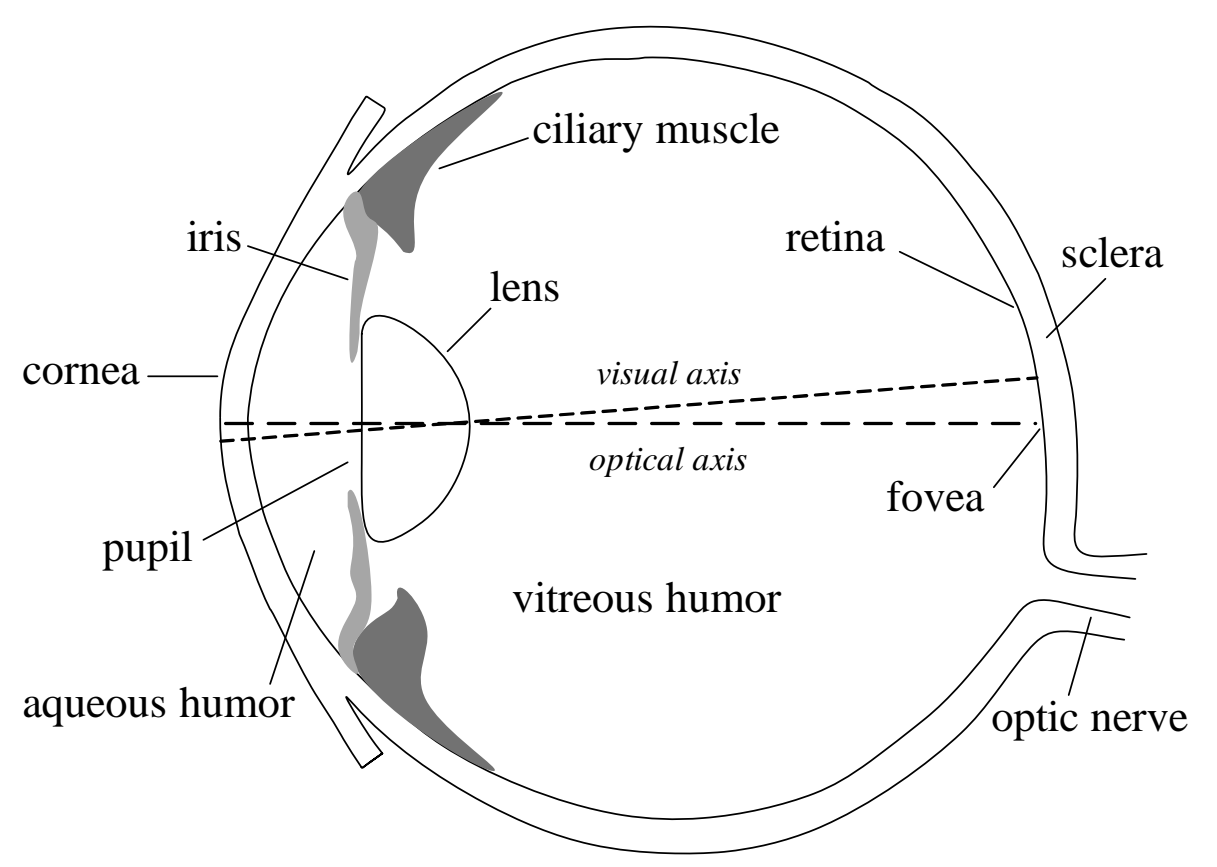

Figure 2.1: Anatomical side view of the human eye.

\subsubsection{Cornea}

The cornea is the transparent front surface of the eye that covers the iris and the pupil. It is an extension of the sclera, which is the white outer shell of the eye. The size of cornea is approximately $12 \mathrm{~mm}$ in diameter and its thickness is approximately 0.5 to $0.6 \mathrm{~mm}$ at the center. The surface of cornea has a radius of curvature of about $7.7 \mathrm{~mm}$ and its total refractive power is roughly 43 Diopters (D) [71]. The cornea accounts for about two-thirds of the refractive power of the eye, thereby contributing most of the ocular aberrations. While the cornea contributes most of the eye's refractive power, its focus length is fixed. The variants of corneal shapes can cause astigmatism and other higher order aberrations $[59,90]$. 


\subsubsection{Lens and Accommodation}

The crystalline is a transparent, biconvex material that located immediately behind the iris and pupil. Combined with the cornea, the lens refracts light that enters into the eye and its refractive power in natural environment is about 20 diopters. The lens is surrounded by a ciliary muscle which can contract and change its shape to adjust the refractive power. Contraction of the ciliary muscle increases the curvature of lens, thereby making the eye focus on near objects. Reversely, relaxing of the ciliary muscle reduces the curvature of lens and allows the eye to focus on distant objects. This ability to change refractive power of lens is called "accommodation", which provides an operating range for focusing that extends from infinity to less than $10 \mathrm{~cm}$ for young people. The accommodation ability of the lens decreases with age and eventually disappears around 55 years of age when the lens become completely inflexible [19].

\subsubsection{Iris and Pupil Response}

The iris is a circular diaphragm lying between the cornea and the lens, controlling the size of pupil and thus the entry of light into the eye. Its function is similar to the shutter in a camera. The pupil is a variable opening located at the center of the iris and determines the amount of light that passes through to the retina, with the function similar to the camera's aperture.

The pupil of the human eye can constrict to $1.5 \mathrm{~mm}$ in diameter and dilate to about 8 to $9 \mathrm{~mm}$. Generally, the pupil has a rapid reaction (constriction or dealation) to stimuli, with the response peaking in 0.5 to $1.0 \mathrm{~s}$. The constriction and dilation of pupil are accomplished by two opposing sets of muscles in the iris, the sphincter pupillae and dilator pupillae, which are governed by the sympathetic and 
parasympathetic divisions of the autonomic nervous system respectively [33]. The constriction of pupil is caused by excitation of the circular pupillary constriction muscle, which is innervated by fibers of the sphincter pupillae from the parasympathetic division of the autonomic nervous system. The sympathetic division, mediated by posterior hypothalamic nuclei, causes the dilation of pupil through the contraction of dilator muscle, which is innervated by sympathetic nerves from the superior cervical ganglion $[6,81]$.

The pupil is very sensitive to the intensity of the light entering the eye. High intensity light causes the pupil to become smaller, thus allowing less light to enter into the eye. Whereas, low intensity light causes the pupil to become larger, thus allowing more light to enter into the eye. The pupil response to light intensity is realized by the physiological mechanism called pupillary light reflex (PLR) [39], which causes the pupil to change its diameter inversely to the amount of light reaching the retina.

The pupil size of the human eye is not constant, but fluctuating even under a constant ambient light condition. During the last decades, it has been reported that the pupil diameter is also dependent on affective stimulus and mental activities [24]. This can be explained by the fact that the nervous system transmits neuronal signals to the sphincter pupillae and dilator pupillae, which will cause the pupil to dilate or to contract. Studies $[29,35,50]$ have indicated the pupil size is correlated with mental workload. It was also found that pupil size variation was related to cognitive information processing $[22,69]$. Partala [60] found that pupil size is significantly larger under auditory emotional stimuli than under neutral stimuli. More recently, Ren [72] found the pupil diameter signal can be used as an indication of the stress state of the computer user. 


\subsubsection{Photoreceptors}

The image formed on the retina is a continuous distribution of the light intensity recorded in terms of the external objects. Before further neural processing, the light that passes through the pupil is sampled by a set of light-sensitive photoreceptors called rods and cones on the surface of retina. Therefore, the visual resolution of the human eye is limited by the density of photoreceptors. In the human eye, the highest density of photoreceptors is located on the center of the fovea, where photoreceptors are exclusively cones and provide a cutoff frequency of about $120 \mathrm{cyc} /$ degree [94,95]. This means that the maximum spatial frequency that the eye can detect without aliasing is around $60 \mathrm{cyc} /$ degree, based on the Nyquist sampling theorem.

\subsection{Geometrical and Wave Optics}

In spite of the complicated structure of the human eye, the retinal image formation is an optical imaging process, which essentially has no difference with other optical systems like a camera. In this section, we will introduce some basic optics knowledge, that will help us to understand how image is formed and distributed on the retina.

\subsubsection{Reflection and Refraction}

In geometric optics, light rays are a simplified abstraction used to model the propagation of light through an optical system. When light passes through the boundary of two dissimilar media, it undergoes reflection and refraction, as shown in Figure 2.2. Reflection is the direction change of light so that the wavefront bounces back to the medium that it is from. The reflection law says that for specular reflection 


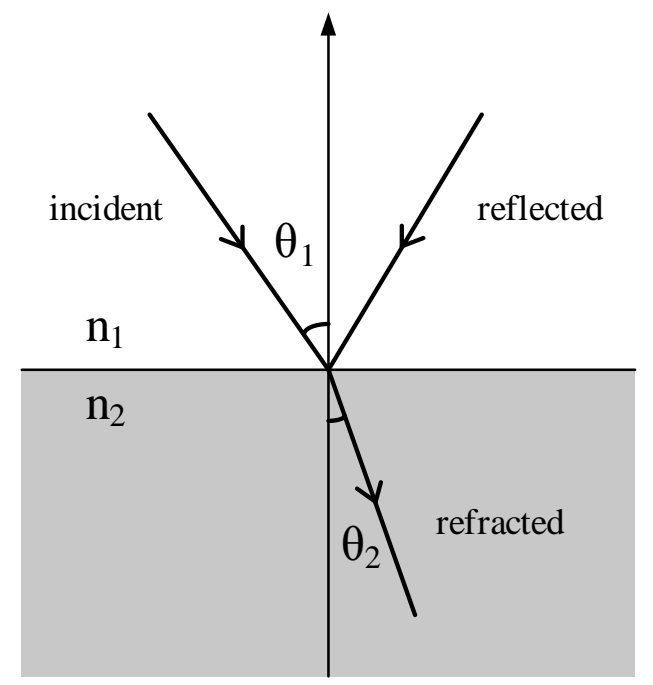

Figure 2.2: Angular relationship when light passes from one medium to another denser medium. Reflection angle equals to the incidence angle by the law of reflection. According to the Snell's law, the refraction angle is smaller than the incidence angle due to the larger refractive index.

the angle at which the light is incident on the surface equals to the angle at which it is reflected.

Refraction is the phenomenon that takes place when light passes through the interface of two different media and the light direction is changed suddenly due to the different refractive indices of the meida. The refractive index of a medium is defined as

$$
n=\frac{c}{v},
$$

where $c$ is the speed of light in vacuum and $v$ is the phase velocity in the corresponding medium. The refractive index of air at $0{ }^{\circ} \mathrm{C}$ is about 1.0003 . The refractive index of cornea is not uniform, around 1.37 [61].

Based on Snell's law [38], incidence angle $\theta_{1}$ and refraction angle $\theta_{2}$ are related by,

$$
n_{1} \sin \theta_{1}=n_{2} \sin \theta_{2}
$$




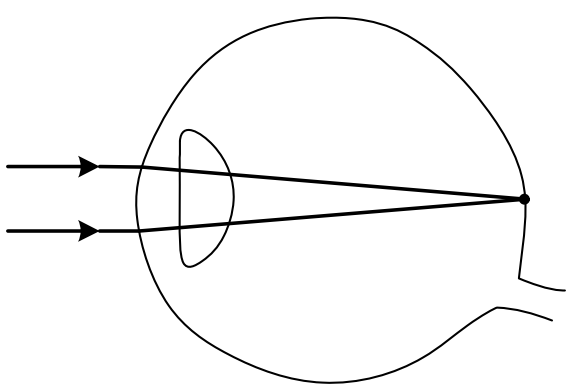

Emmetropia

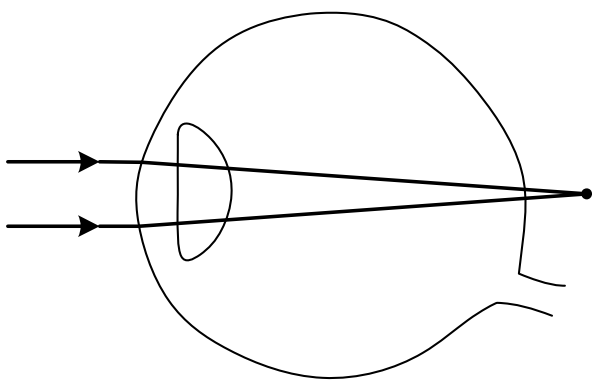

Hyperopia

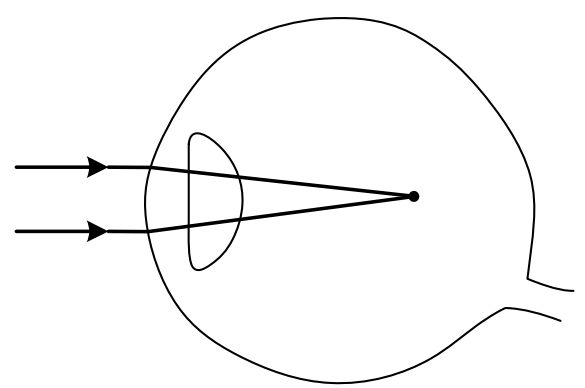

Myopia

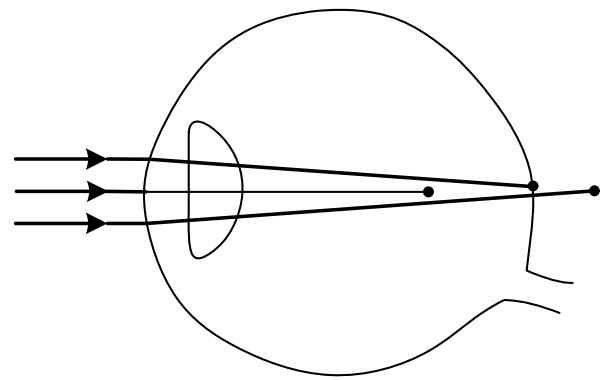

Astigmatism

Figure 2.3: Schematic diagrams illustrating the emmetropic, myopic, hyperopic and astigmatic eyes.

where $n_{1}$ and $n_{2}$ are the refractive indices of two separate media respectively. This relationship is due to the changing of phase velocities of light in different media. The denser the medium, the slower light transmits in it. Snell's law also states that the ratio of phase velocities in the two media is equivalent to the opposite ratio of the indices of refraction:

$$
\frac{n_{1}}{n_{2}}=\frac{v_{2}}{v_{1}}
$$

where $v_{1}$ and $v_{2}$ are the velocities of light in the two media.

\subsubsection{Paraxial Theory and Refractive Error}

The imaging process can be further simplified by the use of paraxial approximation. The paraxial approximation is effective when light rays are traveling close to the optical axis, at small angles with the optical axis, as $\sin \theta$ approximately equals to $\theta$. 
Applying it, the Snell's law reduces to a simpler relationship between the incidence angle and the refraction angle:

$$
n_{1} \theta_{1}=n_{2} \theta_{2}
$$

The paraxial model and theory can be used to predict the location and magnification of an image effectively.

As an optical system, the human eye focuses the rays of light from a distant point entering the eye into a single image point on the retina. When light enters into the eye from the air, it is refracted by the cornea and the lens. Refractive errors occur if the eye can not refract the light properly and fails to focus the light directly on the retina, as shown in Figure 2.3. Refractive errors cause the image distributed on the retina to be blurred. An eye that has no refractive error when viewing distant objects is called emmetropic eye.

Types of refractive error include myopia, hyperopia and astigmatism. Myopia and hyperopia are caused by the optical power being either too large or too small, which is also called spherical error. Myopia occurs when the refractive power of the eye is too large and the light focuses in front of the retina. It is usually caused by a cornea with too much curvature. Hyperopia occurs when the refractive power of the eye is too small and the light focuses behind the retina. It is usually caused by a cornea with not enough curvature. Astigmatism is caused by the cylindrical error of the eye. Ideally, the shape of cornea is symmetrical, and light entering from all angles is refracted with the same amount. In reality, the cornea and the lens sometimes become asymmetrical with irregular shape, causing the light entering the eye to focus differently in two meridians. 


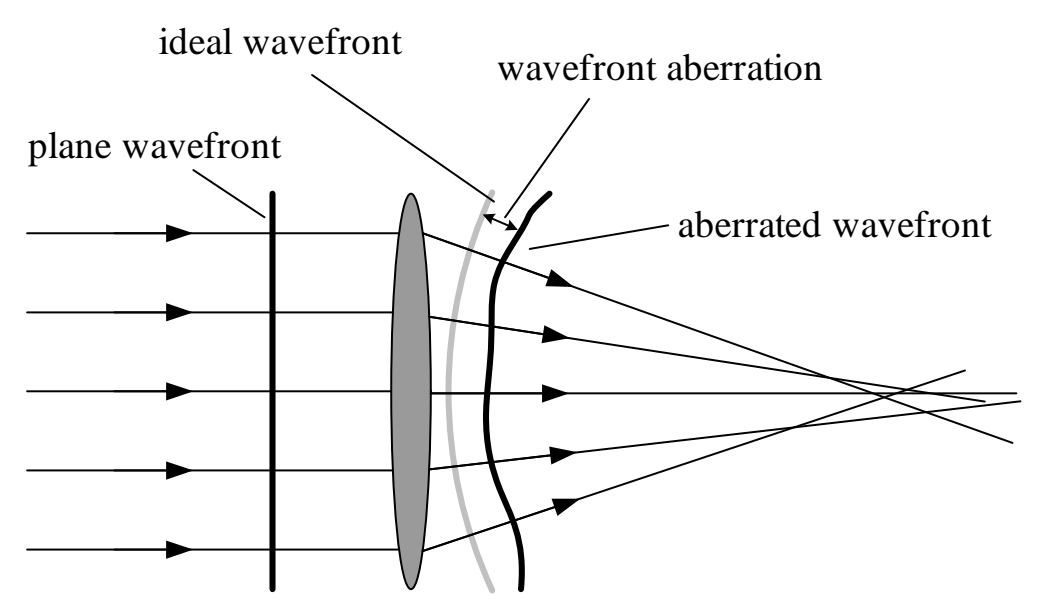

Figure 2.4: Illustration of wavefront aberration, which is defined as the difference between the aberrated wavefront and the ideal wavefront.

\subsubsection{Wavefront Theory and Aberration}

The human eye suffers from three types of imperfections (aberration, diffraction and scattering), which are the sources of the blurring of the image formed on the retina. In general, refractive errors can be described and modeled by geometrical optics methods conveniently. However, beside refractive errors, there are other irregular errors exist in the human eye, which are difficult to model with geometrical optics. In addition, geometrical optics can not be used to explain other optical phenomenons such as interference and diffraction.

From a geometrical perspective, the wavefront of light is assumed to have a spherical shape characterized by a center of curvature and a radius of curvature and the light rays are always perpendicular to the light wavefront. In wave optics, light is described as a propagating wave. A wavefront is an imaginary surface over which an optical disturbance has a constant phase. When light emerges from a point source, the wavefront appears as a spherical shape. As the wavefront moves further and further from the source, the surface of the wavefront will get flatter and flatter, finally becoming plane wave at a great distance. 


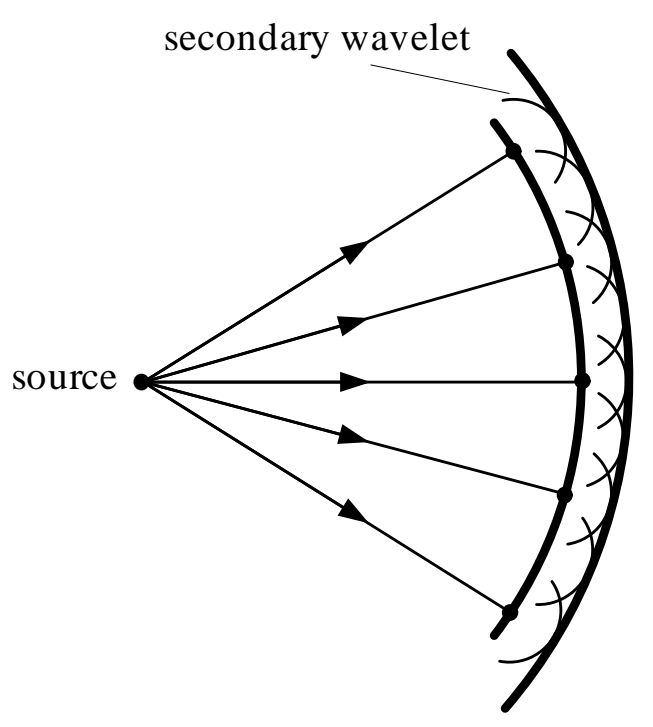

Figure 2.5: Wavefront propagation based on Huygens's theory.

According to the wave theory of image formation, the perfect image of a point object is formed by a collapsing hemispherical wavefront with center of curvature located in the image plane [84]. When the wavefront is interrupted by media of a different index, the emerging wavefront shape is often deviated and does not converge to a point. The deviation of the wavefront occurs if the surface of the medium is not smooth or the refraction index of the medium is not constant [42]. Therefore, the wavefront aberration is defined as the difference between the actual aberrated wavefront and the perfect or intended wavefront, as shown in Figure 2.4. In reality, the human eye is not a perfect optical system. The imperfection of the human eye can be described numerically through the wavefront aberration. The imperfection pattern of each human eye is usually unique, thus the wavefront aberrations of different eyes are different and degrade the retinal image formed in different patterns. 


\subsubsection{Diffraction}

Even though the optical system of an eye functions perfectly without any aberrations, the image formed on the retina will still have distortion due to the diffraction phenomenon in the eye. The diffraction occurs when a wave of light encounters small obstacles during propagation. Based on the Huygens principle, any wavefront can be considered to be composed by an infinite number of point sources propagating light and every point source on the wavefront is the center of a new, secondary, disturbance which emanates spherical wavelets in the direction of propagation. Thus, the wavefront at any later instant would be the envelope of these secondary wavelets [84]. This principle is illustrated in Figure 2.5. When a wavefront encounters an aperture

(e.g., pupil), the shape of the wavefront is changed because of the superposition and interference of the waves from the point sources that pass through the aperture. The blurring cause by diffraction is unavoidable and usually ignored when the pupil size is large. However, when the pupil size becomes very small, the diffraction is an important factor that affects image quality in the human eye.

\subsection{Ocular Aberrations}

Like in other optical systems, the image blurring on the retina is mainly caused by its optical aberrations, which are called ocular aberrations. Ocular aberrations consist of the low order aberrations and high order aberrations, which are also called spherocylindrical errors and irregular errors, respectively. Every human eye has unique aberrations with personalized patterns. This uniqueness occurs not only in the eyes of different people, but also in each eye of the same person. Even when a person's eyes have equal prescription of glasses or contact lenses, their actual ocular aberrations are different. 


\subsubsection{Categories of Ocular Aberrations}

Ocular aberrations include low order aberrations and high order aberrations. Low order aberrations of the human eye mainly indicate myopia, hyperopia, and astigmatism, which are second order aberrations. Other non-visually significant aberrations such as piston, tip and tilt, also belong to the low order aberrations. In general, low order aberrations contribute most of the aberration of the human eye and account for most of the image degradation on the retina. Therefore, traditional vision correction focuses on the correction of low order aberrations, usually by spectacles and contact lenses.

High order aberrations refer to irregular wavefront errors beyond defocus and astigmatism, of which spherical aberration, coma and trefoil are most concerned. High order aberrations tend to increase with pupil size [47]. People with large amount of high order aberrations can have severe visual problems such as halos and glares [12], particularly in low illumination conditions.

\subsubsection{Zernike Representation of Aberration}

The wavefront aberration can be decomposed into a set of basis functions, in a similar way as an image is decomposed into spatial frequency components. The most widely used basis functions to describe ocular aberrations are Zernike polynomials. Figure 2.6 shows the first six orders of the Zernike pyramid of aberrations (piston, tip and tilt are excluded). The Zernike polynomials have desirable mathematical properties due to their orthonormality. In addition, contrary to other basis functions, each Zernike polynomial provides minimum wavefront variance and high order polynomials can be balanced by low order polynomials [12]. 


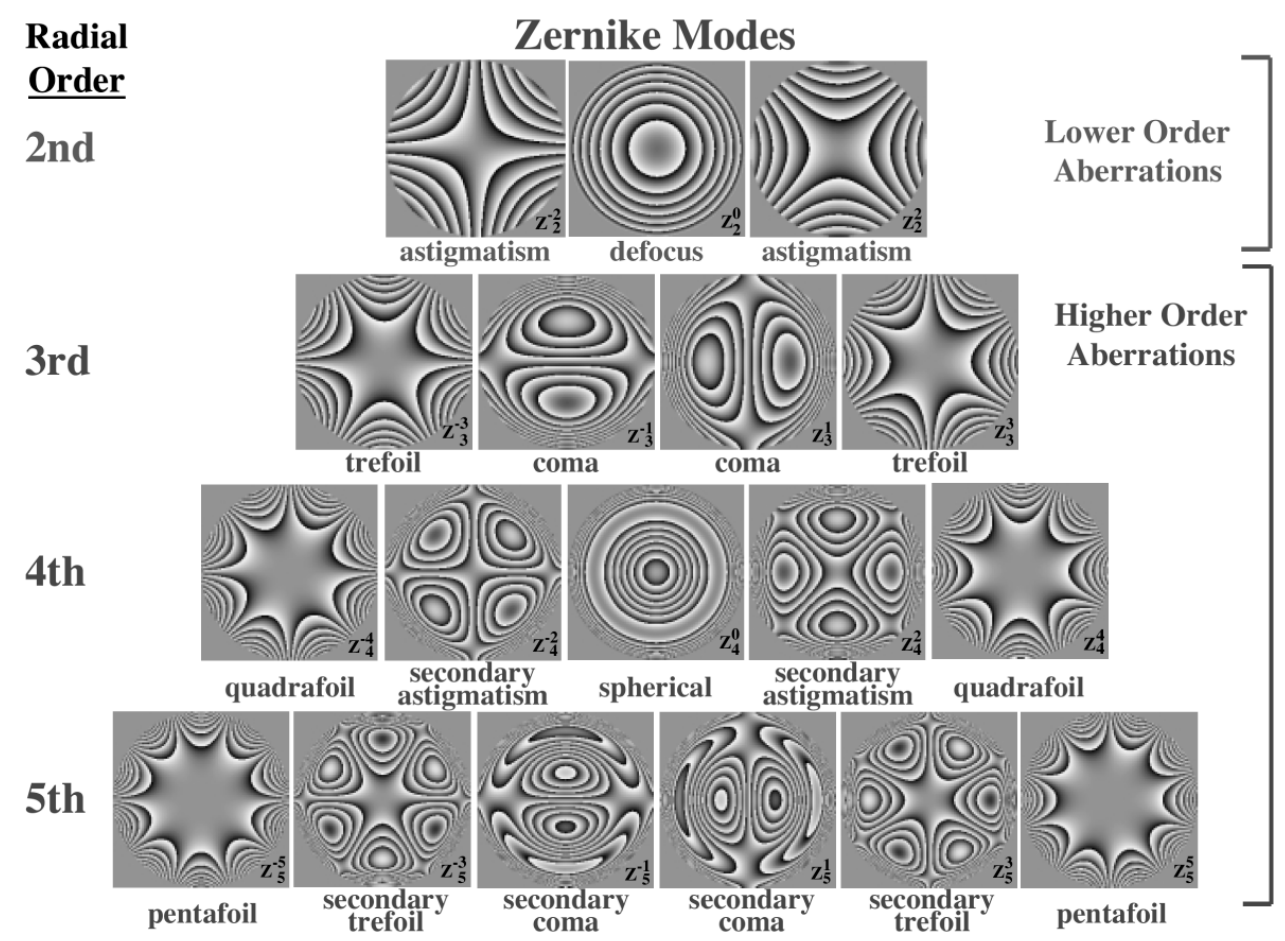

Figure 2.6: First six orders of Zernike pyramid of aberrations in which piston, tip and tilt are excluded. Wavefront aberration can be decomposed into a set of Zernike modes [42].

The root mean squared (RMS) wavefront error of each Zernike mode is given by its coefficient and usually expressed in microns. Generally, the defocus mode is expected to have the largest aberration, followed by two zernike modes of astigmatism. For the human eyes, the magnitude of aberrations tend to decrease with increasing order [26]. It was also found that most Zernike modes are relatively uncorrelated with each other across the population and many aberrations in the left eye are significantly correlated with their counterparts in the right eye [70].

\subsubsection{Wavefront Sensing}

The wavefront sensing technique [68], which is used to measure the wavefront aberration of the eye, is the foundation of customized vision correction. The early 


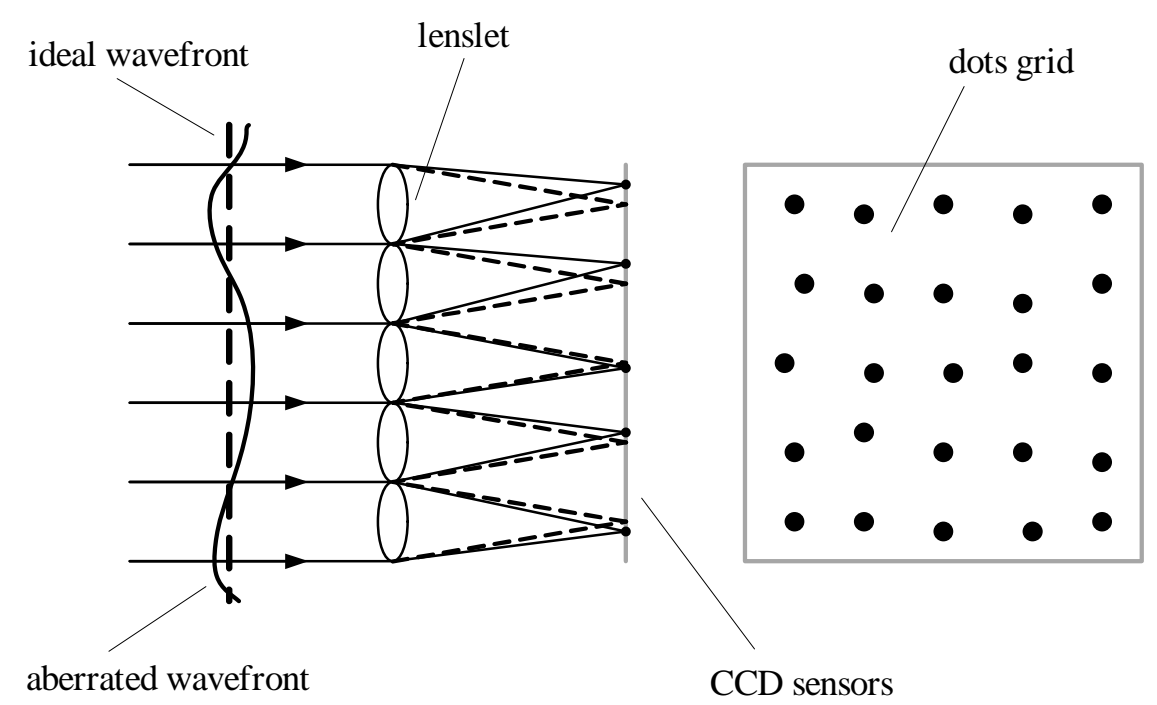

Figure 2.7: Principle of wavefront sensing with Hartmann-Shack sensor.

wavefront sensing techniques are primarily based on the spatially resolved refractometer [93] or ray tracing [58,89]. In 1994, Liang [46] proposed a new wavefront sensing technique based on Hartmann-Shack sensor. The Hartmann-Shack sensor was further improved by Liang and Williams [47] to provide a more complete description of the aberrations of the eyes, measuring up to 10 order aberrations, corresponding to 65 Zernike modes.

The principle of the Hartmann-Shack wavefront sensor is shown in Figure 2.7. During the measurement of Hartmann-Shack sensor, a laser beam is directed to the eye and the light is focused and distributed by the eye's optical system on the retina. The reflected light from the retina is then spatially sampled by an array of small lenses (lenslets) arranged in two dimensions, into a grid of light spots on the focal plane, which are recorded by a CCD sensor placed in the plane. For an eye free from aberration, the light reflected back will be a plane wave and the light spots formed on the CCD sensor will have no displacement. For an aberrated eye, the reflected light will be distorted due to the aberration and the spots formed will be displaced. The displacement of each light spot is determined by the slope of the wavefront at 
the position of the corresponding lenslet. Therefore, the wavefront aberration of the eye being measured can be integrated and calculated by comparing the grid of displaced dots with the reference grid. The Hartmann-Shack sensor provides a fast, objective and accurate way to measure the wavefront aberration of the eye.

\subsubsection{Effect of High Order Aberrations}

Compared with low order aberrations, the magnitudes of high order aberrations are relatively small and have no noticeable effect on the image quality for the normal people, with small pupil sizes. However, high order aberrations can have a significant impact on the image blurring once the pupil size becomes large. In general, correction of high order aberrations of the human eye has two motivations. First, high order aberrations are corrected for additional vision improvement that can not be obtained by the correction of low order aberrations only. Second, correction of high order aberrations is necessary to improve the visual performance of people with high order visual problems such as keratoconus and other irregular corneal abnormalities.

Currently, the correction of high order aberrations has been implemented with adaptive optics systems [97], customized laser refractive surgery [49] and customized contact lenses [76], in which the effects of high order correction were evaluated by the measurement of visual acuity or CSF. The study by Guirao [23] indicated that the visual benefit varies greatly among eyes with high order correction, based on the evaluation of the performance of 109 normal subjects and 4 keratoconic patients. While some eyes show significant visual benefit, other eyes have almost no benefit from the correction of high order aberrations. It was also indicated that the benefit for keratoconic patients is much larger than for the subjects with normal eyes. Thus, 
compared with the additional visual improvement for the people with normal vision, correction of high order aberration seems more valuable for the patients suffering from high order visual problems at the current stage.

\subsubsection{Chromatic Aberration}

Chromatic aberration is used to describe the differences between wavefront aberrations for light with different wavelengths. The chromatic aberration arises because of chromatic dispersion, which is caused by the refraction index variation with wavelength of light. If a medium has higher refraction index, its focal power will be larger, and vice versa. Chromatic dispersion causes the focus, size, and position of the retinal image to vary with the wavelength [84]. Under normal viewing conditions, the human eye is usually exposed to white light, which contains entire spectrum of visi-

ble light. Most of the brightness in a broad spectral object comes from wavelengths near the peak of the spectral luminosity function $(555 \mathrm{~nm})$, over which the chromatic difference of refraction is within $\pm 0.25 D[85,86]$. In this dissertation, we will ignore the effect of chromatic aberration and focus on monochromatic wavefront aberrations and image formation, as the images we use for precompensation will also be monochrome.

\subsection{Summary}

In this chapter, we introduced some background information related to the image precompensation study in this dissertation, including knowledge of the human vision system, basic geometrical optics and wave optics theory. As an optical system, the human eye behaves like a camera and is composed of several major optical components: cornea, lens, iris and pupil. Geometrical optics is effective to be used 
to analyze refractive errors such as myopia and hyperopia. The concept of wavefront and wavefront aberration were introduced. Ocular aberrations, including low order aberrations and high order aberrations, were introduced in detail. Ocular aberration can be represented numerically by using Zernike polynomials over a unit circle. In practice, aberrations of the eye are usually measured by wavefront sensors. The Hartmann-Shack sensor provides a fast, objective and accurate way to measure the wavefront aberration of the eye. 


\section{CHAPTER 3}

\section{METHODOLOGY}

\subsection{Modeling of Image Viewing}

The human eye is a complex optical system with aberrations, thus the image formed on the retina will be distorted. The image formed on the retina, also referred to as retinal image, is an abstract concept. When a person views the image displayed on the screen, it can only be perceived by the observer and is inaccessible to others. Thus, in order to assess the visual performance of a computer user, we need to characterize the actual retinal image numerically.

\subsubsection{Reconstruction of Ocular Aberration}

To characterize the retinal image, we first need to know the ocular aberration. In Chapter 2, we have indicated that the ocular aberration can be measured by the Hartmann-Shack wavefront sensor. In an actual wavefront sensing device, usually called wavefront analyzer or aberrometer, the measured aberration is usually reported as a set of Zernike coefficients and a pupil diameter or radius. With these measured data, the ocular wavefront aberration $W(r, \theta)$ with pupil radius $P_{r}$ can be reconstructed by

$$
W(r, \theta)=\sum_{i=0}^{\infty} a_{i} Z_{i}(\rho, \theta),
$$

where $r$ is the physical radial variable, $\rho=r / P_{r}$ is the normalized radial variable from 0 to 1 , and $a_{i}$ is the coefficient for the basis function $Z_{i}(\rho, \theta)$, which represents the corresponding Zernike polynomial in the form of polar coordinate. $i$ is the single index of the Zernike polynomials. 


\subsubsection{Point Spread Function}

When light from a point source enters into the eye, instead of converging to a point, it will be distributed on the retina according to the aberration pattern of the eye. This intensity distribution of the point source is called Point Spread Function (PSF). In other words, the PSF is the image an optical system forms of a point source [42]. To date, the PSF is the most successful approach for modeling the retinal image formation.

In Fourier optics [21], the amplitude distribution of the light passing through an eye with no aberration can be described by the Fraunhofer diffraction pattern of the exit pupil. The diffraction pattern and pupil function can be further related by the Fourier transform if the transmission amplitude over the pupil is constant. For an aberrated eye, the effect of aberrations is embodied by the phase shift in the generalized pupil function. Thus, the intensity PSF of the eye can be calculated by

$$
P S F\left(r^{\prime}, \theta^{\prime}\right)=|\mathcal{F}\{P(r, \theta)\}|^{2}
$$

The generalized pupil function $P(r, \theta)$ is defined as

$$
P(r, \theta)=A(r, \theta) e^{-i(2 \pi / \lambda) W(r, \theta)}
$$

in which $A(r, \theta)$ is an attenuation function, $W(r, \theta)$ is the aberration function of the eye and $\lambda$ is the wavelength of the monochromatic light entering into the pupil. The attenuation function $A(r, \theta)$ defines the shape, size and attenuation amplitude of light transmission through the aperture of the optical system. For the human eye, the aperture (pupil) shape is circular and the light transmission across the pupil is considered to be uniform, in general. Thus, the attenuation function $A(r, \theta)$ is usually assigned to be one inside the pupil and zero outside. Figure 3.1 show a few examples of wavefront aberration functions and corresponding PSFs. Obviously, the distribution pattern of the PSF becomes broader as the wavefront error 

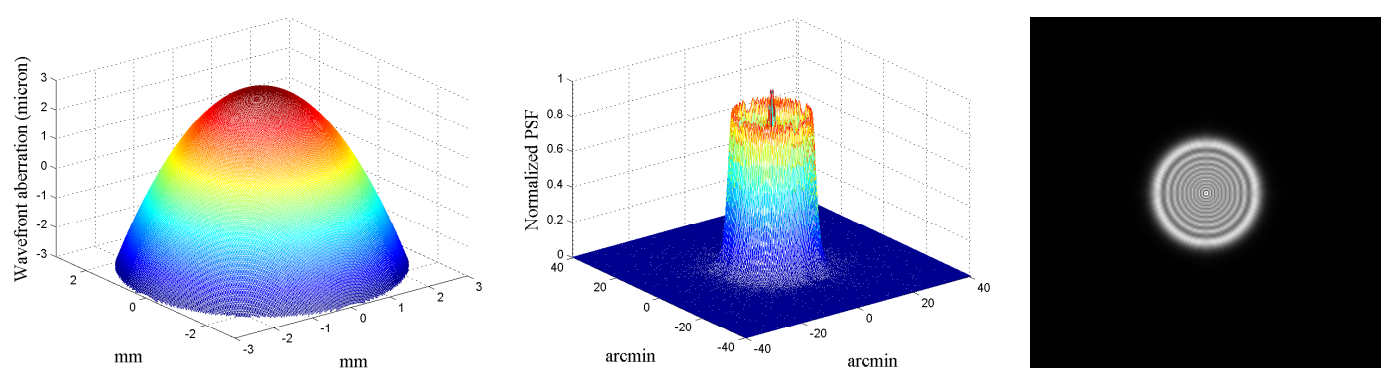

(a)
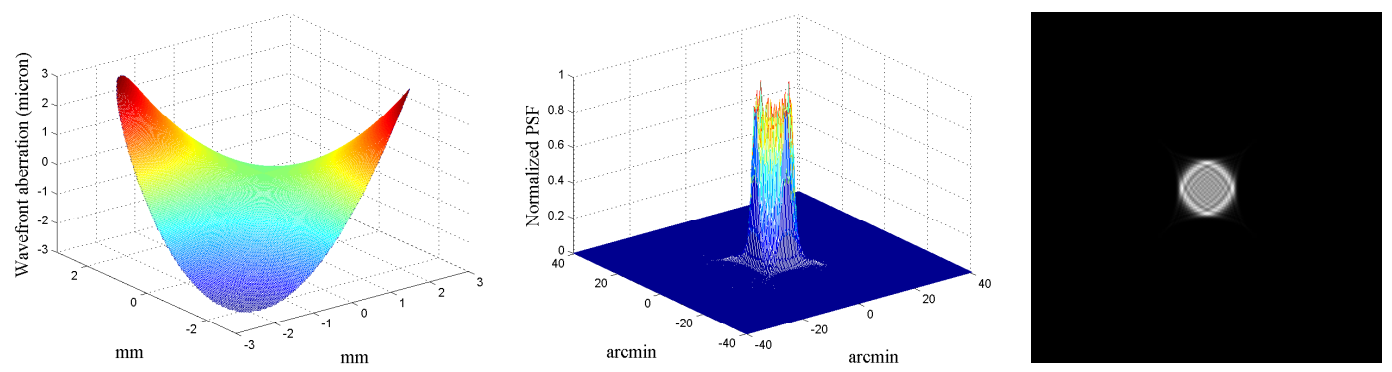

(b)
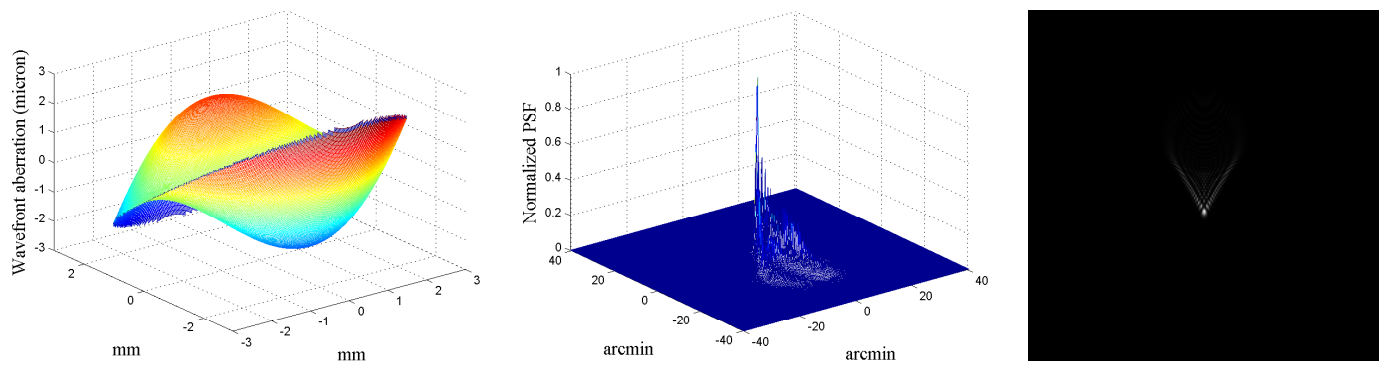

(c)
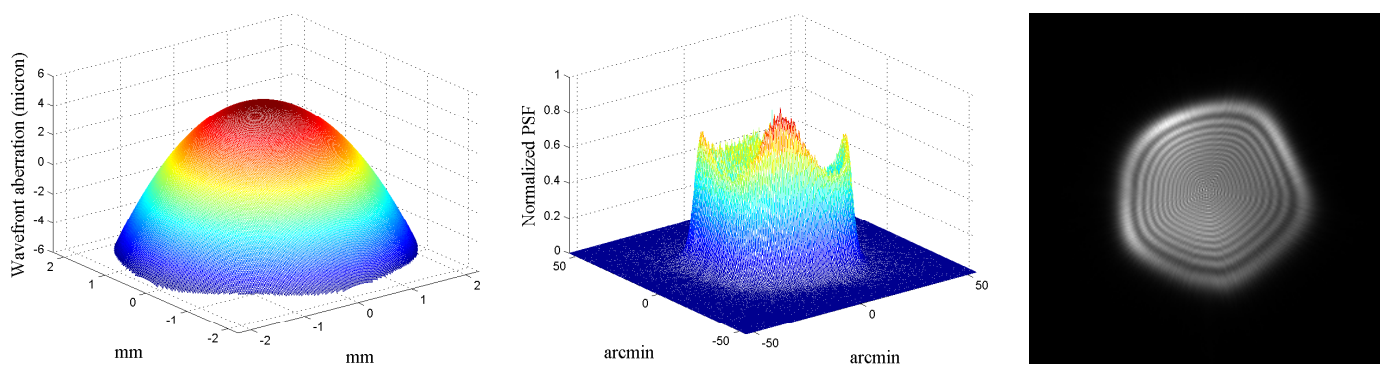

(d)

Figure 3.1: Examples of wavefront aberration function and normalized PSF corresponding to (a) $-1 \mu m$ of defocus aberration, (b) $-1 \mu m$ of astigmatism aberration, (c) $-1 \mu m$ of coma aberration and (d) a real eye with -4 Diopter of spherical error and $4.5 \mathrm{~mm}$ pupil diameter. 
becomes larger. More interestingly, the distribution extent of the PSFs exhibit considerable differences among different Zernike modes. With the same wavefront error $(-1 \mu m)$, defocus aberration generates the broadest PSF distribution, followed by the astigmatism, and the narrowest is the coma, which is a high order aberration. This implicates that low order aberrations (e.g., defocus and astigmatism) may have a larger impact on the visual performance than high order aberrations such as coma.

\subsubsection{Retinal Image Formation as Convolution}

The PSF is a very useful tool to describe the visual performance of the eye. Using the PSF for modeling the viewing process, the retinal image becomes accessible and the impact of aberrations on the visual performance can be evaluated in a straightforward way. If the object viewed is considered as a collection of point sources in two dimension, the formation of retinal image can be simplified as an integration of the superimposed distribution from the corresponding point sources. Therefore, mathematically, the retinal image formed can be described as the convolution of the spatial object intensity and the PSF of the eye, as shown in Figure 3.2.

An important feature of the wavefront aberration is that it contains all optical defects of the human eye, including the defects caused by the cornea and the lens.

Likewise, the PSF describes the image formation pattern of the eye as a whole, taking account of all the aberrations as well as the distortion caused by diffraction. This means we can take the optical system of the eye as a black box and predict the retinal image as its output. 


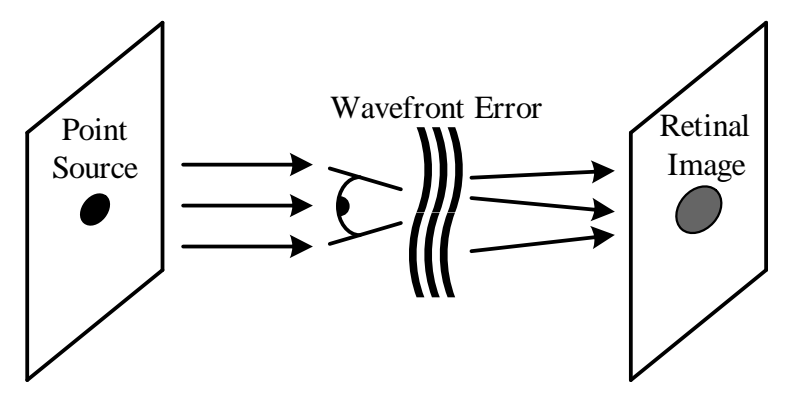

(a)

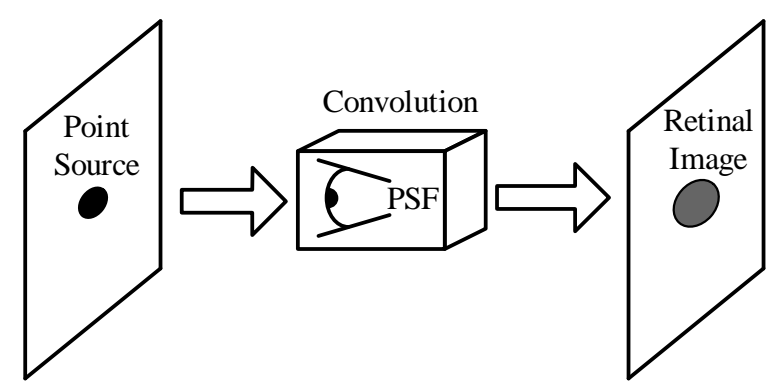

(b)

Figure 3.2: Retinal image formation as (a) wavefront propagation and (b) convolution process with the Point Spread Function of the eye.

\subsubsection{Modulation Transfer Function}

The counterpart of the PSF in the frequency domain is called Optical Transfer Function (OTF). The OTF and the PSF are a Fourier transform pair, which means they can be derived form each other by Fourier transform

$$
O T F=\mathcal{F}\{P S F\}
$$

The OTF is a complex-valued function of spatial frequency with phase information. The magnitude component of OTF is called Modulation Transfer Function (MTF), which describes the ability of an optical system to transfer object to image at various spatial frequencies. For the human eye, the MTF reveals how faithfully the image is formed by the eye's optical system, in a similar way to the frequency response of a linear system. Figure 3.3 shows the three-dimensional MTFs corresponding to the wavefront aberrations in Figure 3.1. It is found that the MTF becomes narrower as 


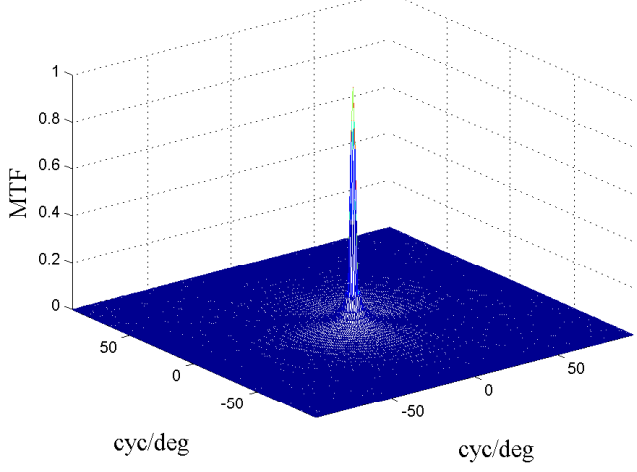

(a)

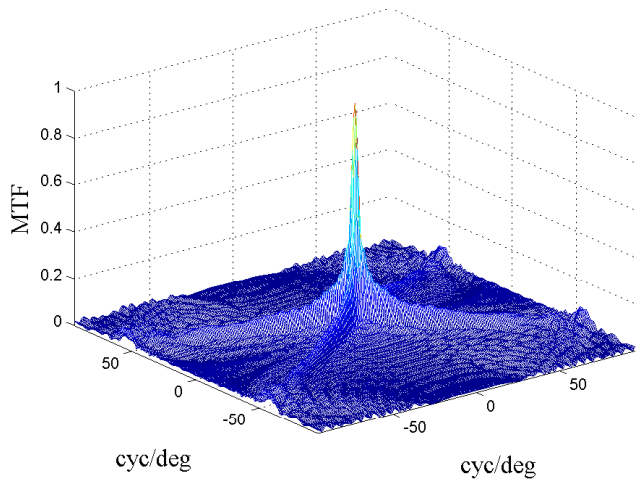

(c)

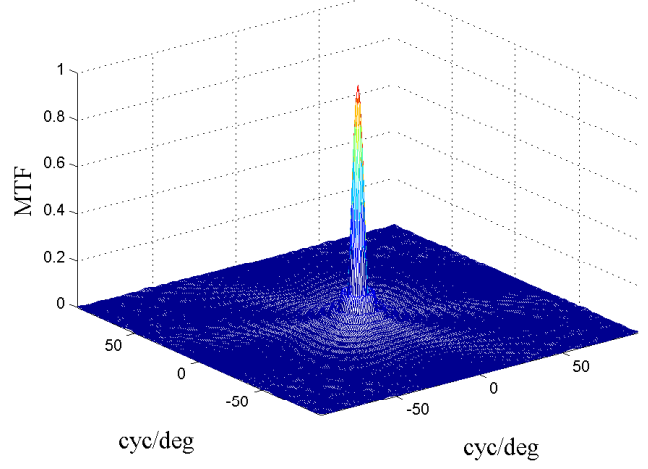

(b)

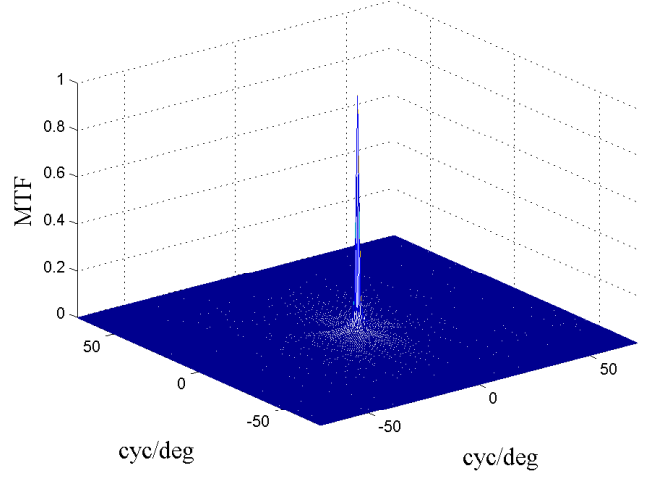

(d)

Figure 3.3: MTFs corresponding to (a) $-1 \mu m$ of defocus aberration, (b) $-1 \mu m$ of astigmatism aberration, (c) $-1 \mu m$ of coma aberration and (d) a real eye with -4 Diopter of spherical error and $4.5 \mathrm{~mm}$ pupil diameter.

the PSF becomes broader, which means that the eye's ability to recognize the high frequency spatial information decreases. Along with the PSF, the MTF will be used to characterize the ocular aberrations of computer users in the modeling of image precompensation.

\subsubsection{Limitation of Retinal Image}

In general, the image quality of an optical system is mainly degraded by its wavefront aberration and diffraction pattern. The impact of diffraction on the image quality always exists and can not be avoided. For most optical systems, the impact caused 


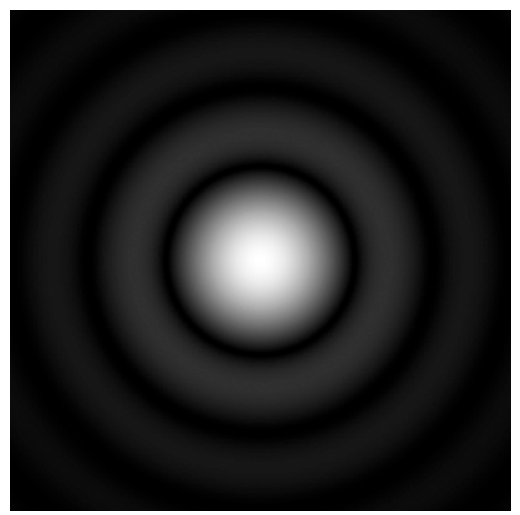

(a)

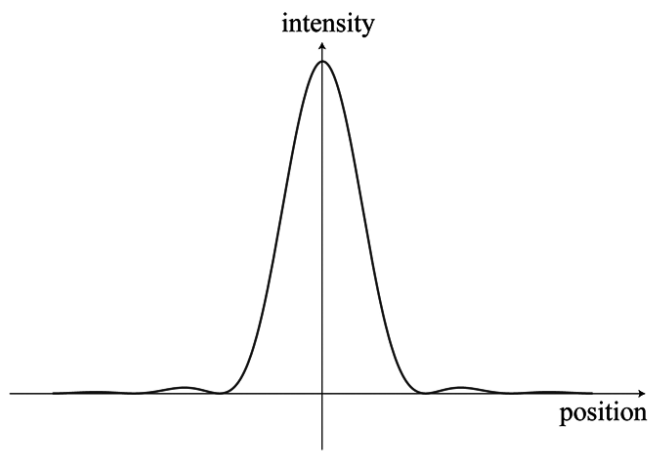

(b)

Figure 3.4: Airy disk pattern in (a) gray scale image and (b) cross section. The angular radius of the first diffraction ring is related to the diameter of the aperture.

by diffraction is relatively quite small and can be ignored, comparing to the impact of the aberration. However, for an ideal optical system without any aberration, diffraction becomes the only factor that limits the image quality, if the effect of scattering is not considered. Such ideal optical system is also called diffractionlimited system. The diffraction imposes restrictions on the highest frequency of the spatial information the optical system can handle, as well as the highest resolution of the optical system.

Due to the diffraction, the PSF of a point source produced by an aberration-free eye is not a point image, but a circular region with diffraction rings called Airy Disk, as shown in Figure 3.4. The size of Airy Disk is proportional to the wavelength and inversely proportional to the pupil diameter. The angular radius of the first ring that surrounds the bright central disk is given by

$$
r_{d i s k}=1.22 \frac{\lambda}{D}
$$

in cyc/radian, where $\lambda$ is the wavelength of the light and $D$ is the diameter of the pupil. The Airy Disk determines the minimum angular information that can be recognized by the eye. According to the Rayleigh criterion [21], two image points 


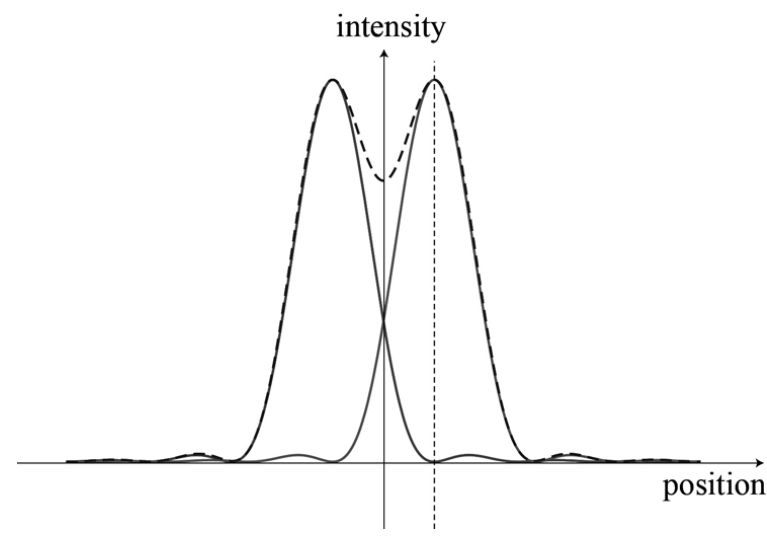

Figure 3.5: Rayleigh criterion that limits the resolution of imaging systems.

are regarded as "just resolved" when the peak of Airy pattern of one point falls right on the top of the valley of Airy pattern of the other point, as shown in Figure 3.5.

The limitation of diffraction can also be demonstrated with the MTF. For a diffraction-limited eye with no aberration, the value of the MTF at spatial frequency $v$ is given by

$$
\operatorname{MTF}(v)=\frac{2}{\pi}(\phi-\sin \phi \cos \phi),
$$

where

$$
\phi=\arccos \frac{\lambda v}{D} .
$$

From Equation (3.6), it is found that the magnitude of MTF gradually decreases as the spatial frequency increases, and finally reduces to zero at a certain point. The frequency at this point, called cutoff frequency, is given by

$$
f_{c}=\frac{D}{\lambda} .
$$

Thus, the highest frequency components of the image formed on the retina is $f_{c}$. Spatial components with higher frequency than $f_{c}$ will be distorted by the distribution of the diffraction. For a wavelength of $550 \mathrm{~nm}$, the highest spatial frequencies that can be viewed by a diffraction-limited eye with pupil diameter of $3 \mathrm{~mm}, 5 \mathrm{~mm}$ 


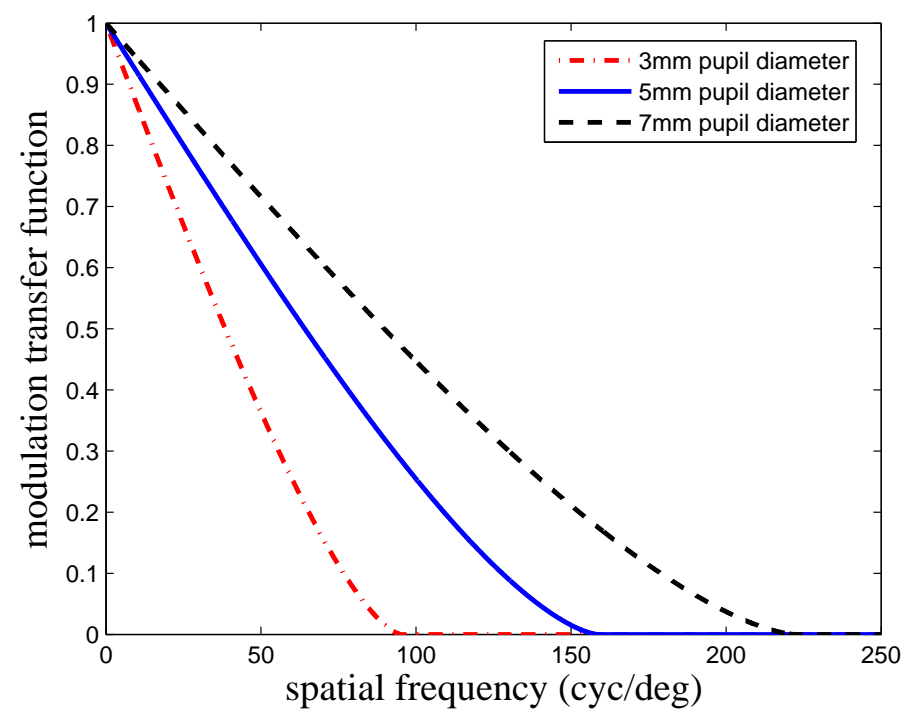

Figure 3.6: MTF curves of aberration-free eyes with pupil diameter of $3 \mathrm{~mm}, 5 \mathrm{~mm}$ and $7 \mathrm{~mm}$. The cutoff frequency of the MTF increases when the pupil size becomes larger.

and $7 \mathrm{~mm}$, are 95, 158, and $222 \mathrm{cyc} /$ degree, respectively. The MTF curves of the ideal eyes with those different pupil sizes are shown in Figure 3.6.

With the PSF and MTF, we have demonstrated that the diffraction limitation of the eye is related to the wavelength of the light and the pupil diameter. Interestingly, while the ocular aberration tends to increase with pupil size, the eye with a larger pupil is capable of recognizing more detailed spatial information.

\subsubsection{Viewing Distance and Aberration}

As we introduce in Chapter 2, the wavefront aberration is defined as the difference between the actual wavefront and the referenced wavefront. Usually, the wavefront aberration measured by the wavefront aberrometers is obtained with respect to a plane wave. For the human eye, it is assumed that the eye is viewing a target at an infinite distance during the measurement. The measured ocular aberration is 


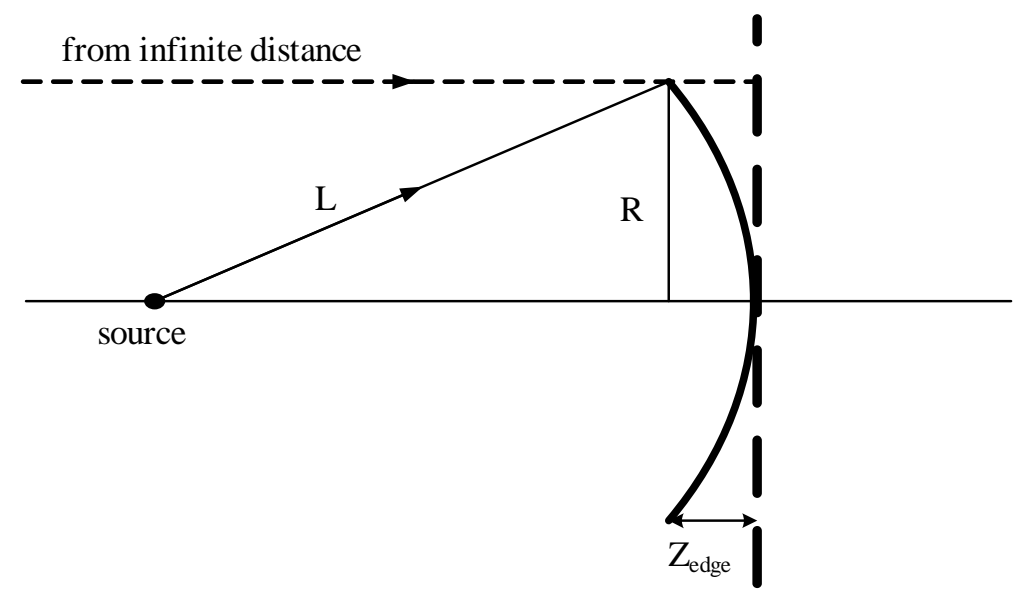

Figure 3.7: Geometry demonstrating the wavefront deviation between the plane wavefront originated from an infinite distance and spherical wavefront originated from a finite distance.

close to the actual aberration when the eye views remote targets at great distances. However, this approximation may be inadequate in the context of computer use, in which the viewing distance is typically less than one meter. Therefore, the wavefront deviation caused by the finite viewing distance should be taken into consideration in the actual image viewing model.

In contrast to viewing a target at infinite distance, the wavefront originated from a target at a finite distance does not propagate to the pupil in a plane wave, but in a spherical wave related to the finite viewing distance. The wavefront deviation due to the viewing distance can be demonstrated by the difference between the plane surface and the spherical surface, as shown in Figure 3.7. From Figure 3.7, the maximum deviation in the wavefront surface $Z_{\text {edge }}$, which is located at the edge of pupil, can be derived as

$$
Z_{\text {edge }}=L-\sqrt{L^{2}-R^{2}}
$$

based on the Pythagorean theorem, where $L$ is the viewing distance and $R$ is the radius of the pupil. 
In practice, instead of adding the wavefront deviation due to the viewing distance to the reconstructed wavefront aberration, this wavefront deviation can be integrated into the measured Zernike coefficients, which represent the wavefront errors in different Zernike modes. More specifically, this wavefront deviation can be added to the Zernike coefficient of the defocus mode to generate a new coefficient that also accounts for the viewing distance, since the shape of defocus aberration is also spherical. In fact, viewing distance is inherently related with the defocus aberration. This can be verified, intuitively, by the fact that spherical error in a myopic eye will disappear if the viewing target is close enough.

Due to the orthogonality of Zernike polynomials, the measured defocus aberration $W_{\text {def }}$ can be calculated as

$$
W_{\text {def }}=Z_{\text {def }} \sqrt{3}\left(2 \rho^{2}-1\right)
$$

where $Z_{\text {def }}$ is the defocus Zernike coefficient and $\sqrt{3}\left(2 \rho^{2}-1\right)$ is the corresponding Zernike polynomial. Because the wavefront deviation $Z_{\text {edge }}$ is effective only at the edge of the pupil, the average wavefront error due to the viewing distance $W_{\text {dist }}$ can be approximately represented as

$$
W_{\text {dist }}=Z_{\text {dist }} \sqrt{3}\left(2 \rho^{2}-1\right)
$$

where

$$
Z_{\text {dist }}=\frac{Z_{\text {edge }}}{2}
$$

is the equivalent Zernike coefficient if the impact of viewing distance is considered. Combining the measured defocus aberration $W_{\text {def }}$ and the wavefront error due to the viewing distance $W_{d i s t}$, the new defocus aberration $W_{d e f}^{\prime}$ is generated as

$$
W_{d e f}^{\prime}=Z_{d e f}^{\prime} \sqrt{3}\left(2 \rho^{2}-1\right)
$$


where $Z_{\text {def }}^{\prime}$ is the new defocus Zernike coefficient with viewing distance adjustment, represented as

$$
Z_{\text {def }}^{\prime}=Z_{\text {def }}+Z_{\text {dist }}
$$

This adjusted Zernike coefficient will be used to calculate the actual ocular aberration used to build the precompensation model. The ocular aberrations and PSFs of an aberrated eye (Spherical: -4.5 D, Cylindrical: -0.6 D, Axis: 147 ${ }^{\circ}$ ) before and after viewing distance adjustment are compared in Figure 3.8. From it, we can find that the originally measured ocular aberration is reduced and the corresponding PSF becomes sharper as we add the viewing distance adjustment into its defocus component. The adjusted ocular aberration gets closer to the original aberration when the viewing distance is increased, as shown in Figure 3.8(d).

\subsubsection{Computation of PSF}

When a person views an image on the computer screen, the corresponding retinal image can be predicted by the convolution of the PSF of the eye and the displayed image, as shown in Figure 3.2. Careful preprocessing and calibration of the PSF is required before the convolution, in order to match the resolution and field of view (FOV) of the displayed image on the screen.

In this study, the PSF of the computer user's eye is derived from the ocular aberration, which is available through the measurement by the aberrometer. The measured aberration is usually reported as a set of Zernike coefficients, representing the wavefront error of the corresponding Zernike modes. With the Zernike coefficients and corresponding pupil size, the numerical ocular aberration can be

reconstructed using Equation (3.1). Before the calculation of the PSF through it, 

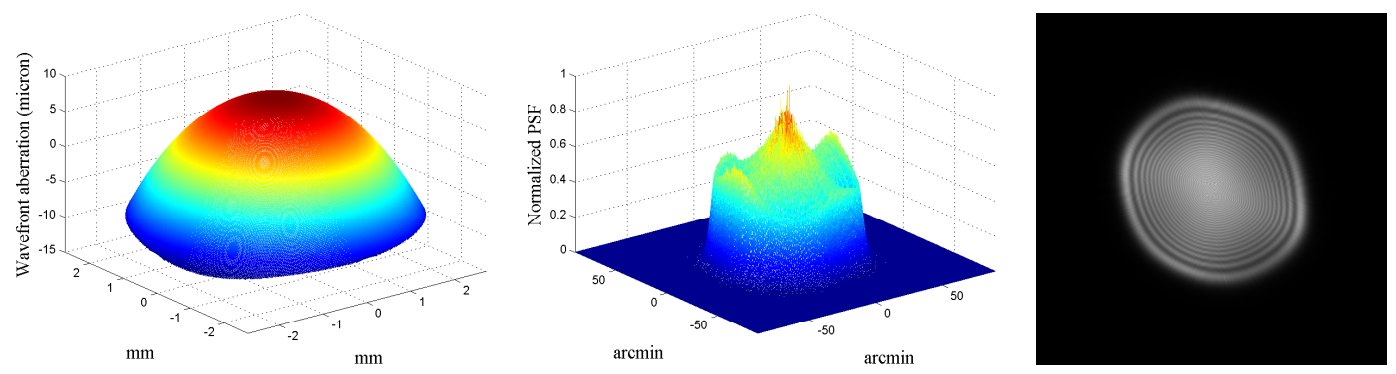

(a)
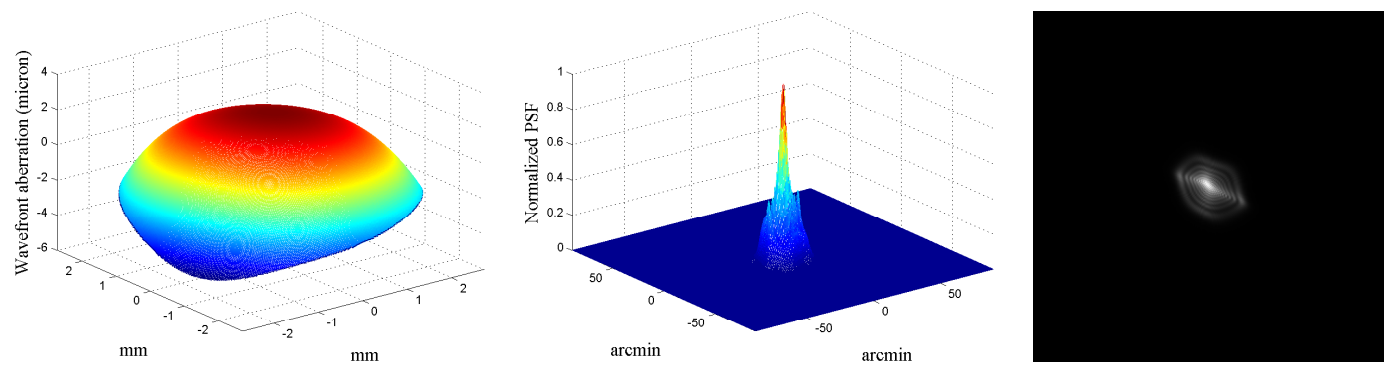

(b)
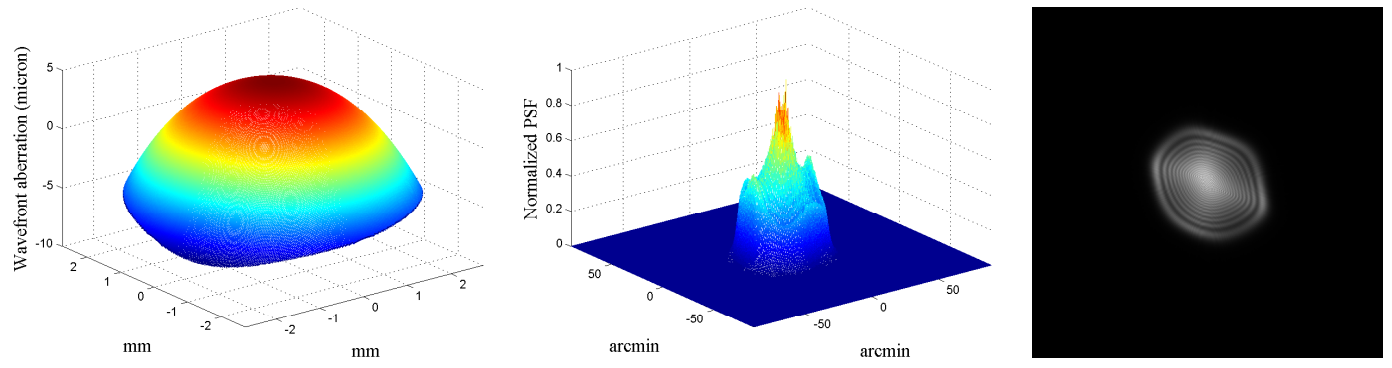

(c)
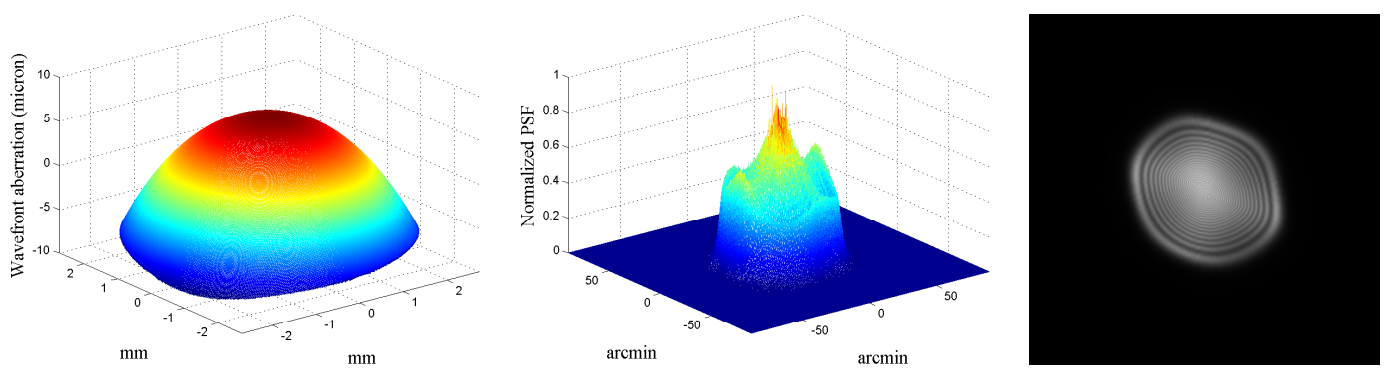

(d)

Figure 3.8: Comparison of ocular aberrations and normalized PSFs (a) without viewing distance adjustment, (b) with $0.3 \mathrm{~m}$ viewing distance adjustment, (c) with $0.5 \mathrm{~m}$ viewing distance adjustment and (d) with $1.0 \mathrm{~m}$ viewing distance adjustment. The original ocular aberration is measured from an eye with -4.5 D Spherical error and -0.6 D Cylindrical error with $147^{\circ}$ Axis. The pupil diameter of the eye is 5.4 $\mathrm{mm}$. 
the reconstructed aberration needs to be discretely sampled, with a resolution of $N_{p} \times N_{p}$

Usually, the calculated PSF, also with a resolution of $N_{p} \times N_{p}$, obtained from the ocular aberration, is not ready for convolution and needs to be calibrated according to the angular resolution of the eye and physical size of the displayed image. The criterion for calibration is to make sure that the FOV spanned by the PSF matrix is equivalent to the FOV when the displayed image is viewed.

The FOV of the displayed image is dependent on its physical size presented on the screen and the viewing distance of the observer. Suppose the resolution of displayed image is $M \times N$, the vertical FOV of the displayed image can be calculated as

$$
\operatorname{Fov}_{v}=2 \arctan \frac{d_{p} M}{2 L},
$$

in radians, where $d_{p}$ is the pixel pitch of the display device that defines the physical distance between two pixels and $L$ is the distance between the center of the displayed image and the observer's eye, as shown in Figure 3.9. The horizontal FOV of the displayed image $\mathrm{Fov}_{h}$ can be obtained in the same way as

$$
\text { Oov }_{h}=2 \arctan \frac{d_{p} N}{2 L} .
$$

The FOV of the PSF is related to the angular resolution of the eye, which is determined by the diffraction pattern of the optical system. More specifically, the angular resolution of the eye can be represented by the full width at half maximum (FWHM) of the airy diffraction pattern of the PSF. Thus, the angular resolution of the eye $f_{r}$ is approximately given by

$$
f_{r}=\frac{\lambda}{D}
$$

where $\lambda$ is the wavelength of the light and $D$ is the pupil diameter. This angular resolution is consistent with the cutoff frequency of the MTF. As the PSF matrix is 


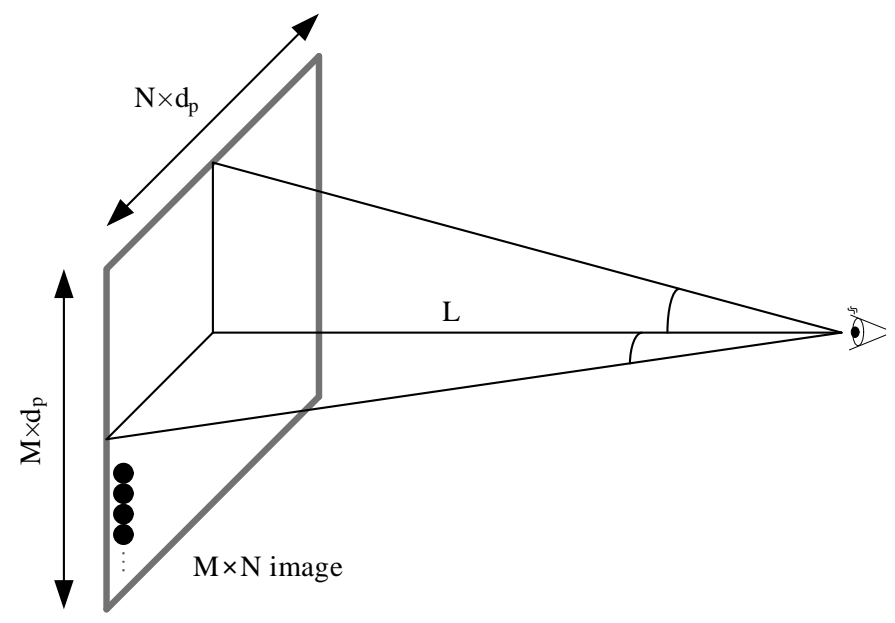

Figure 3.9: Geometry demonstrating the field of view of the eye when viewing the displayed image.

originally calculated through the Fourier transform of the eye's wavefront aberration, the size of the original PSF matrix is same as the sampled aberration. Therefore, the FOV of the original PSF matrix is determined by the sampling resolution of the eye's wavefront aberration, as each pixel's FOV in the PSF matrix equals to the angular resolution of the eye. In order to match the FOV of the PSF to the FOV of the displayed image, the PSF matrix needs to be calibrated according to the following

$$
F_{o v}=\eta_{v} f_{r} N_{p}
$$

and

$$
\text { Fov }_{h}=\eta_{h} f_{r} N_{p}
$$

in the vertical and horizontal dimensions, respectively, where $\eta_{v}$ and $\eta_{h}$ are the calibration factors of the PSF and $N_{p} \times N_{p}$ is the size of the PSF matrix before calibration. Thus, the vertical dimensional calibration factor $\eta_{v}$ is given by

$$
\eta_{v}=\frac{F o v_{v} D}{\lambda N_{p}}
$$




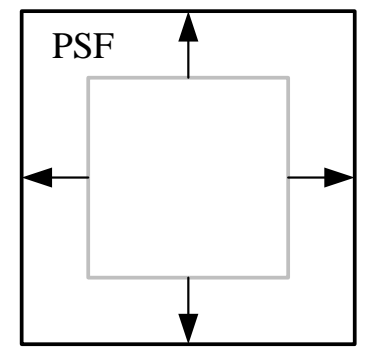

zero pad

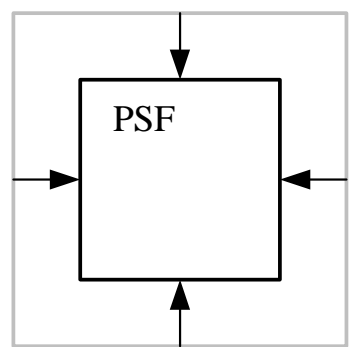

frame cut

Figure 3.10: Calibration of the PSF before the convolution. The field of view of the PSF matrix is enlarged by the zero padding operation and reduced by the frame cut operation.

and the horizontal dimensional calibration factor $\eta_{h}$ is given by

$$
\eta_{h}=\frac{\operatorname{Fov}_{h} D}{\lambda N_{p}}
$$

Suppose the calibration factor $\eta_{v}$ is larger than one, which means the vertical FOV of the PSF matrix is narrower than the displayed image, we need to pad extra

$$
n_{\text {pad }}=\frac{\left(\eta_{v}-1\right) N_{p}}{2}
$$

rows of zero in the top as well as the bottom of the PSF matrix, increasing the number of pixels in the vertical dimension to $\eta_{v} N p$. If the calibration factor $\eta_{v}$ is smaller than one, which means the vertical FOV of the PSF matrix is wider than the displayed image, it will be necessary to cut

$$
n_{c u t}=\frac{\left(1-\eta_{v}\right) N_{p}}{2}
$$

rows of pixels at the top as well as the bottom of the PSF matrix, reducing the number of pixels in the vertical dimension to $\eta_{v} N p$. The calibration of the PSF matrix in the horizontal dimension can be manipulated in the same way. The zero padding and frame cut operations applied to the PSF matrix are demonstrated in Figure 3.10. In practice, the calibration factors are usually larger than one. If the 
size of the wavefront aberration equals to the size of the displayed image, calibration factors smaller than one are required only when the display resolution is beyond the resolving ability of the eye.

The calibrated PSF matrix is then resized to the same size as the displayed image. Now, the FOV of each pixel in the PSF matrix becomes equivalent to the FOV of the pixel in the displayed image. Therefore, for an aberration-free eye, the outcome of convolution will be identical to the displayed image without any distortion, as its PSF matrix is a two-dimensional Delta function. For simplicity, the PSF matrix is usually normalized in terms of the intensity before convolution. Figure 3.11 shows an example in which the FOV of the PSF is doubled after calibration.

\subsubsection{Simulation of What Computer Users View}

After the calibration of FOV, the PSF is prepared to be convolved with the image displayed on the screen to simulate what the computer user views. The convolution operation of two images can be simplified greatly if these two images have the same resolution, as in this case the convolution outcome can be obtained through the simple multiplication of the Fourier transforms of these two matrices. Therefore, the calibrated PSF matrix is always resized to the same size as the displayed image before it is convolved with it. It should be noted that both the defocus aberration adjustment and the FOV calibration factor are related with the viewing distance between the eye and the displayed image. For the same eye, the corresponding PSFs used for the convolution varies with the changing of viewing distance, thereby resulting in different retinal images and visual perceptions. This is consistent with our daily experiences and easy to understand. Figure 3.12 shows the PSFs of an aberrated eye (Spherical: -5.8 D, Cylindrical: -1.8 D, Axis: $2^{\circ}$ ) at three different 


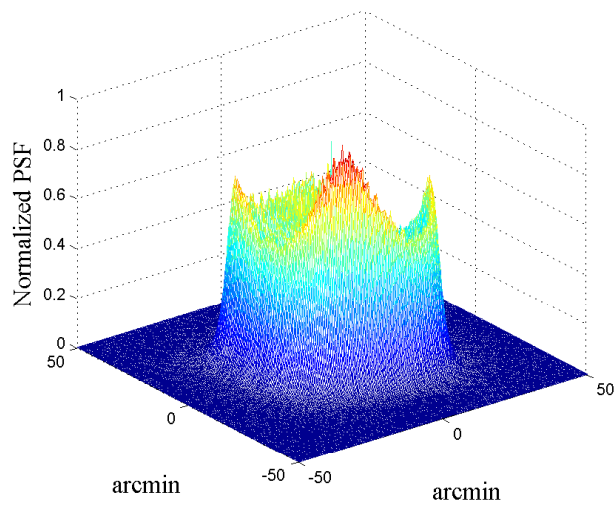

(a)

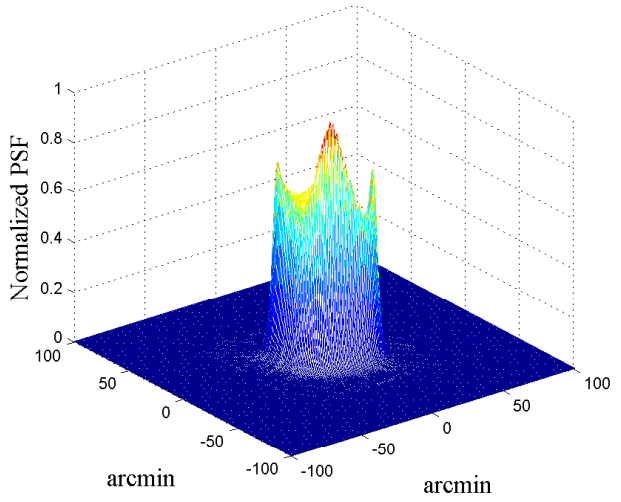

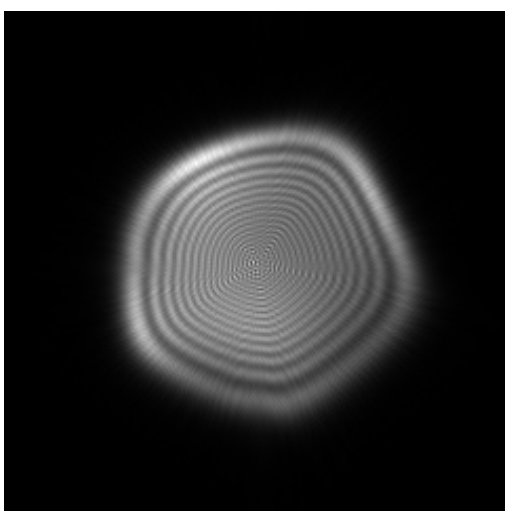

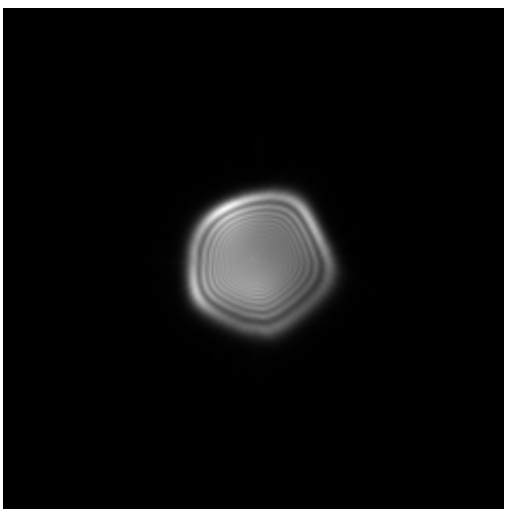

(b)

Figure 3.11: Example of PSF calibration in which the FOV of the PSF is increased from (a) 100 arcminute to (b) 200 arcminute, in both horizontal and vertical dimensions.

viewing distances of $0.3 \mathrm{~m}, 0.5 \mathrm{~m}$ and $0.8 \mathrm{~m}$. These PSFs have been adjusted and calibrated according to the viewing distance and the physical size of the displayed image, thereby ready to be convolved with the image to simulate the viewing process. Figure 3.13 shows the simulated retinal images obtained using the PSFs illustrated in Figure 3.12, which have been adjusted to correspond to different viewing distances. The pupil diameter of the eye here is $4 \mathrm{~mm}$. Obviously, the visual images become more blurred as the viewing distance becomes larger. When the viewing distance is $0.8 \mathrm{~mm}$, the image blurring is severe enough that even the most basic shape information of the images can not be recognized. 

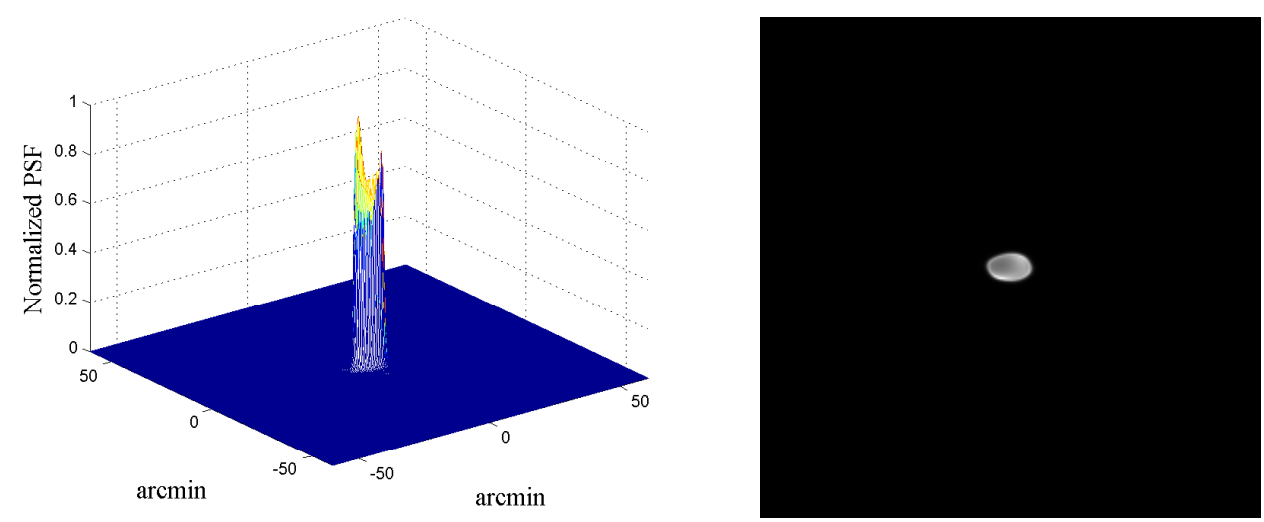

(a)
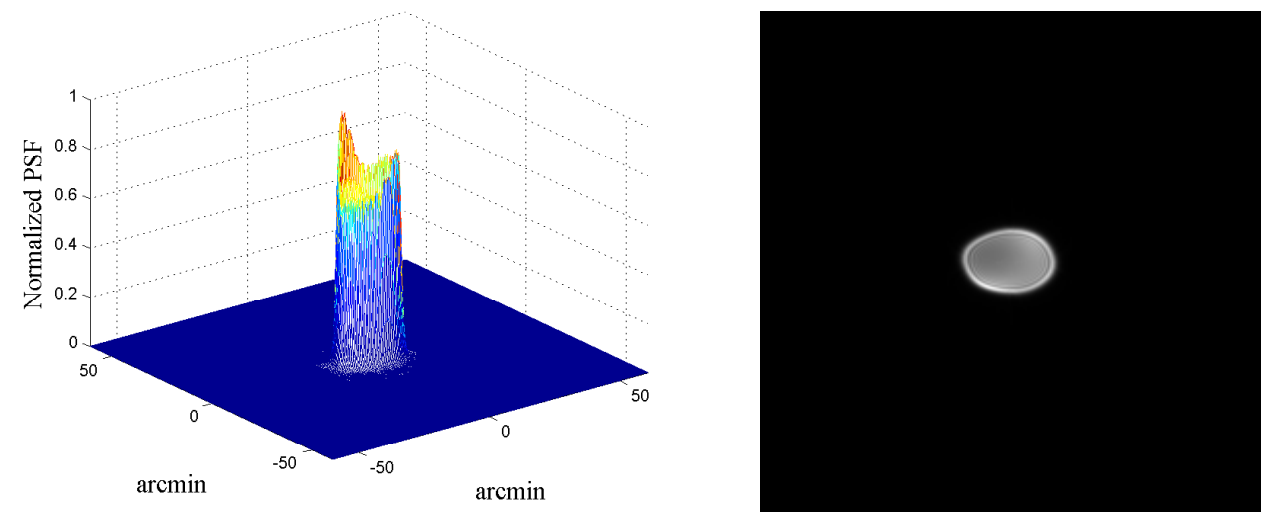

(b)
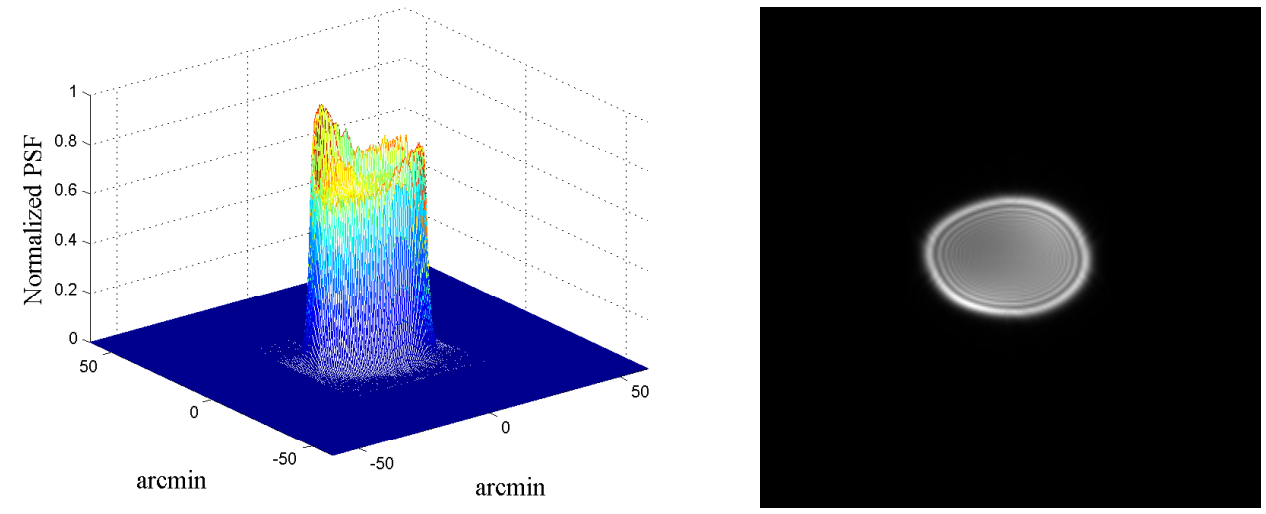

(c)

Figure 3.12: PSFs of an aberrated eye with -5.8 D Spherical error and -1.8 D Cylindrical error with $2^{\circ}$ Axis at viewing distance of (a) $0.3 \mathrm{~m}$, (b) $0.5 \mathrm{~m}$ and (c) 0.8 $\mathrm{m}$, respectively. The PSFs here have been calibrated according to the the viewing distance and the physical size of the displayed image. 

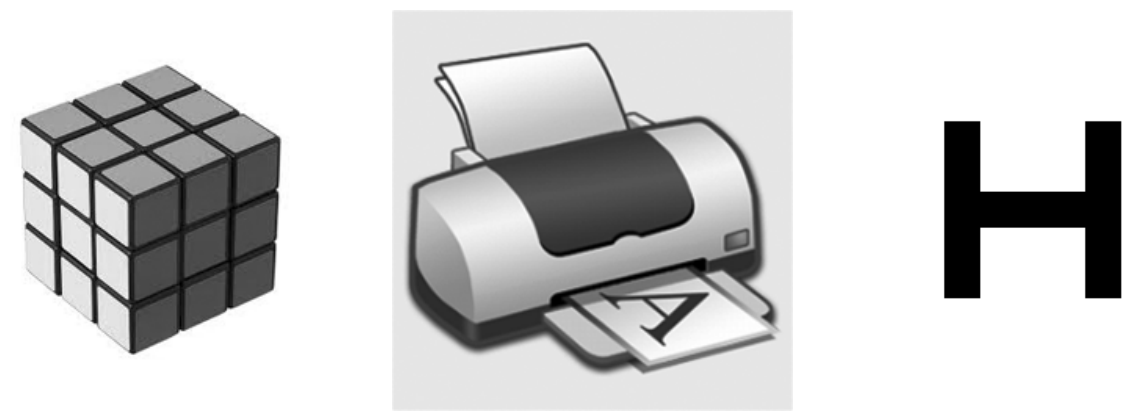

(a)
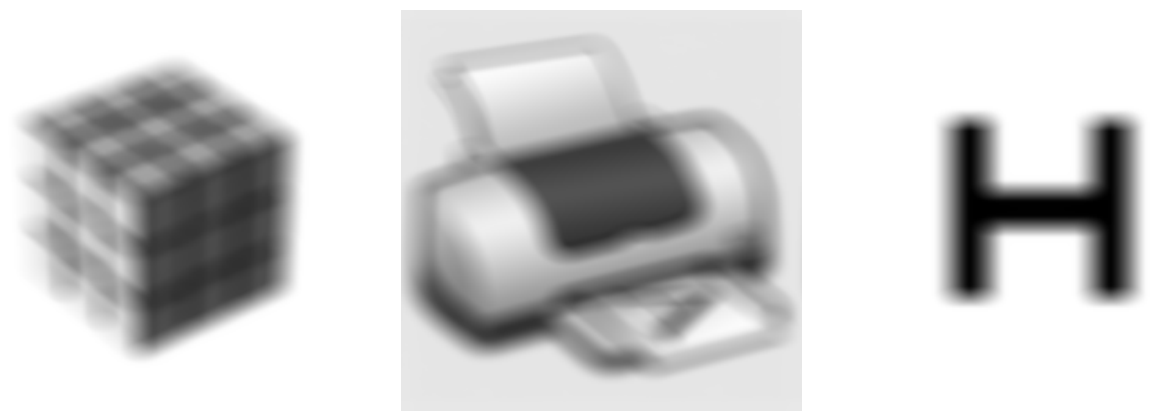

(b)
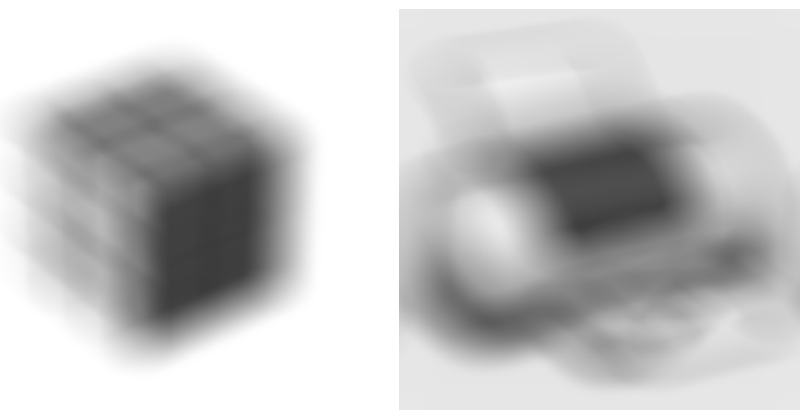

(c)
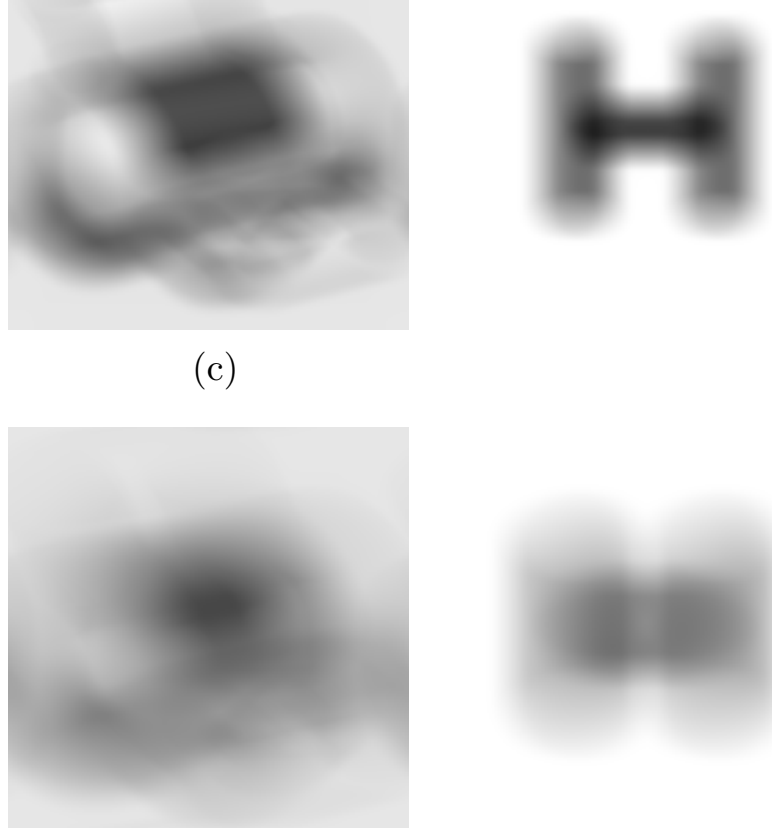

(d)

Figure 3.13: Simulation of retinal images viewed by an aberrated eye with $-5.8 \mathrm{D}$ Spherical error and -1.8 D Cylindrical error with $2^{\circ}$ Axis: (a) Original displayed images, (b) Simulated images viewed at distance of $0.3 \mathrm{~m}$, (c) Simulated images viewed at distance of $0.5 \mathrm{~m}$ and (d) Simulated images viewed at distance of $0.8 \mathrm{~m}$. 


\subsection{Image Precompensation of Aberration}

All the graphical information (e.g., pictures, menu, icons) displayed by modern computers can be represented in the form of digital images. For computer users with normal vision, the perception of the presented images is accomplished with satisfactory clearness and sharpness, as the images are designed and rendered with careful consideration. However, these well-designed images may not be fit for those people without normal vision. Due to the blurring in the perception of images, many computer users with severe ocular aberrations may encounter difficulties in identifying images, which hinders their further access to computer resources.

When a person views an image presented on the screen, the image blurring pattern perceived is determined by the observer's ocular aberration. More importantly, the blurred retinal image produced by the aberration can be predicted from the knowledge of the PSF, according to the linear modeling of the human visual system. Since the PSF and the MTF can be used to describe the imaging process of the eye in the spatial domain and frequency domain respectively, it is quite possible that the image blurring can be counterbalanced if an inverse compensation is inserted into the imaging process, just like the way we do to restore images affected by various types of degradation. Traditional vision correction methods like glasses and contact lenses add this compensation by adjusting the propagation of the wavefront before the light enters into the human eye. On the other hand, the computer, as one side of the interaction and the source of the imaging process, is capable of preprocessing the images and add personalized compensation based on the ocular aberrations of the observer. Thus, in this study, the original images for display are modified in particular ways with specific compensation, to overcome the image blurring caused by the ocular aberration of the computer user. 


\subsubsection{Comparison with Image Restoration}

The image precompensation problem in this study has similarities with non-blind image restoration. In general, the image restoration process assumes that the degradation model is known or can be estimated in reliable ways. Similarly, for image precompensation, the visual degradation model (PSF of the eye) of the specific computer user is required as a priori knowledge before the personalized compensation can be produced. On the other hand, image precompensation is substantially different from image restoration. Image restoration methods post-process the degraded image based on the known degradation information in order to restore the original image from its degraded version, whereas the image precompensation method modifies the original images in a particular fashion, in order to neutralize the visual blurring caused by the aberration of the viewer's eye.

Image restoration is a relatively mature research field with a long history. So far, numerous image restoration techniques have been developed to recover the undistorted images from a degraded version with different assumptions on the prior knowledge. The prior knowledge includes knowledge of the degradation, statistical information of the noise and the original image, etc. Usually, the process of image restoration will be greatly facilitated and simplified if the complete degradation model is known. Compared with image restoration, image precompensation is sub-

ject to some new problems and difficulties due to its specificity. One big challenge is that the image perceived by the user is not accessible. Thus, classical image restoration methods that involve iterative optimization $[37,73,75]$ are not applicable here due to the lack of reliable ways to update the estimation for convergence. Moreover, unlike image restoration, image precompensation of aberration requires real-time response. Thus, precompensation algorithms that require too many computations are also inadequate. In this study, we use the Inverse Blurring Filter (IBF) to produce 
the personalized compensation, since a relatively accurate ocular aberration can be measured through the wavefront aberrometer.

\subsubsection{Inverse Blurring Filtering}

Suppose an image with intensity $o(x, y)$ is to be viewed by a computer user. Because of the aberration existing in the user's eye, the retinal image generated is degraded with distortion, resulting in an intensity distribution on the retina. The degradation of the image can be described as a convolution operation:

$$
i(x, y)=o(x, y) * P S F(x, y)
$$

where $*$ is the convolution operator, $i(x, y)$ is the discrete retinal image with degradation and $P S F(x, y)$ is the PSF matrix of the eye after calibration. The OTF of the eye is derived by

$$
O T F(u, v)=\mathcal{F}\{P S F(x, y)\}
$$

as the Fourier transform of the PSF, where $u$ and $v$ are the horizontal and vertical spatial frequencies in discrete form. Based on its definition, the MTF is calculated as the modulus of the complex-valued OTF:

$$
\operatorname{MTF}(u, v)=|O T F(u, v)| .
$$

Applying Fourier transform to both sides of Equation (3.24), the imaging process with degradation can also be described in the frequency domain as

$$
I(u, v)=O(u, v) O T F(u, v) .
$$

Now, the problem is how to produce an image with appropriate compensation to replace the original image $o(x, y)$, to be presented to the viewer. One intuitive idea

is using inverse filtering, in which the $O(u, v)$ is divided by $O T F(u, v)$ in advance to 
counteract the blurring effects. Based on this idea, the compensated image $c(x, y)$ can be described by

$$
C(u, v)=\frac{O(u, v)}{O T F(u, v)}
$$

in the frequency domain. However, this is not practical since the measurement error of the ocular aberrations and noise during the imaging process would be strongly amplified by this form of inverse filtering, especially when $\operatorname{OTF}(u, v)$ is close to zero. In the worst case, the valid information of the compensated image will be buried by the amplified high frequency components, as the value of $O T F(u, v)$ tends to be very small in high frequencies. In such scenario, classic methods like Wiener filter are often used to suppress the pollution of high frequency components. Here, since the compensation is performed on the image source and not on the degraded image, an inverse filtering process called Inverse Blurring filter (IBF) is used to produce the desired compensated image. The compensation by IBF is described by

$$
c(x, y)=\mathcal{F}^{-1}\left\{\frac{O(u, v)}{O T F(u, v)} \frac{M T F(u, v)^{2}}{M T F(u, v)^{2}+K}\right\}
$$

where $\mathcal{F}^{-1}$ represents inverse Fourier transform and $K$ is a factor used to suppress the amplification of high frequency noise. Since no statistical information of the noise is available, here the factor $K$ is a small empirical constant. The compensated image $c(x, y)$ is generated to replace the original image $o(x, y)$, and be presented to the user on the screen. When it is viewed, the retinal image of the user can be predicted as

$$
p(x, y)=c(x, y) * P S F(x, y) .
$$

Figure 3.14 demonstrates the whole imaging process of generation of the retinal image, in which the image blurring caused by the aberration is counterbalanced by personalized precompensation. 


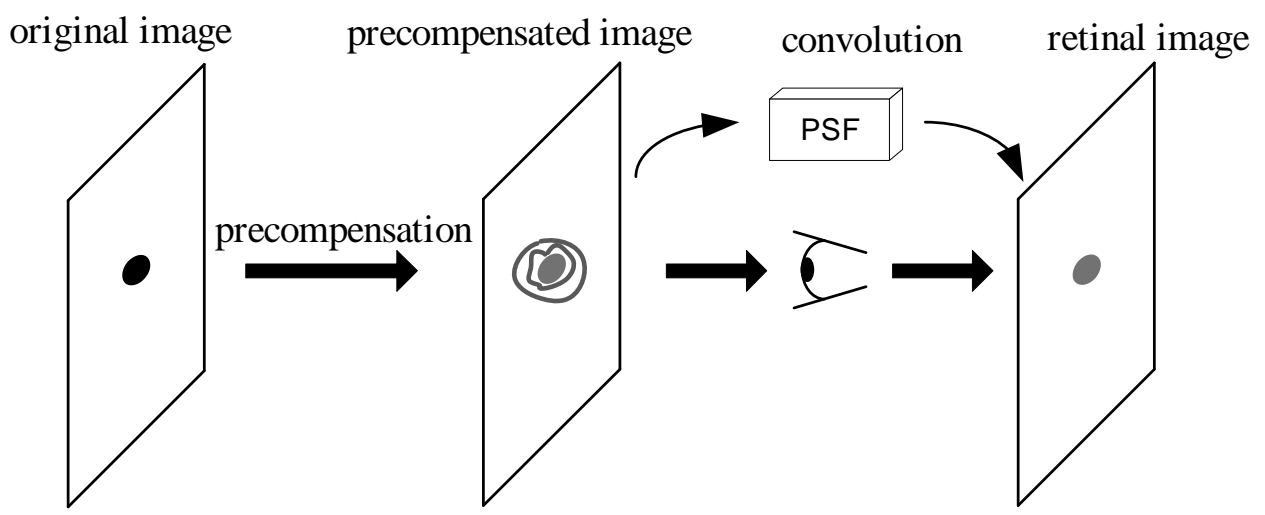

Figure 3.14: Schematic of the imaging process with precompensation. The original image is compensated to counteract the image blurring caused by the aberration.

\subsubsection{Contrast Loss of Precompensation}

The PSF of the human eye behaves like a low pass filter, blurring the high frequency details of the viewed images. In comparison, the precompensation process behaves like a high pass filter, even though it does not attenuate the low frequency components directly. While precompensation reduces the degradation caused by the eye's PSF, the compensated image usually has a wider intensity range than the original and may include negative components. One the other hand, display devices (e.g., LCD) only have limited intensity scales. Thus, the intensity values of the compensated image need to be shifted and scaled before they can be displayed. Suppose the intensity range of the compensated image $c(x, y)$ is $\left[c_{\min }, c_{\max }\right]$ and the range of the display device is $\left[d_{\min }, d_{\max }\right]$. In this study, the compensated image $c(x, y)$ is proportionally scaled and linearly shifted to fit the range of display device. After it, the new image for display is given by

$$
d(x, y)=\frac{\left(d_{\max }-d_{\min }\right)\left[c(x, y)-c_{\min }\right]}{c_{\max }-c_{\min }}+d_{\min }
$$

Define $\alpha$ as

$$
\alpha=\frac{d_{\max }-d_{\min }}{c_{\max }-c_{\min }}
$$


and define $\beta$ as

$$
\beta=d_{\min }-\frac{c_{\min }\left(d_{\max }-d_{\min }\right)}{c_{\max }-c_{\min }} .
$$

Then, the new retinal image $p^{\prime}(x, y)$ is predicted as

$$
p^{\prime}(x, y)=\alpha c(x, y) * P S F(x, y)+\gamma .
$$

where $\gamma$ is a constant defined as

$$
\gamma=\beta P S F(x, y) .
$$

Compared with $p(x, y)$ in Equation (3.30), it is found that the actual contrast of the retinal image has been compressed with a ratio determined by $\alpha$. In practice, the range $\left[c_{\min }, c_{\max }\right]$ is always much wider than $\left[d_{\min }, d_{\max }\right]$. Thus, it is obvious that $\alpha$ is much smaller than 1 . The smaller $\alpha$ is, the more contrast is lost.

\subsubsection{Software Simulation}

The simulation of the image precompensation process is demonstrated in Figure 3.15. The simulation results are based on the same eye used in Figure 3.13, with -5.8 D Spherical error and -1.8 D Cylindrical error with $2^{\circ}$ Axis. In this simulation, the pupil diameter is $4 \mathrm{~mm}$ and the viewing distance is set as $0.5 \mathrm{~m}$. Three images used for simulation are the logos of "Github", "Twitter" and "Android", respectively.

From the results shown in Figure 3.15, it is observed that the shapes and edges of the retinal images are much sharper after applying the precompensation. This confirms the potential of our method to relieve the visual blurring caused by ocular aberration. However, the process of image precompensation also introduces some side effects. Firstly, ringing artifacts are found in the retinal images after precompensation. This phenomenon is especially evident around the area near the edges

of the images. The ringing artifacts are caused by the regularization error in the 

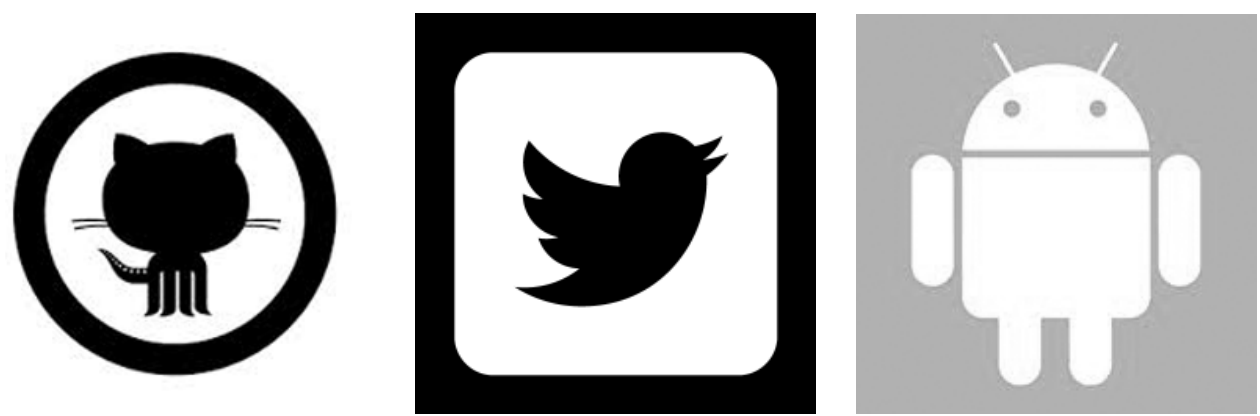

(a)
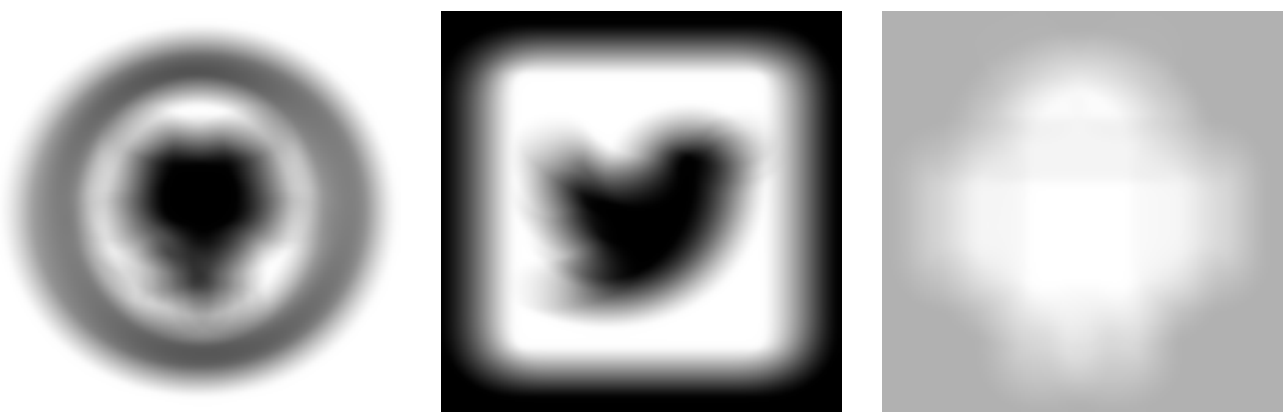

(b)
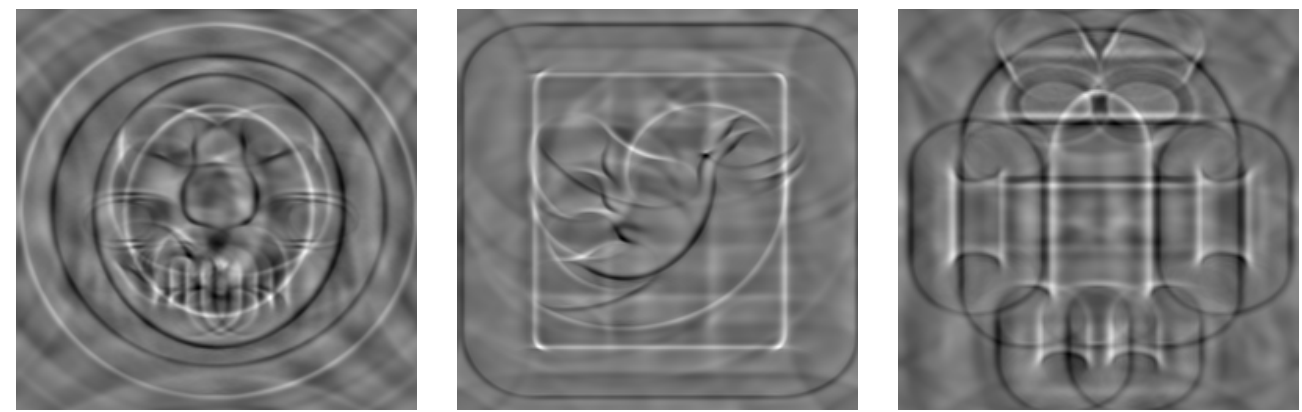

(c)
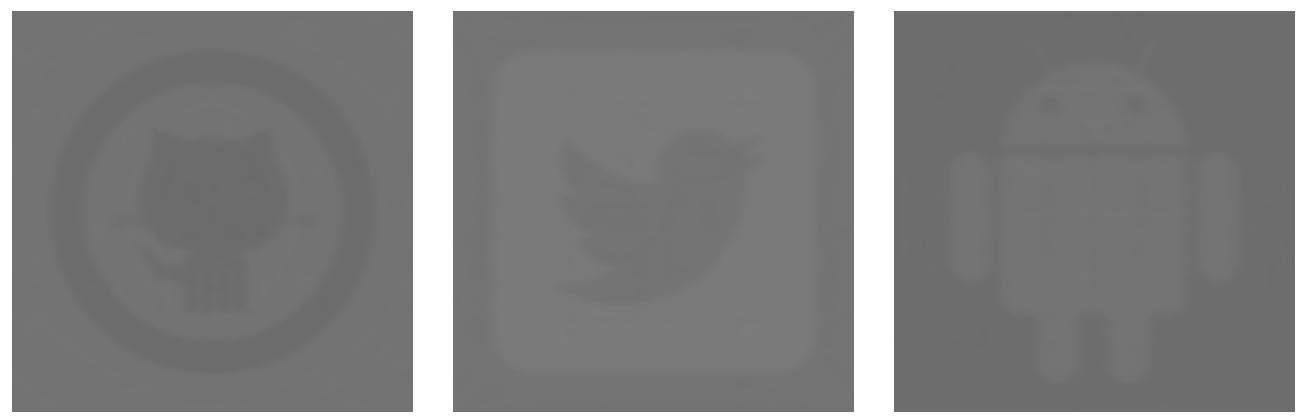

(d)

Figure 3.15: Simulation results of image precompensation based on an aberrated eye with -5.8 D Spherical error and -1.8 D Cylindrical error with $2^{\circ}$ Axis: (a) original displayed images, (b) retinal images viewed without precompensation, (c) precompensated images and $(\mathrm{d})$ retinal images viewed with precompensation. 
process of IBF, as a tradeoff with the suppression of the noise amplification. More importantly, it is noticed that the contrast of retinal images is significantly reduced after the compensation is applied. The contrast loss problem is illustrated more directly by the histograms of the images, as shown in Figure 3.16. While the pixels of the original images have widely separated gray levels, the gray levels of the simulated retinal images concentrate on a narrow band in the middle. It should be noted that the contrast of the retinal image is not directly dependent on the contrast of the original image. The original "Android" image, with less contrast than the other two, produces a retinal image with about the same contrast level as the other two after precompensation.

\subsubsection{Contrast Improvement by Histogram Clipping}

The contrast loss problem reduces the improvement of visual performance achieved through the precompensation method. In some extreme cases, the intensities of the whole compensated image will be packed together in a narrow band and the edge information of the image can hardly be recognized. Thus, particular enhancement methods need to be developed to increase the contrast of the retinal image.

Developing methods aiming to recover the original contrast is quite difficult since the contrast loss is fundamentally caused by the limited intensity range of the display devices. More specifically, the intensity range of the image after precompensation may have been extended to ranges that are beyond the display capability of regular display devices. Therefore, one more feasible strategy is to develop a contrast enhancement method to reduce the contrast loss and relieve the negative effects caused by the low contrast int the retinal image. 

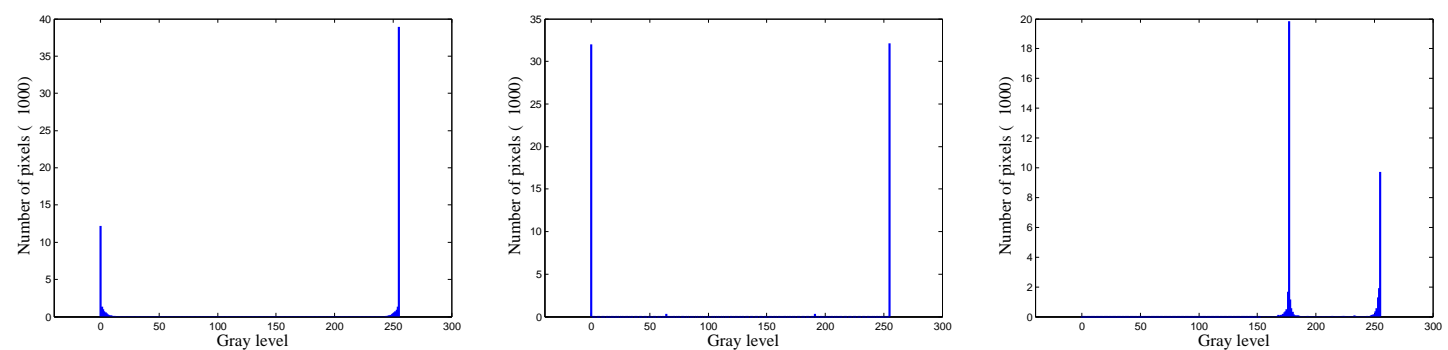

(a)
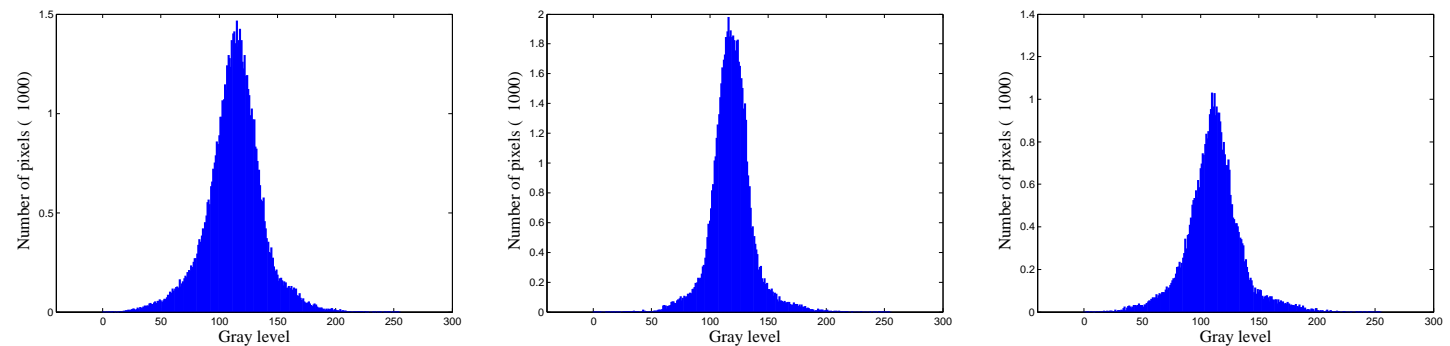

(b)
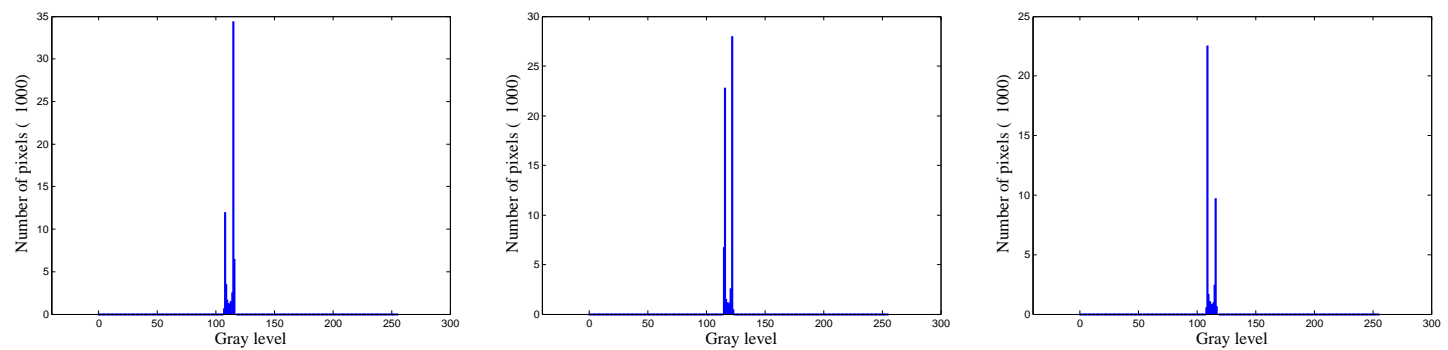

(c)

Figure 3.16: Histograms of the (a) original images, (b) precompensated images and (c) simulated retinal images.

In general, histogram equalization is an effective way to increase the contrast of images, especially the images with narrow histogram. However, basic histogram equalization is not appropriate to be used in our image precompensation method. Histogram equalization increases the image contrast by distributing the packed intensities to a wider range, based on the Cumulative Distribution Function (CDF) of the intensities of the image [20]. While the intensities of the image are spread out, the histogram shape of the compensated images may not be maintained, which, in this case, will distort the effect of the compensation. Other histogram based meth- 
ods like adaptive histogram equalization and contrast limited adaptive histogram equalization (CLAHE) [67], which are designed to improve local contrast, have similar problems.

In order to keep the shape of the histogram while spreading the intensities, we developed a histogram side-clipping method [28] to improve the contrast of the compensated image. From Figure 3.16, it is found that most intensity values of the compensated image concentrate around the background level, and comparatively few pixels have intensities located far above or far below the most frequent intensity values. This means that most of the compensation pattern is contributed by the intensities around the central band. Those intensities at both ends of the histogram have little impact on the overall effect of the compensation and can be ignored. Therefore, the histogram space occupied by the two sides can be used to extend the major part of the histogram, resulting in the increase of the contrast. The histogram side-clipping method in this study is described as:

$$
c^{\prime}(x, y)= \begin{cases}d_{\min } & \text { if } c(x, y)<c_{L} \\ \frac{\left[c(x, y)-c_{L}\right]\left[d_{\max }-d_{\min }\right]}{c_{H}-c_{L}}+d_{\min } & \text { if } c_{L} \leq c(x, y) \leq c_{H} \\ d_{\max } & \text { if } c(x, y)>c_{H}\end{cases}
$$

where $c^{\prime}(x, y)$ represents the enhanced compensated image, $c_{L}$ denotes the low-side cutoff intensity and $c_{H}$ denotes the high-side cutoff intensity. The low-side cutoff intensity and the high-side cutoff intensity are two thresholds that are used to clip the intensities in the histogram. Specifically, the intensities in the left side that are smaller than the low-side cutoff intensity will be assigned the minimum displayable level and the intensities in the right side that are larger than the high-side cutoff intensity will be assigned the maximum displayable level. In practice, these two thresholds can be automatically selected by analyzing the statistical information of the intensities of the original compensated image. 

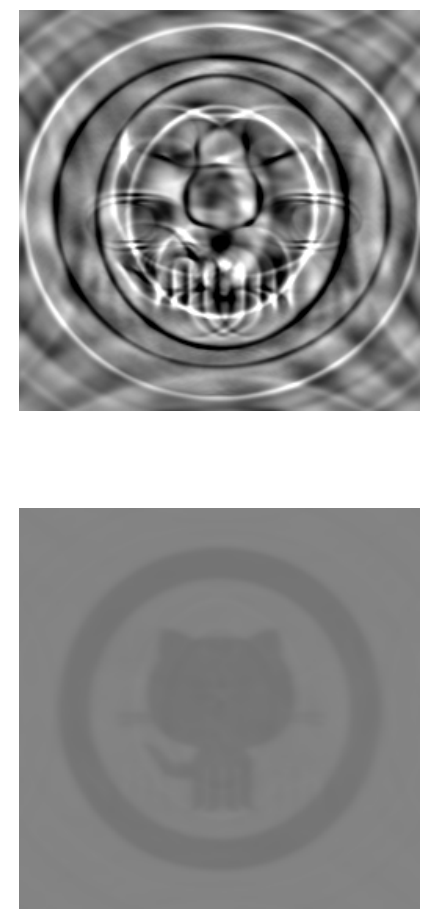

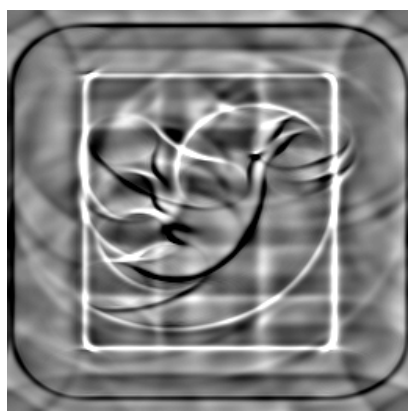

(a)

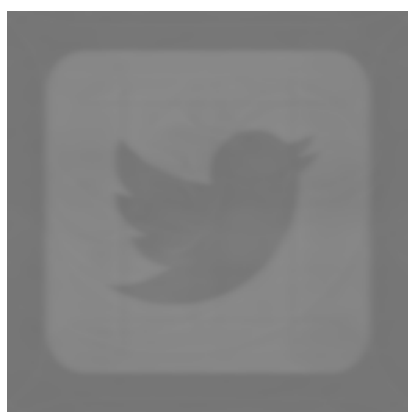

(b)
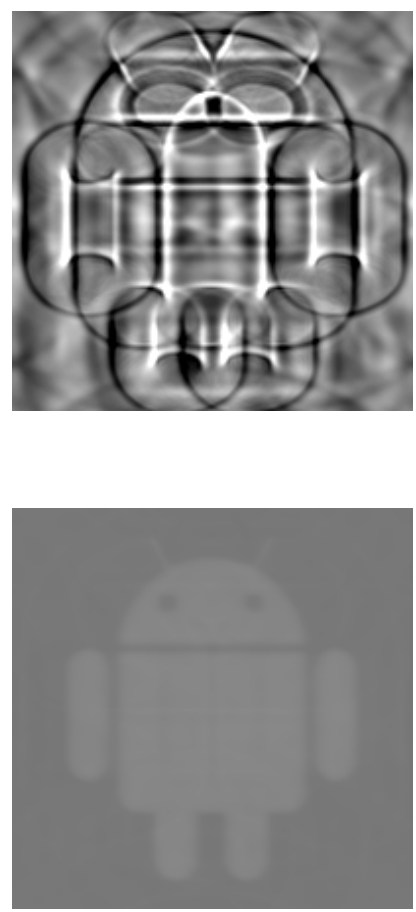

Figure 3.17: (a) Precompensated images and (b) simulated retinal images with contrast enhanced by the histogram side-clipping.

One simple and effective strategy to select thresholds is based on the relative frequency of the intensity bins, in comparison to the intensity with most occurrences, which is the intensity at the peak of the histogram. The search for low-side cutoff threshold starts from the intensity at the peak, moving towards lower levels (left of the peak) gradually, until an intensity with frequency less than a small percentage (e.g, $0.1 \%$ ) of the peak is found and assigned as $c_{L}$. The high-side cutoff threshold $c_{H}$ can be selected with a similar strategy, in which the search moves from the peak towards the right side. Obviously, larger contrast improvements can be achieved if the two thresholds $c_{L}$ and $c_{H}$ are selected closer to the peak. However, aggressive clipping of the histogram causes the elimination of more information in the compensated image, which may hurt the effect of precompensation. Figure 3.17 shows the precompensated images and simulated retinal images after the histogram 

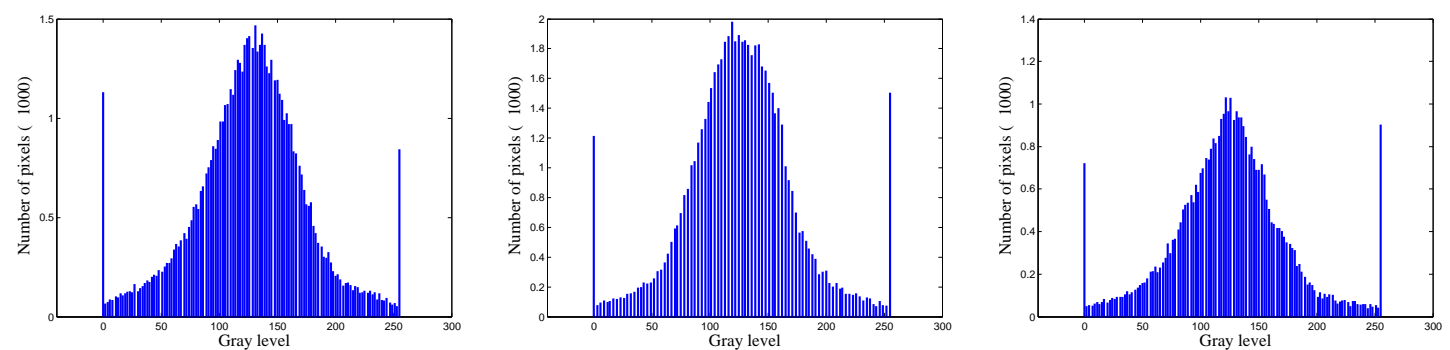

(a)
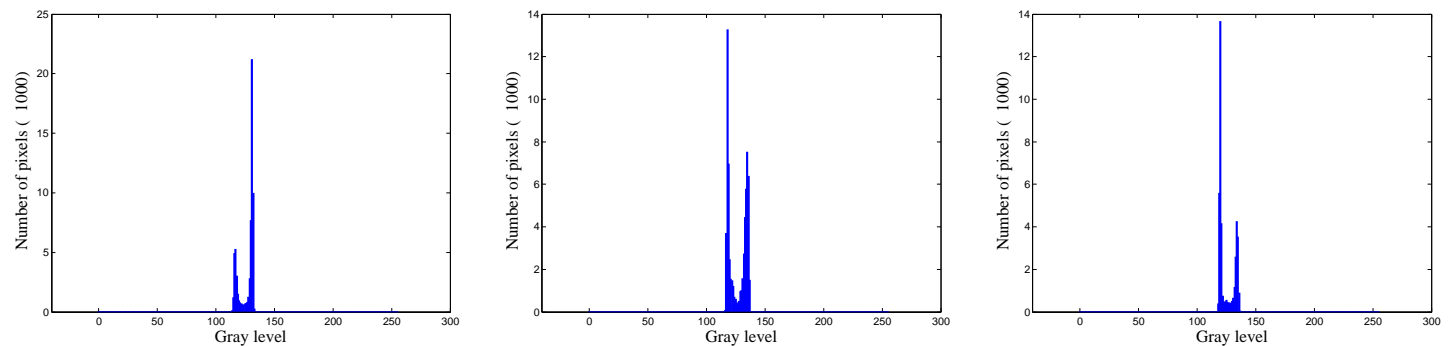

(b)

Figure 3.18: Histograms of the (a) precompensated images and (b) simulated retinal images, after the histogram side-clipping method is applied to expand the histograms of the precompensated images.

side-clipping method is applied, with histograms shown in Figure 3.18. Because the histogram side-clipping method extends the central histogram proportionally and the frequency of the intensities that have not been clipped are unchanged, the shape of the histogram is just expanded horizontally and therefore maintains most of the compensation pattern in the original compensated image. It is unavoidable that some compensation information is lost with the clipped intensities. However, the loss can be confined to an acceptable level, with careful selection of the thresholds.

\subsection{Dynamic Image Compensation}

The simulation results in last section are obtained under the assumption that the measured ocular aberrations are same as the ocular aberrations at the time of viewing. In reality, however, this perfect equality is not possible. Due to the dynamic 
properties of the eye, the variation of aberrations always exist and the difference between the measured aberrations and the aberrations at the time of viewing can be quite large in some cases.

\subsubsection{Dynamics of Aberration}

The optical characteristic of the human eye is not static and always exhibits temporal instability in the form of fluctuations caused by accommodation, which enables the eye to see objects clearly at different distances. Due to the accommodation fluctuations, the wavefront aberration of the eye and corresponding PSF also vary over time with dynamic features. The dynamics of accommodation has been intensively investigated in terms of lower order aberrations (defocus and astigmatism). Early studies $[8,9]$ indicated that the eye's defocus aberration constantly changes under steady viewing conditions. The magnitude of the accommodation response is approximately 0.03-0.5 Diopter with typical temporal frequencies less than $2 \mathrm{~Hz}[8]$.

More recently, the accommodation fluctuations of high order aberration were also explored, owing to the progress of wavefront sensing technology, especially the Hartmann-Shack wavefront sensor. It was reported that the fluctuations are also present in high order aberrations such as coma and spherical aberration $[25,30]$. Not surprisingly, the accommodation causes more fluctuations of aberrations when the pupil size is larger. In general, the variability of high order aberrations caused by accommodation has no significant impact on the retinal image quality. Moreover, because the accommodation fluctuations depend on many factors such as illumination conditions, pupil size and target vergence, it is not feasible to compensate them based on one single measurement under a particular circumstance. Another possible cause for the fluctuations of aberration is the eye movements, including pupil rota- 

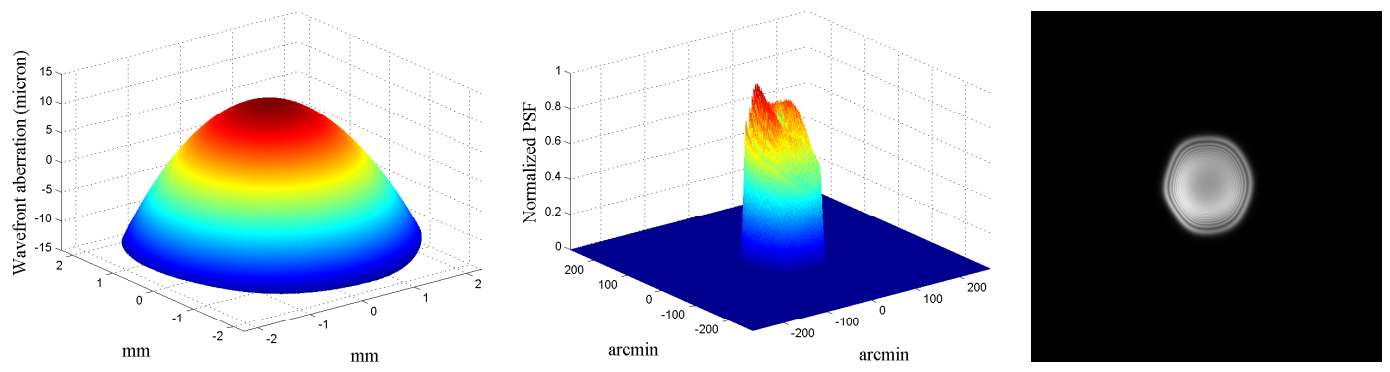

(a)
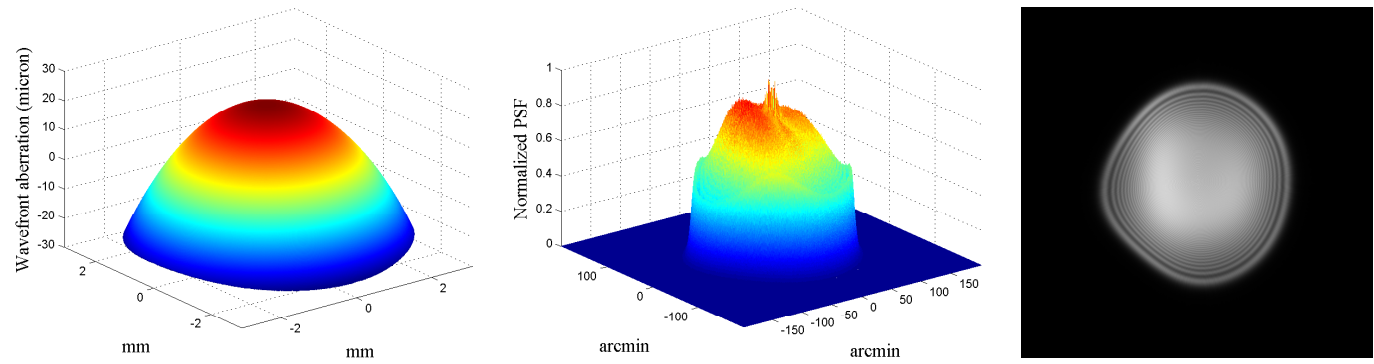

(b)
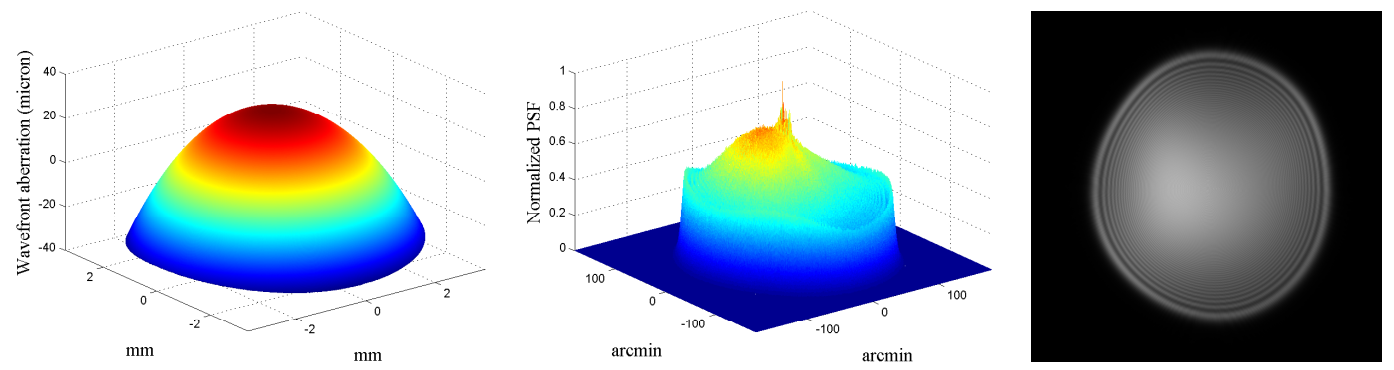

(c)

Figure 3.19: Comparison of measured ocular aberrations when the pupil diameter is (a) $4.5 \mathrm{~mm}$, (b) $6.0 \mathrm{~mm}$ and (c) $6.8 \mathrm{~mm}$, respectively. The ocular aberrations are measured from an eye with -8.6 D Spherical error and -0.6 D Cylindrical error with $85^{\circ}$ Axis. The PSFs with three different pupil sizes are also shown in the right column.

tion and translation, which are very small and unlikely to cause significant changes in the aberration [25]. 


\subsubsection{Effect of Pupil Size on Aberration}

Compared with the ocular aberration itself, the aberration fluctuations caused by accommodation and eye movements are relatively small. Usually, their effects on the retinal image are hard to notice and can be ignored. Therefore, these factors are not taken into account when we build the precompensation model. In our study, the most important factor that affects the ocular aberrations is the pupil size.

The pupil, which controls the light entering into the eye, is very sensitive to the intensity of the incident light. One well known fact is that the pupil dilates when the ambient becomes darker and constricts when the ambient becomes brighter. When we measure the wavefront aberration of the eye, the Zernike coefficients used to reconstruct the aberration are always reported with a pupil diameter or radius. If the aberration is reported without a pupil size, it is meaningless, since the Zernike coefficients are calculated over a normalized circle and have no size information. At different pupil sizes, the aberration of the same eye may exhibit large changes. Thus, the visual outcome can also be significantly varied if the pupil size changes. Figure 3.19 compares three frames of wavefront aberration of an aberrated eye (Spherical: -8.6 D, Cylindrical: -0.6 D, Axis: $85^{\circ}$ ), which were measured through a wavefront aberrometer under three different pupil diameters of $4.5 \mathrm{~mm}, 6.0 \mathrm{~mm}$ and $6.8 \mathrm{~mm}$, respectively. The differences of the pupil size are achieved by changing the illumination level at the time of measurement. The ocular aberration exhibits considerable increases as the pupil dilates, thereby generating a broader PSF. As shown in Figure 3.20, the overall RMS wavefront error of the ocular aberration is increased from $11.7 \mu m$ to $22.4 \mu m$ (a $91 \%$ increase) when the pupil diameter is enlarged from 4.5 $\mathrm{mm}$ to $6.0 \mathrm{~mm}$. When the pupil diameter is further enlarged to $6.8 \mathrm{~mm}$, there is another $31 \%$ increase, making the RMS error to be $29.4 \mu \mathrm{m}$. The high order RMS wavefront error is also increased from $0.8 \mu m$ to $1.6 \mu m(100 \%)$ when the pupil di- 


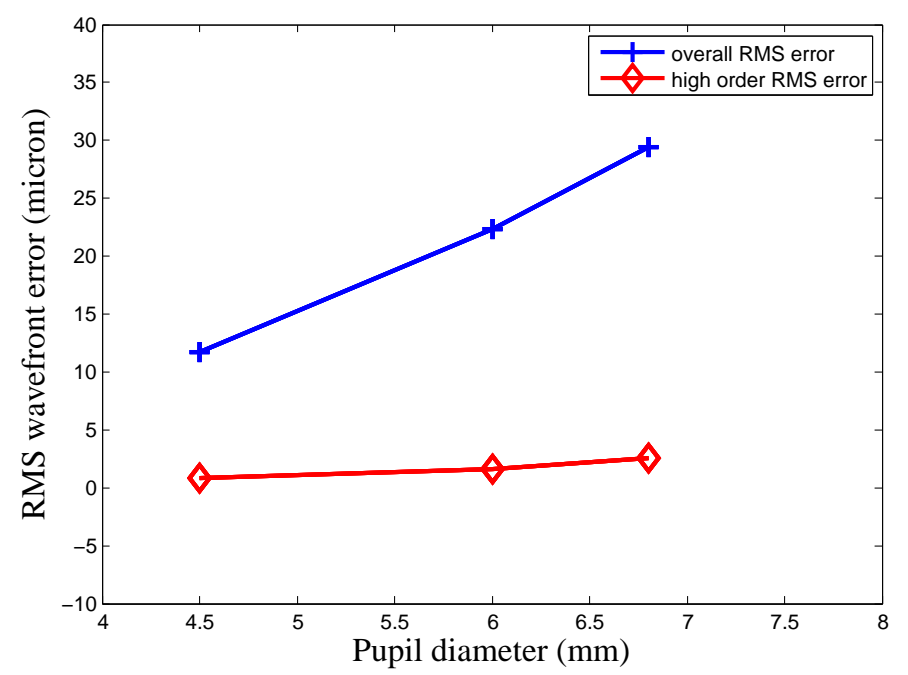

Figure 3.20: Overall wavefront RMS error and high order RMS error when the pupil diameter is $4.5 \mathrm{~mm}, 6.0 \mathrm{~mm}$ and $6.8 \mathrm{~mm}$, respectively.

ameter is enlarged from $4.5 \mathrm{~mm}$ to $6.0 \mathrm{~mm}$. When the pupil diameter is further enlarged to $6.8 \mathrm{~mm}$, the high order RMS wavefront error is increased to $1.6 \mu \mathrm{m}$ $(63 \%)$.

Therefore, static image compensation, in which the compensation model is constant and only determined by the measured aberration, is problematic. Considering that we measure the aberration under a dark environment and the computer is often used in a much brighter environment, the actual aberration at the time of viewing the image may greatly differs from the measured one. If we compensate the actual aberration using the mismatched compensation model derived form the measured aberration, the performance of precompensation method may be far from optimal.

\subsubsection{Aberration Resizing}

The pupil variations in realistic scenarios of computer use require us to update the ocular aberration used to produce the compensation. Thus, it is necessary to find a reliable and accurate method to resize the wavefront aberration measured with a 
specific pupil size to new sizes. The ocular aberration measured by wavefront sensors is usually reported as a set of Zernike coefficients. Due to the orthogonality of the Zernike polynomials, the Zernike coefficients are usually created after a particular pupil radius is set and normalized over a unit circle. For the same aberration error, the Zernike coefficients will be different if different pupil sizes are assigned. Thus, the problem becomes how to derive the new coefficients associated with a new pupil size from the original coefficients.

The Zernike polynomials are composed by three components: normalization component, radial component and azimuthal component. According to the ANSI standard for reporting ocular aberrations [87], it is preferable to report Zernike polynomials using a double indexing scheme as

$$
Z_{n}^{m}(r, \theta)=N_{n}^{m} R_{n}^{|m|}(r) M(m, \theta)
$$

where the index $n$ describes the order of the radial polynomials and the index $m$ describes the azimuthal frequency of the azimuthal components. The normalization component $N_{n}^{m}$ is defined as

$$
N_{n}^{m}=\sqrt{\frac{2(n+1)}{1+\delta_{m 0}}}
$$

where $\delta_{m 0}$ is the delta function that has the value of 1 when $m=0$ and 0 when $m \neq 0$. The radial function is defined as

$$
R_{n}^{|m|}(r)=\sum_{s=0}^{0.5(n-|m|)} \frac{(-1)^{s}(n-s) ! r^{n-2 s}}{s ![0.5(n+|m|)-s] ![0.5(n-|m|)-s] !}
$$

and the azimuthal function is defined as

$$
M(m, \theta)=\left\{\begin{array}{ccc}
\cos (m \theta) & \text { for } & m \geq 0 \\
-\sin (m \theta) & \text { for } & m<0
\end{array}\right.
$$

In the equations above, $n$ is the order of the Zernike polynomials. For a given $n, m$ can only take on values of

$$
-n,-n+2 \ldots n-2, n
$$


Consistent with Equation 3.1 in single indexing scheme, the wavefront aberration $W(r, \theta)$ can be represented by

$$
W(r, \theta)=\sum a_{n}^{m} Z_{n}^{m}(\rho, \theta)
$$

in double indexing scheme. One basic fact is that the wavefront aberration after pupil constriction is identical with the original one over the area confined by the smaller pupil size. Based on this equality, a couple of aberration resizing methods have been developed in analytical forms $[7,11,78]$. Since these methods resize the ocular aberration analytically and no resampling or interpolation operations are needed, the resized aberration has no error, theoretically.

In this study, the matrix transformation method proposed by Campell [7] is used to resize the measured aberration, for dynamically updating the image precompensation. Suppose the pupil radius is reduced from $P_{r}$ to $P_{r}^{\prime}$. Then, their ratio is given by

$$
\tau=P_{r}^{\prime} / P_{r}=\rho^{\prime} / \rho
$$

From the analysis above, it is known that

$$
\sum a_{n}^{m} Z_{n}^{m}(\rho, \theta)=\sum b_{n}^{m} Z_{n}^{m}\left(\rho^{\prime}, \theta\right)
$$

where $b_{n}^{m}$ is the new set of coefficients that describe the aberration after the pupil size is reduced. After expansion, the equation becomes

$$
\sum a_{n}^{m} N_{n}^{m} R_{n}^{|m|}(\rho) M(m, \theta)=\sum b_{n}^{m} N_{n}^{m} R_{n}^{|m|}\left(\rho^{\prime}\right) M(m, \theta) .
$$

The summation in Equation (3.44) can be represented in the form of multiplications of matrices and coefficient vectors. In order to obtain the analytical solution for $b$, the Zernike functions $Z_{n}^{m}$ and its components are reordered with a particularly 
designed sequence of double indexing pairs:

$$
\begin{gathered}
\left(-n_{\max }, n_{\max }\right),\left(-n_{\max }+1, n_{\max }-1\right),\left(-n_{\max }+2, n_{\max }-2\right) \\
\cdots \\
\left(n_{\max }-2, n_{\max }\right),\left(n_{\max }-1, n_{\max }-1\right),\left(n_{\max }, n_{\max }\right)
\end{gathered}
$$

where $n_{\max }$ is the highest order of the Zernike polynomials considered. Following the sequence, the terms of the Zernike functions $Z_{n}^{m}$ are decomposed into matrices $N, R$ and $M$ respectively. This sequence ensures that these matrices are inversable, facilitating the solution for the new coefficients.

Assuming the new coefficient vector $b$ can be obtained through a conversion matrix $C$, the relationship between the original coefficient vector $a$ and the new coefficient vector $b$ is simply shown as

$$
b=C a .
$$

Thus, the matrix representation of Equation (3.44) is given by

$$
T R M N a^{\prime}=R M N C^{\prime} a^{\prime},
$$

where $a^{\prime}$ is the vector of original coefficients with reordered sequence and $C^{\prime}$ is the conversion matrix for the reordered coefficients. As a diagonal matrix, $T$ is determined by the powers of the ratio $\tau$. $\mathrm{R}$ is the radial matrix with the terms of $R_{n}^{|m|}(\rho) . \quad M$ is the azimuthal matrix with the terms of $M(m, \theta)$ and $N$ is the normalization matrix with the terms of $N_{n}^{m}$. Thus, $C^{\prime}$ can be solved by

$$
C^{\prime}=N^{-1} R^{-1} T R N
$$

since the terms of $M$ are constants that can be canceled. Finally, in order to restore the coefficients to the original sequence, a permutation matrix $P$ is used to form the conversion matrix:

$$
C=P^{T} N^{-1} R^{-1} T R N P
$$


where $P^{T}$ is the transpose of $P$. When the conversion matrix $C$ is available, the new Zernike coefficients describe the aberration with the new pupil size can simply be obtained by Equation (3.45).

The matrix transformation method introduced above provides a convenient approach to obtain a new set of Zernike coefficients from a set of original coefficients. These new coefficients can be used to reconstruct the actual aberration, thereby updating the precompensation model in our study. It is important to indicate that the resizing of the ocular aberration is only valid when the new pupil size is smaller than the original. If the new pupil size is larger than the measured pupil size, the aberration data outside the original pupil area will not be available. Thus, there is no accurate and reliable way to resize the aberration to a larger pupil size with unknown information. Figure 3.21 shows an example in which one ocular aberration is resized to $50 \%$ of its measured pupil diameter.

\subsubsection{Comparison of Measured Aberration and Resized Aber- ration}

When we resize a measured ocular aberration to a new pupil size that is measured at the time of viewing, it is assumed that the resized aberration is approximately equal to the real aberration at the time of viewing, and this approximation is close enough that the aberration obtained by resizing can be used to replace the real aberration in updating the precompensation model. In order to verify this assumption, the resized aberrations of an eye with a specific pupil diameter, which are derived from measured aberrations with larger diameters, are compared to measured aberration with the same pupil diameter. The measured aberrations of the eye have been shown in Figure 3.19. In Figure 3.22, the measured aberrations with $6.8 \mathrm{~mm}$ pupil 

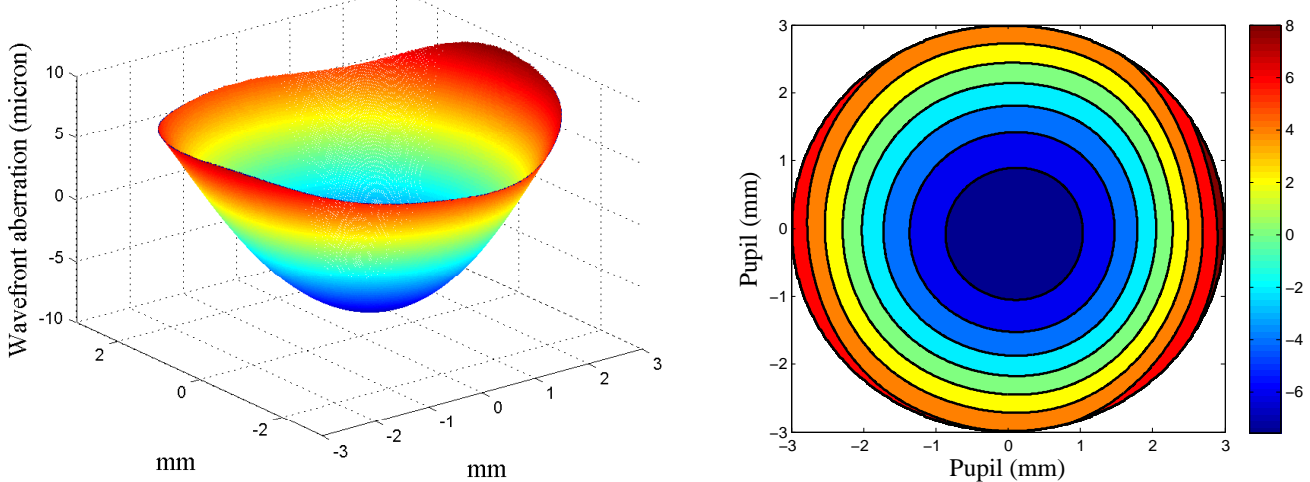

(a)
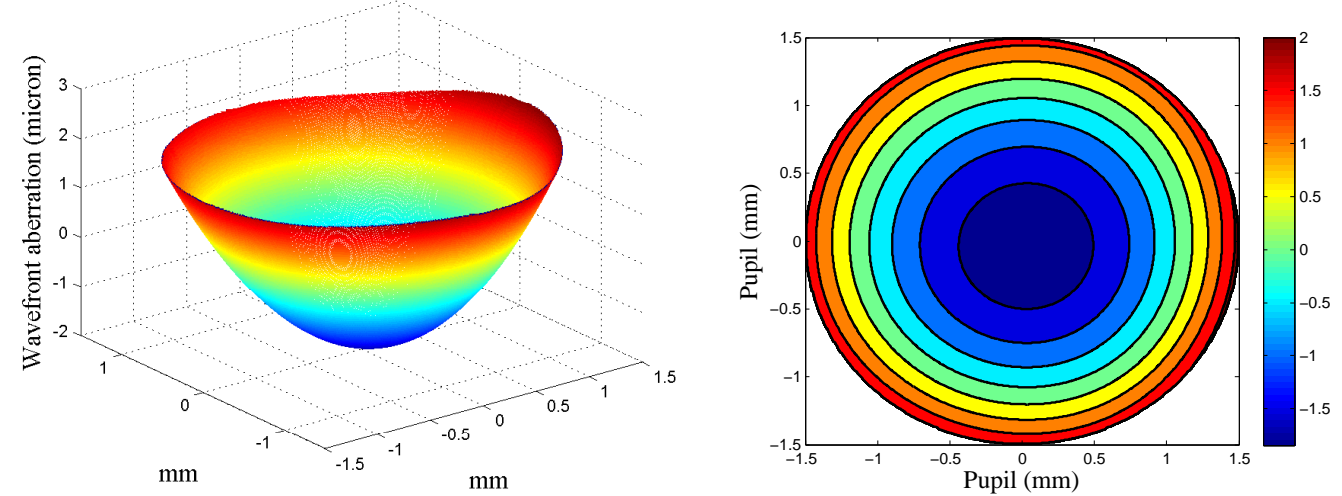

(b)

Figure 3.21: Example of ocular aberration resizing: (a) original ocular aberration, (b) resized ocular aberration.

diameter and $6.0 \mathrm{~mm}$ pupil diameter are resized to the aberration with $4.5 \mathrm{~mm}$ pupil diameter, respectively. These two resized aberration are compared with the measured aberration with $4.5 \mathrm{~mm}$ pupil diameter and the errors between them are investigated. It is found that both of the errors are quite small with the maxima of $0.91 \mu \mathrm{m}$ and $0.67 \mu \mathrm{m}$, respectively. Compared with the overall aberration, the error between the resized aberration and measured aberration is so small that can be ignored. 


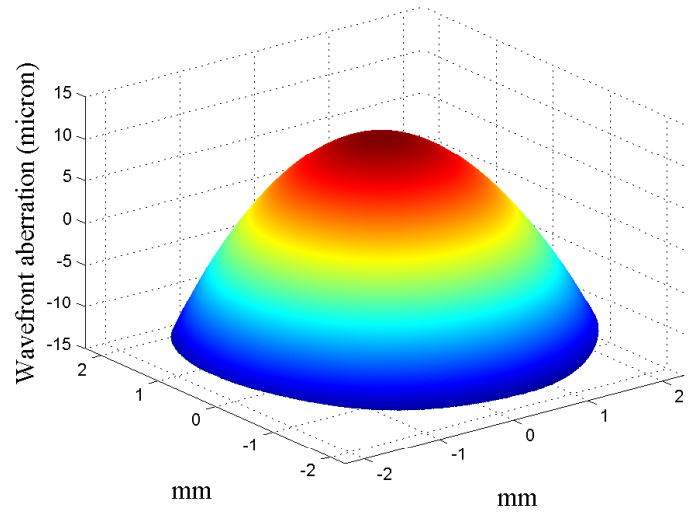

(a)

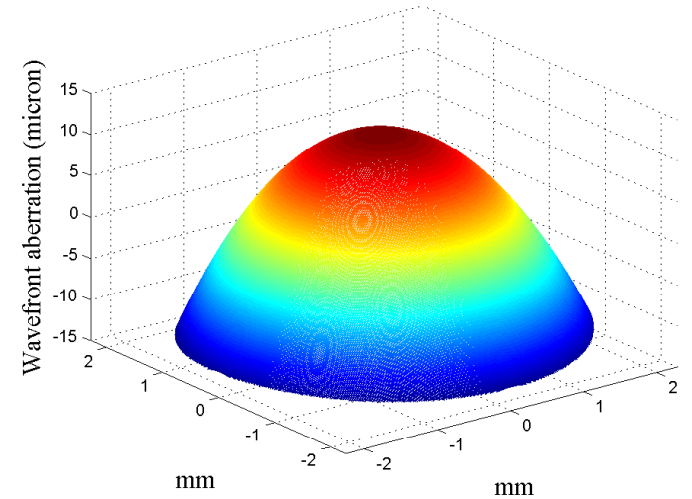

(b)

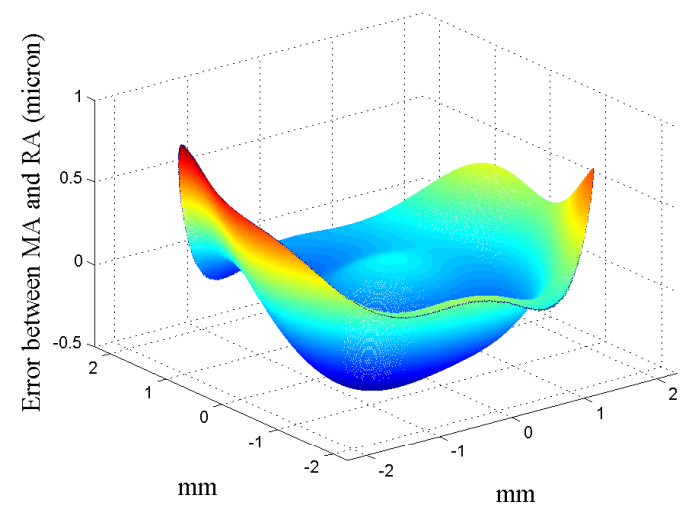

(d)

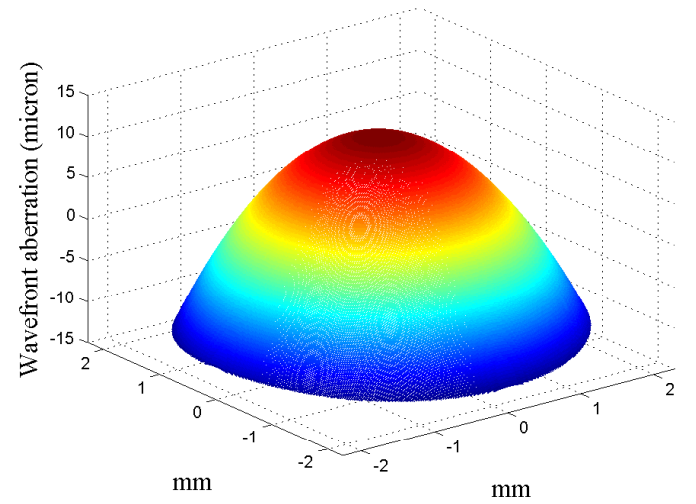

(c)

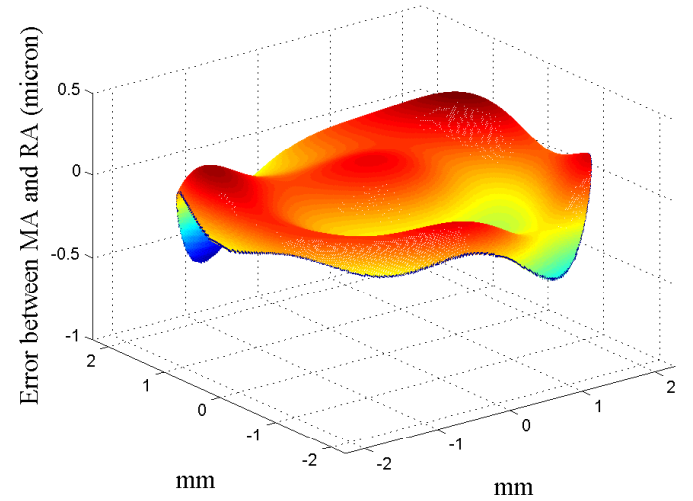

(e)

Figure 3.22: Comparison of resized aberration (RA) and measured aberration (MA) with the same $4.5 \mathrm{~mm}$ pupil diameter: (a) measured aberration, (b) resized aberration from $6.8 \mathrm{~mm}$ pupil diameter, (c) resized aberration from $6.0 \mathrm{~mm}$ pupil diameter, (d) error between the measured aberration and the resized aberration from $6.8 \mathrm{~mm}$ pupil diameter and (e) error between the measured aberration and the resized aberration from $6.0 \mathrm{~mm}$ pupil diameter. 


\subsubsection{Software Simulation}

If the image precompensation is not generated according to the actual aberration at the time of viewing but a mismatched aberration, the image blurring caused by the aberration will not be relieved. In extreme cases, the resulting retinal image may even have more severe distortion than the blurred image without any compensation. Figure 3.23 shows the simulation results when the precompensated images generated based on the aberration with $4.0 \mathrm{~mm}$ pupil diameter, are viewed by the same eye but with constricted pupil diameters of $3.2 \mathrm{~mm}, 3.5 \mathrm{~mm}$ and $3.8 \mathrm{~mm}$, respectively. This simulation is based on the same aberrated eye used in the simulations shown in Figure 3.13 and Figure 3.15.

It is found that, with mismatched compensation, the retinal images exhibit a strange distortion pattern different from the regular image blurring caused by the ocular aberration. When the pupil size at the time of viewing gets close to the pupil size with which the compensation is generated, this distortion tends to fade away, as shown in Figure 3.23(c). These simulation results confirm that the precompensation applied to images need to be updated and adjusted according to the real aberration data at the time of viewing.

If we resize the aberration to the same pupil diameter and update the image compensation according to it, the simulation results are demonstrated in Figure 3.24 , in which the precompensated images are adjusted according to the resized aberration with $3.2 \mathrm{~mm}$ and viewed by the eye with $3.2 \mathrm{~mm}$ pupil diameter. Not

surprisingly, the strange distortion patterns disappear and the shapes and edges of the retinal images become sharp and clear again. 

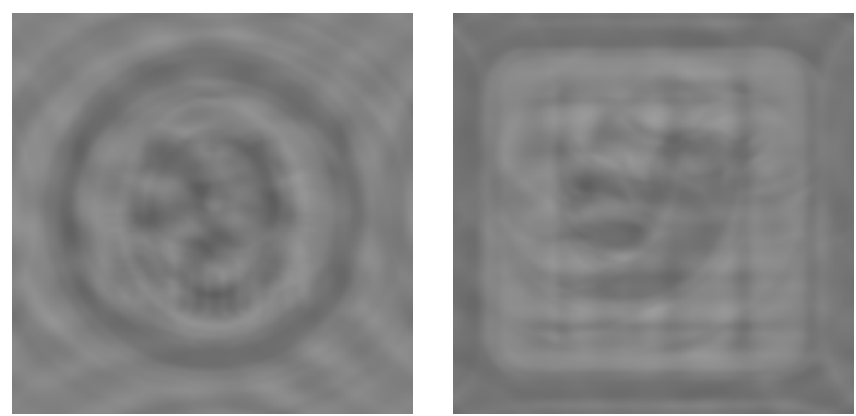

(a)
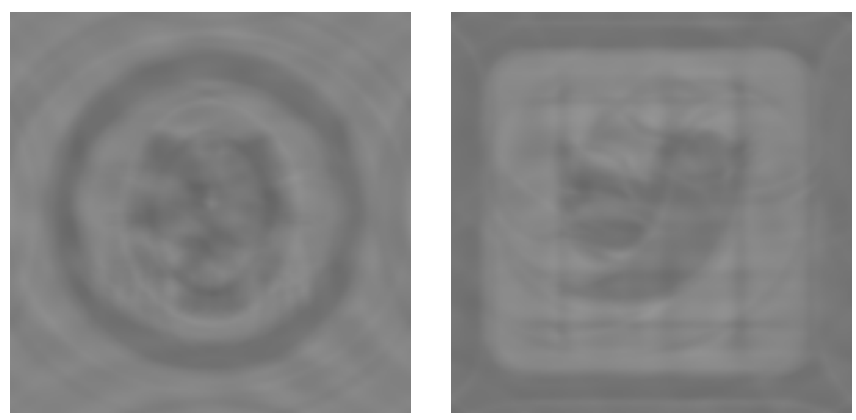

(b)
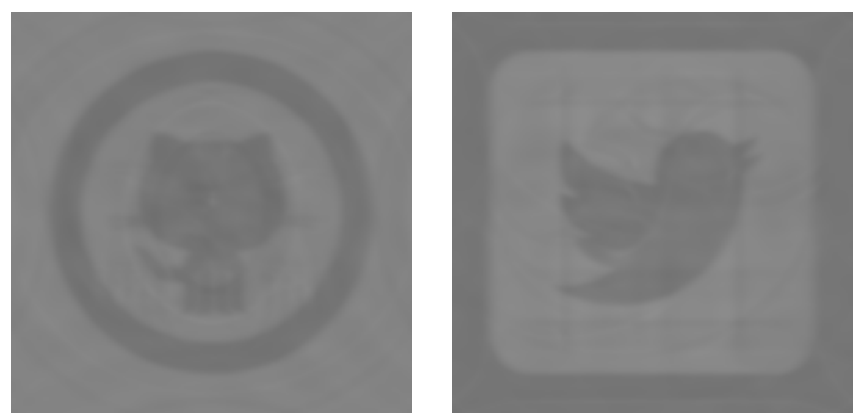

(c)
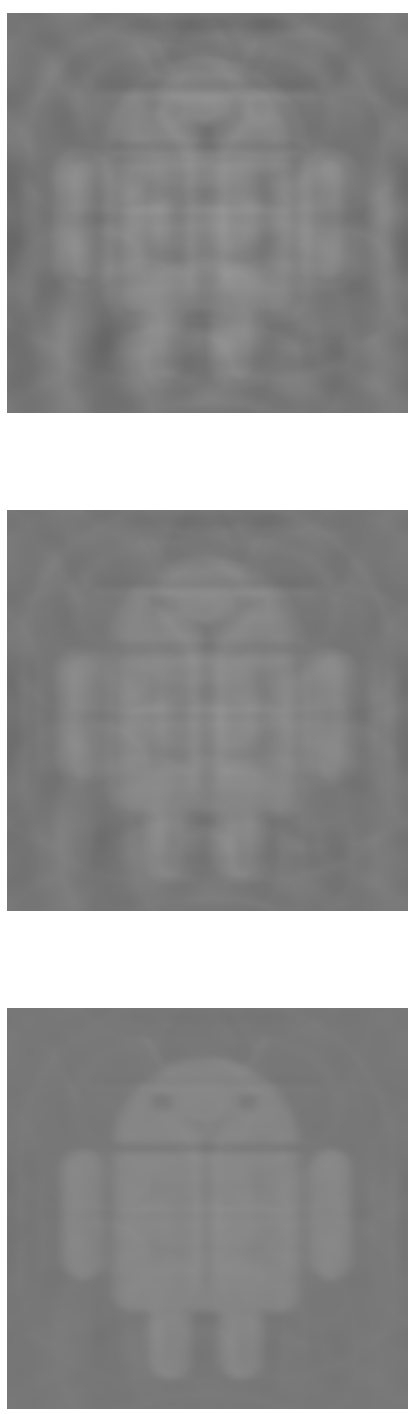

Figure 3.23: Simulated retinal images when the precompensated images generated based on the aberration with $4.0 \mathrm{~mm}$ pupil diameter are viewed by the same eye with constricted pupil diameter of (a) $3.2 \mathrm{~mm}$, (b) $3.5 \mathrm{~mm}$ and (c) $3.8 \mathrm{~mm}$, respectively.

\subsubsection{System Diagram}

So far, the potential of our image precompensation method on improving the visual performance of the computer user with ocular aberration, has been confirmed by the software simulation. To obtain the full benefit of the compensation, the compensation model must be updated based on the actual ocular aberration at the time of 

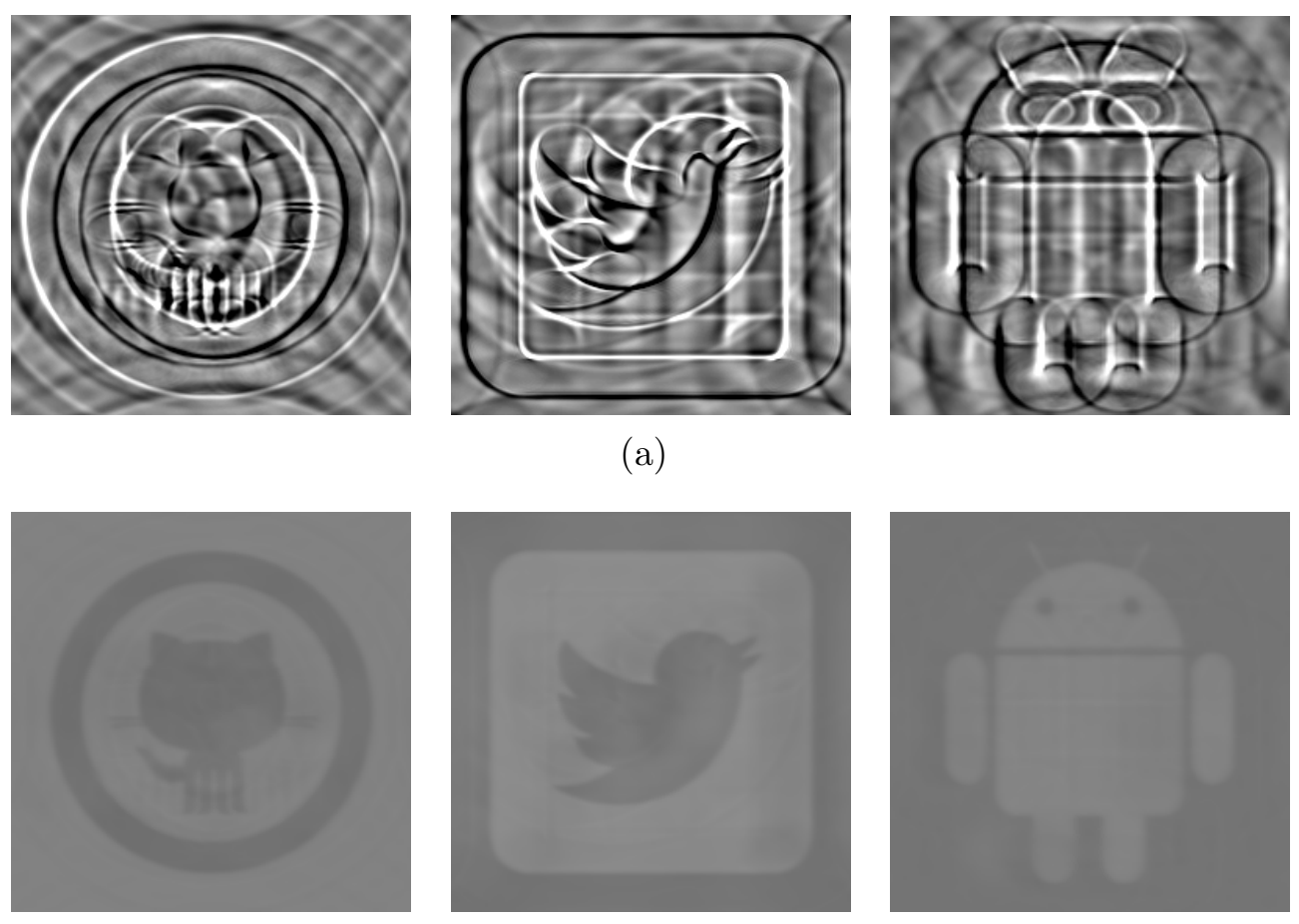

(a)
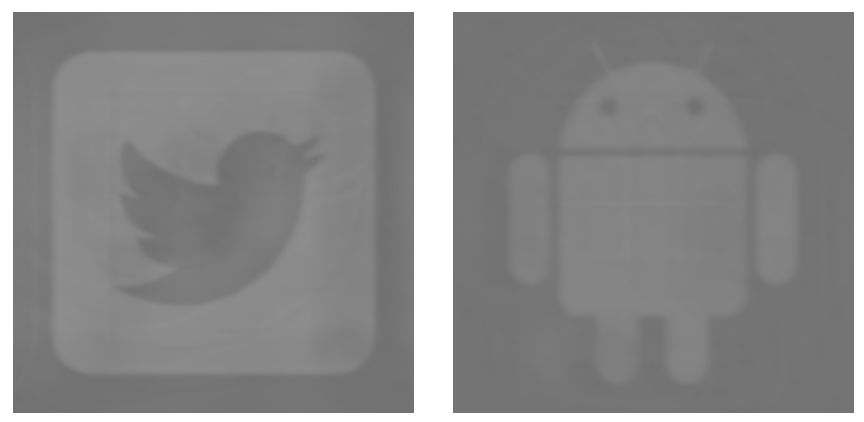

(b)

Figure 3.24: Simulated results when the precompensated images are updated according to the resized aberration with the matched pupil diameters at the time of viewing: (a) updated precompensated images and (b) corresponding retinal images.

viewing, in a dynamic way. As it is not feasible to measure the real-time aberration when the computer user views the computer screen, the resized aberration, which is derived from the original Zernike coefficients measured by the wavefront aberrometer, is used as the practical basis for the compensation update. By following this strategy, the implementation of our image precompensation system is greatly simplified, since the ocular aberration of one specific computer user only needs to be measured once. In practice, the measured Zernike coefficients can be stored in a personal file and imported into the system before using the computer. It is necessary to make sure that the measured aberration has larger pupil size than the actual pupil sizes at the time of viewing, so that the resized aberration is reliable. 


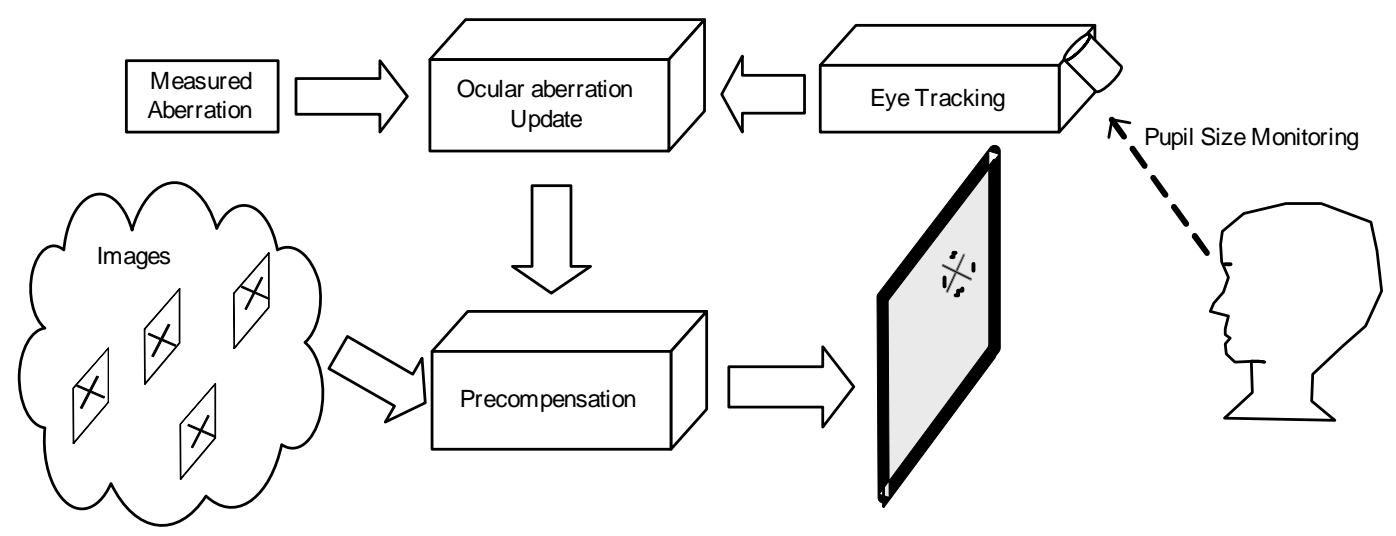

Figure 3.25: Schematic diagram of the dynamic image precompensation system.

In general, this is not a problem since the environment of normal computer use is usually brighter than the environment of wavefront measurement.

One the other hand, the real-time pupil diameters of the user are required to be available when the measured aberration is resized. The pupil variations can be monitored by using eye-tracking systems [13]. Typical eye-tracking systems measure the pupil size using a video camera and infrared illuminators, with the necessary image processing and analysis software. Most of the modern eye-tracking systems do not require head mounted equipment, providing accurate and unobtrusive pupil size measurement with frequencies from $30 \mathrm{~Hz}$ to $1000 \mathrm{~Hz}$. As the eye-tracking system is becoming common tool in Human Computer Interaction research [32], it can be easily integrated into our image precompensation system.

Figure 3.25 shows the schematic diagram of our dynamic image precompensation system. We can find that no special hardware is required except for the eye-tracking system and the system can be set up with any regular personal computer and display device. 


\subsection{Summary}

In this chapter, the modeling of retinal image formation of the computer user was introduced in detail, combined with some useful tools to describe the aberration of the eye including Zernike Polynomials, Point Spread Function (PSF), and Modulation Transfer Function (MTF). The retinal image formation can be described as a convolution process between the external image and the PSF of the eye. This convolution process can also be used to predict the visual outcome for the computer user when viewing images displayed on the screen. The effect of viewing distance on the defocus aberration was investigated and a corresponding adjustment method was given. We also introduced the PSF calibration criterion and methods according to the field of view (FOV) of the displayed image.

Aiming to improve the visual performance of the computer user, we introduced an image precompensation method in which the image blurring caused by the ocular aberration is expected to be counteracted by personalized image compensation. The performance of this method was evaluated through software simulations. In addition, a histogram side-clipping method was proposed to improve the contrast of the retinal image after precompensation.

As the ocular aberration used to build the compensation model varies with pupil size, the image compensation generated from static measured aberration may not match with the actual aberration at the time of viewing. In order to overcome this problem, we introduced a dynamic image precompensation strategy, in which the image compensation model is updated with real-time measurements of the pupil diameters. We also introduced the aberration resizing method that is used to update the compensation model. The effectiveness of this dynamic compensation method was validated through comparison of measured aberrations and corresponding re- 
sized aberrations, as well as through software simulation. Finally, we demonstrated the framework of the overall precompensation system, which will be used in the human subject evaluation experiment described in this dissertation later. 
CHAPTER 4

\section{ARTIFICIAL EYE EXPERIMENT}

The visual performance improvement achieved by the image precompensation method has been demonstrated through software simulation. The simulated image viewed by the computer user was obtained based on the theoretical imaging process, which is modeled as the convolution of the presented image and the PSF of the eye. To further verify the effectiveness of the precompensation method, we need to test it with real optical systems.

Before we apply the precompensation method to the human eye, we first test the method with an "artificial eye", which is a high-resolution digital camera. As the camera works in a similar way as the human eye, the performance of the precompensation method on the camera system can be considered as a valuable reference for the human eye tests later. More importantly, unlike the human eye, in which the image perception can only be evaluated subjectively, using of camera makes it possible for us to evaluate the image results in an objective manner, since the image formed by the camera is accessible.

\subsection{Experimental Instruments}

The "artificial eye" used in this experiment is a CMOS machine vision camera from PixeLink (PixeLink A781), providing monochrome images with resolution up to 6.6 megapixels $(2208 \times 3000)$. The camera is connected to personal computer through Firewire interface, with fast image transfer speed. In addition, the camera is equipped with an adjustable focusing barrel and an iris controller, as shown in Figure 4.1. 

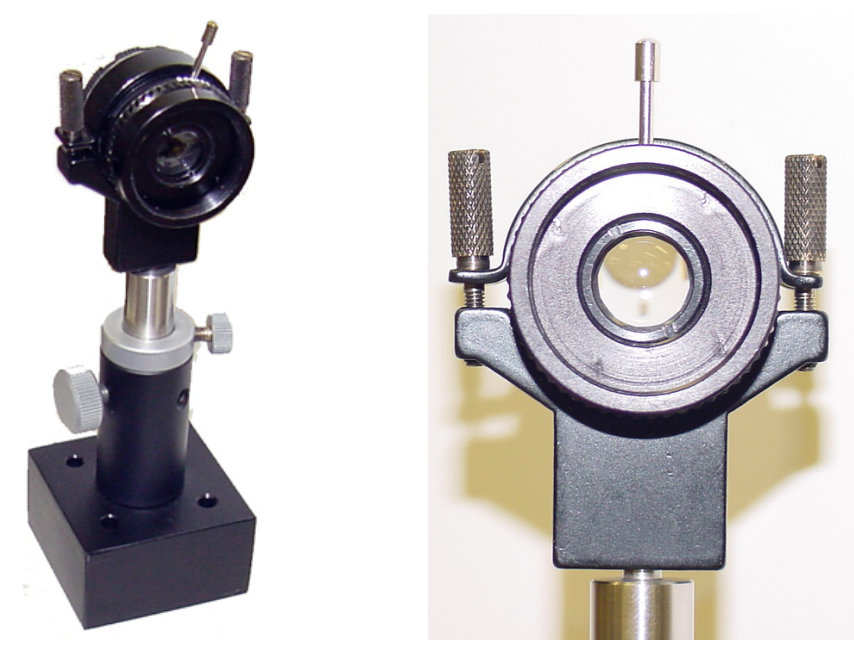

Figure 4.1: "Artificial eye" used in the experiment.

\section{YES ND}

Figure 4.2: Two $256 \times 256$ test images used in the artificial eye experiment.

The display device used in this experiment is a 24 inch flat panel LCD monitor (Dell G2410), with $1920 \times 1080$ resolution at $60 \mathrm{~Hz}$. The pixel pitch of the monitor is $0.277 \mathrm{~mm}$.

\subsection{Experimental Design}

Two $256 \times 256$ images, one containing the two words "YES" and "NO" and the other containing a "picture" icon, as shown in Figure 4.2, were selected as the test images presented on the screen. 

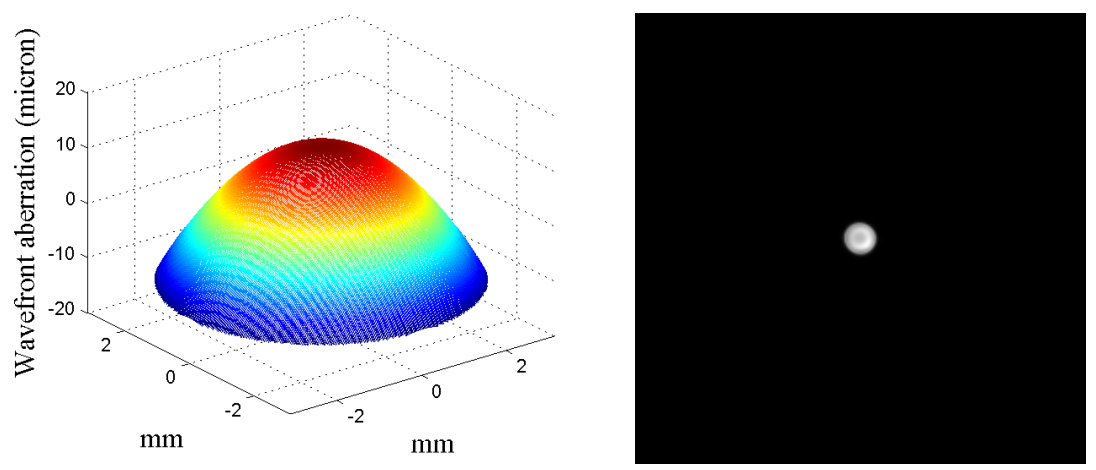

Figure 4.3: Wavefront aberration and calibrated PSF of the camera with $6.2 \mathrm{~mm}$ aperture diameter.

To simulate the aberration of the eye, a $-5 \mathrm{D}$ spherical error was produced by adjusting the focal length of the camera. Figure 4.3 shows the measured wavefront aberration and the calibrated PSF of the camera with $6.2 \mathrm{~mm}$ aperture diameter.

So that it would play the role of the viewer in the experiment, the camera was placed in front of the monitor and oriented to the displayed image at a viewing distance of $30 \mathrm{~cm}$. The original test images and precompensated images were presented on the screen in sequence, respectively. Further, in order to verify the necessity of the dynamic update to the compensation model, images with mismatched compensation and rematched compensation were also presented when the aperture diameter of the camera was constricted to $4.0 \mathrm{~mm}$. The aperture diameter of the camera was adjusted by manipulating the mechanical iris controller attached to the camera, as shown in Figure 4.1. In this experiment, the images viewed by the camera were captured with a size of $2208 \times 2208$. This was set through the region of interest (ROI) parameter of the camera. In addition, all images were captured with $0.06 \mathrm{~ms}$ exposure time and no gamma correction was applied. 

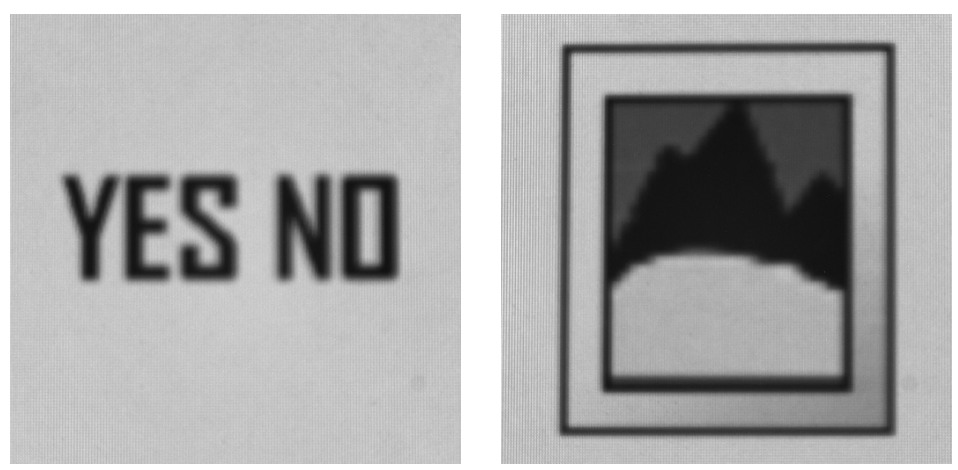

(a)
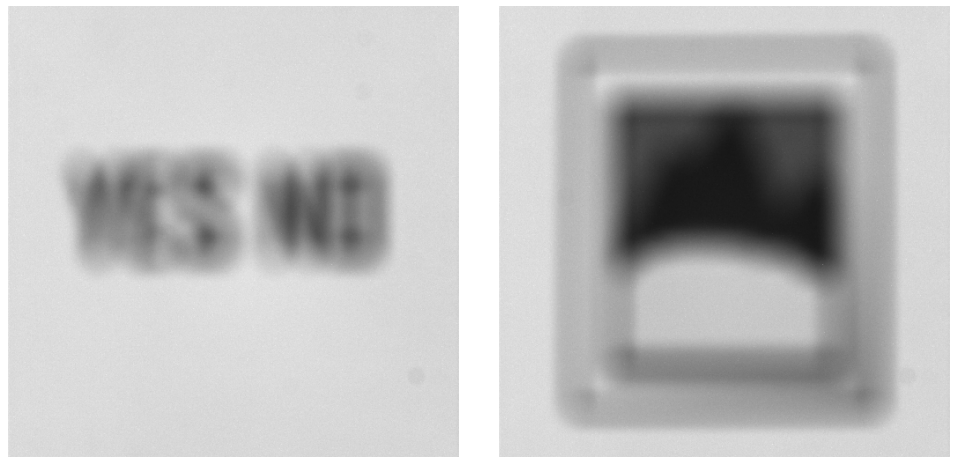

(b)

Figure 4.4: Images captured when the camera was (a) in focus and (b) defocused with -5 D spherical error.

\subsection{Results and Discussion}

Figure 4.4 shows the images captured when the aberrated camera was oriented to view the original test images. As a comparison, the figure also shows the images captured when the camera was correctly focused on the screen, that is, without any aberration being emulated. Obviously, due to the defocus aberration, the images captured by the aberrated camera were degraded with blurring. The precompensated images were generated according to the camera's wavefront aberration with $6.2 \mathrm{~mm}$ aperture diameter, as shown in Figure 4.5.

Figure 4.6(a) shows the images captured when the precompensated images were viewed by the aberrated camera with $6.2 \mathrm{~mm}$ aperture diameter. It is found, that, 

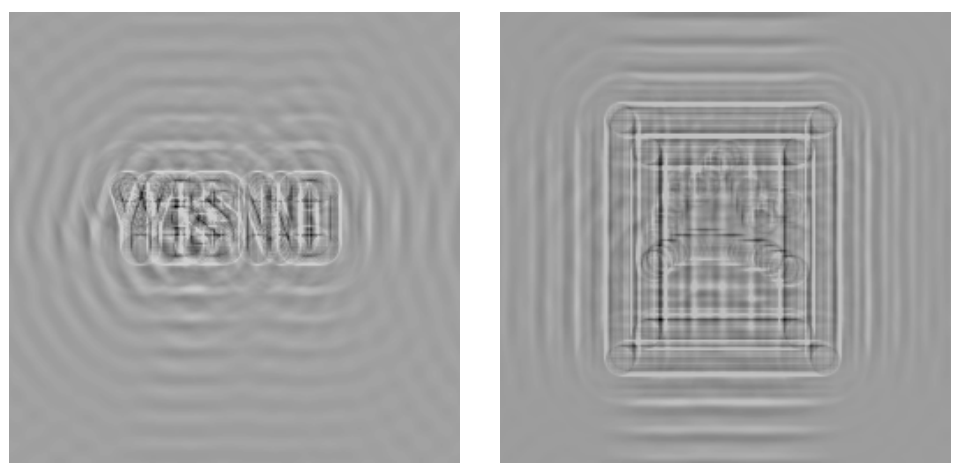

Figure 4.5: Precompensated images generated according to the camera's wavefront aberration with $6.2 \mathrm{~mm}$ aperture diameter.

with the precompensation, the images viewed by the aberrated camera exhibit more details and much sharper edges than the images captured when precompensation was not applied, even though the captured images also suffer problems of ringing artifacts and contrast loss, which are similar to the effects found in the software simulation.

Consistent with the simulation results, severe distortion was found in the images captured when the aberrated camera was targeted to view the images with mismatched precompensation, as shown in Figure 4.6(b). The mismatched precompensation here was caused by the reduction of aperture diameter form $6.2 \mathrm{~mm}$ to $4.0 \mathrm{~mm}$, which altered the wavefront aberration of the camera. As expected, the distortion caused by the mismatched precompensation disappeared, as shown in Figure 4.6(c), when the precompensation was updated according to the resized aberration of the camera.

\subsection{Summary}

In this chapter, we introduced implementation details and results of the "artificial eye" experiment, which was designed to test our image precompensation method. 


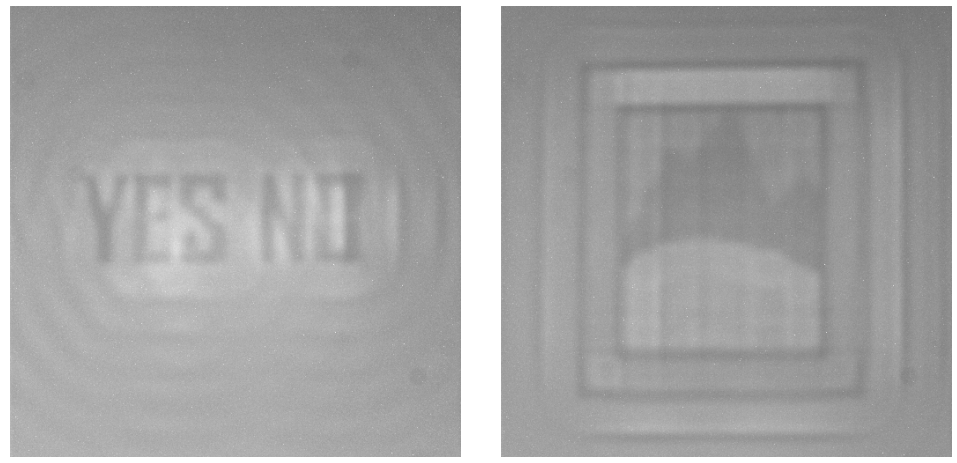

(a)

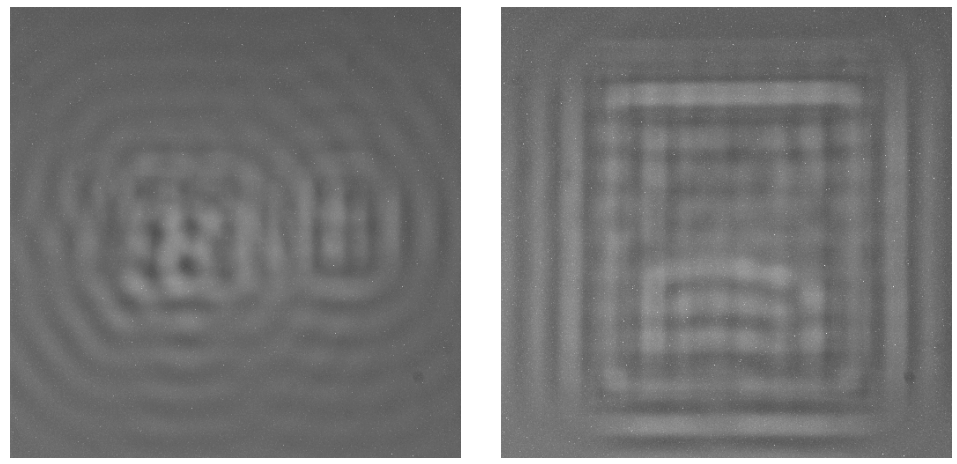

(b)
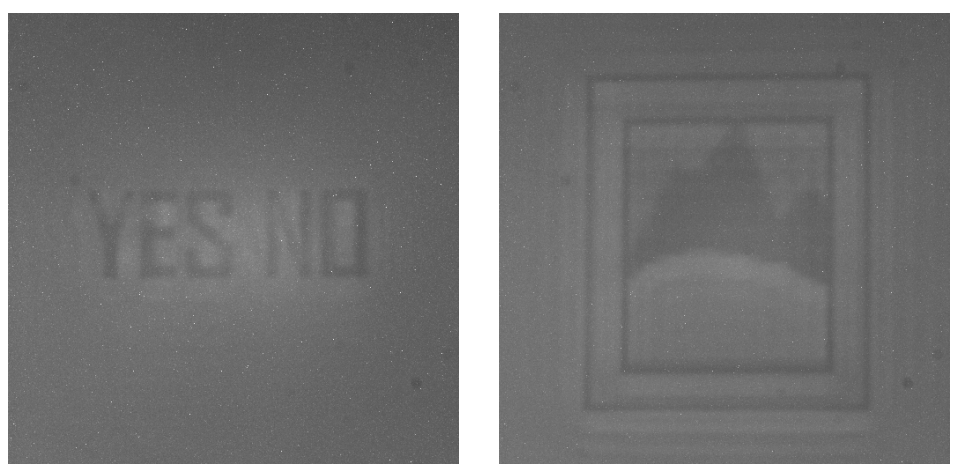

(c)

Figure 4.6: Results of the artificial eye experiment: (a) images captured when the precompensated images were viewed by the aberrated camera with $6.2 \mathrm{~mm}$ aperture diameter, (b)images captured when the precompensated images were viewed by the aberrated camera with $4.0 \mathrm{~mm}$ aperture diameter, (c) images captured when the images with updated precompensation were viewed by the aberrated camera with $4.0 \mathrm{~mm}$ aperture diameter. 
The results of this experiment are consistent with the simulation results in the previous chapter, thereby further confirming the effectiveness of the precompensation. 


\section{CHAPTER 5}

\section{HUMAN SUBJECT EXPERIMENT}

The primary goal of this study is to develop an image precompensation approach to overcome the image degradation caused by the aberration of the eye, thereby improving the visual performance of the computer user. In pervious chapters, the feasibility of this method has been verified through software simulation and its effectiveness has been confirmed through the experiment with an "artificial eye". This chapter describes an empirical experiment with human participants that was conducted in order to evaluate the visual benefit gained from the image precompensation method, under realistic viewing environment of computer using.

In the context of Human Computer Interaction (HCI), the recognition of graphical information, such as icons, pictures and menus, is usually the first step for many computer-based tasks. For those computer users with visual impairments, recognition of this graphical information is not always so straightforward, and sometimes can be hard to perceive correctly or efficiently. Thus, the ability to identify the presented graphical information can be considered as a reasonable metric to evaluate the visual performance of computer user with ocular aberration [31].

In this experiment, the effectiveness of the image compensation was verified through a series of image recognition tests. The accuracy in recognizing the original images without precompensation, images with static precompensation and images with dynamic precompensation, was recorded according to the responses of the observer, respectively, providing a quantitative way to evaluate the improvement on the visual performance. The recorded data was compared and investigated through statistical analysis. As a complement, we also collected the subjective assessment from the participants, in terms of their image perception during the tests. 


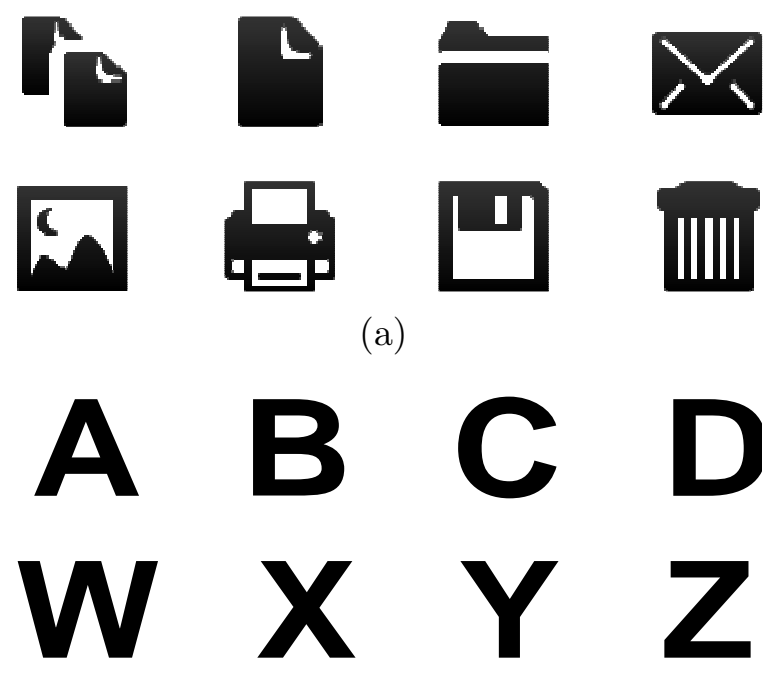

(b)

Figure 5.1: Test images used in the recognition tests: (a) eight icons that indicate Copy, Document, Folder, Email, Picture, Printer, Save and Delete, respectively, from left to right and up to bottom, (b) eight example letters (A, B, C, D, W, X, $\mathrm{Y}, \mathrm{Z})$ with Arial font. In each test condition, only 8 letters were randomly selected from the alphabet and presented.

\section{$5.1 \quad$ Experimental Images}

In this experiment, two types of images, icon and character, were presented on the screen as the targets to be recognized by the observer, as shown in Figure 5.1. The test images include eight icons, which are commonly used in various software interfaces, indicating Copy, Document, Folder, Email, Picture, Print, Save and Delete, respectively. In addition, images with 26 English letters in Arial font were also created, in order to investigate the performance of the image compensation on text. For consistency, only 8 letters were randomly selected from the alphabet and presented to the observer in each condition.

The test images have a size of $256 \times 256$ pixels. Each test image has two versions with different size of the content (letter or icon) embedded. The small size version spans $48 \times 48$ pixels and the large size version spans $72 \times 72$ pixels. Thus, 16 icon images and 52 letter images in total were created as the test images. All test images 
are black and white without any color information. The canvas of the test images was set to be completely white. When the image precompensation was disabled, the original image was displayed on the screen directly without any processing. Otherwise, the original image would be statically or dynamically precompensated according to the observer's ocular aberration and the resulting image would replace the original image to be presented.

\section{$5.2 \quad$ Subjects}

Twenty human subjects were recruited to participate in the experiment. The age of the subjects ranged from 20 to 33 years $(26.7 \pm 3.4)$. Thirteen of them were male and seven of them were female. Most of the subjects are undergraduate or graduate students at Florida International University. All of the subjects have high degree myopia or astigmatism, or both. The spherical error of the subjects ranges between $-3.24 \mathrm{D}$ and $-10.34 \mathrm{D}(-6.17 \pm 1.63 \mathrm{D})$ and the cylindrical error of the subjects ranges between $-0.22 \mathrm{D}$ and $-2.44 \mathrm{D}(-0.88 \pm 0.58 \mathrm{D})$. The detailed prescription of the subjects' eyes and the pupil diameters when their ocular aberrations were measured are shown in Table 5.1.

All of the subjects performed the image recognition tests with both eyes (one eye at a time). Thus, 40 eyes in total were tested in this experiment. An IRB approved consent form (FIU IRB Approval \# 062411-03) was signed by each subject before the test. During the time of the test, the subjects were not allowed to wear glasses or contact lenses. 
Table 5.1: Prescription of the subjects and pupil diameters when the ocular aberrations were measured.

\begin{tabular}{|c|c|c|c|c|c|}
\hline Subject & Eye & Sphere & Cylinder & Axis & Pupil Diameter \\
\hline 1 & OD & -4.84 & -0.22 & 177 & 6.4 \\
\hline 1 & OS & -4.30 & -0.57 & 11 & 5.8 \\
\hline 2 & OD & -5.58 & -0.36 & 24 & 5.7 \\
\hline 2 & OS & -4.55 & -0.58 & 147 & 5.4 \\
\hline 3 & OD & -5.15 & -0.65 & 8 & 5.6 \\
\hline 3 & OS & -5.80 & -0.58 & 174 & 5.9 \\
\hline 4 & OD & -4.31 & -0.25 & 124 & 3.5 \\
\hline 4 & OS & -3.24 & -0.90 & 167 & 3.3 \\
\hline 5 & OD & -10.34 & -0.94 & 4 & 4.7 \\
\hline 5 & OS & -10.09 & -0.95 & 169 & 4.3 \\
\hline 6 & OD & -6.17 & -0.82 & 4 & 6.2 \\
\hline 6 & OS & -6.20 & -1.26 & 171 & 6.0 \\
\hline 7 & OD & -5.37 & -0.88 & 8 & 6.3 \\
\hline 7 & OS & -5.81 & -0.56 & 174 & 6.1 \\
\hline 8 & OD & -6.85 & -0.23 & 167 & 6.5 \\
\hline 8 & OS & -6.97 & -0.32 & 175 & 6.1 \\
\hline 9 & OD & -6.20 & -2.03 & 7 & 4.8 \\
\hline 9 & OS & -6.15 & -1.87 & 178 & 4.5 \\
\hline 10 & OD & -5.15 & -0.53 & 173 & 3.8 \\
\hline 10 & OS & -4.96 & -1.17 & 173 & 3.8 \\
\hline 11 & OD & -4.27 & -1.30 & 3 & 4.9 \\
\hline 11 & OS & -4.72 & -1.75 & 175 & 4.8 \\
\hline 12 & OD & -5.86 & -1.45 & 179 & 6.8 \\
\hline 12 & OS & -5.10 & -2.44 & 169 & 7.1 \\
\hline 13 & OD & -5.53 & -1.16 & 177 & 5.7 \\
\hline 13 & OS & -5.01 & -1.78 & 8 & 5.9 \\
\hline 14 & OD & -7.53 & -0.44 & 167 & 5.1 \\
\hline 14 & OS & -7.53 & -0.23 & 113 & 5.1 \\
\hline 15 & OD & -6.35 & -1.40 & 4 & 6.5 \\
\hline 15 & OS & -5.82 & -1.86 & 2 & 5.0 \\
\hline 16 & OD & -5.10 & -1.33 & 174 & 5.6 \\
\hline 16 & OS & -3.49 & -2.06 & 176 & 5.2 \\
\hline 17 & OD & -6.39 & -0.70 & 118 & 6.2 \\
\hline 17 & OS & -7.42 & -0.38 & 53 & 6.1 \\
\hline 18 & OD & -6.87 & -0.16 & 25 & 6.0 \\
\hline 18 & OS & -5.45 & -0.32 & 151 & 5.7 \\
\hline 19 & OD & -7.86 & -0.36 & 163 & 5.3 \\
\hline 19 & OS & -7.32 & -0.54 & 6 & 5.6 \\
\hline 20 & OD & -5.55 & -0.99 & 167 & 5.8 \\
\hline 20 & OS & -5.25 & -0.94 & 9 & 6.1 \\
\hline
\end{tabular}




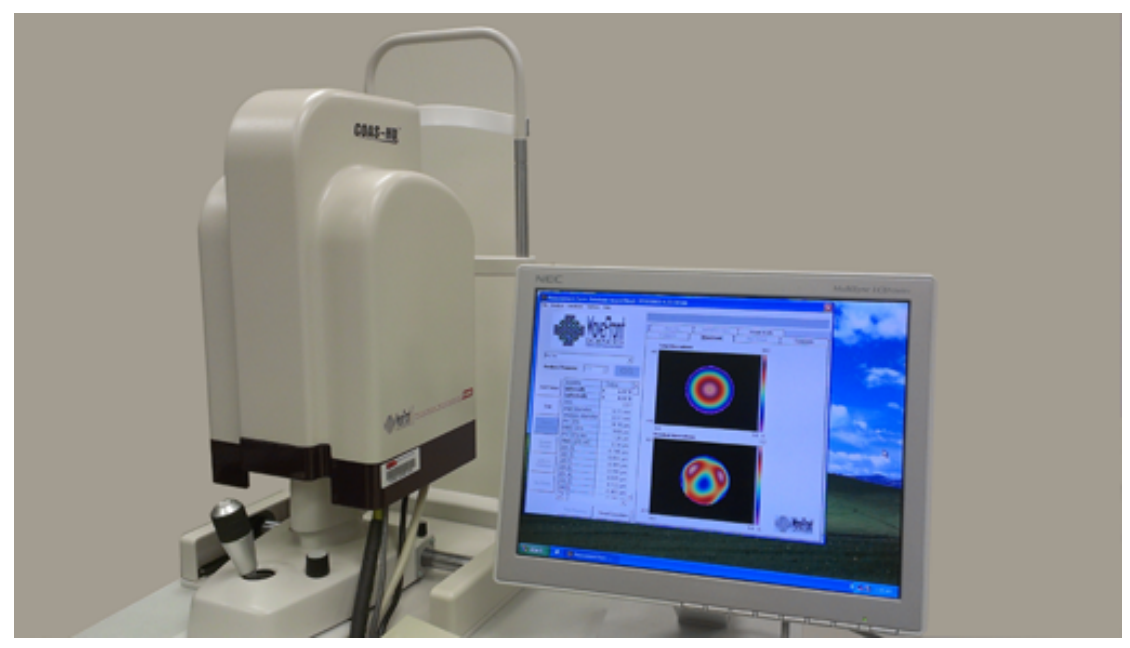

Figure 5.2: Complete Ophthalmic Analysis System (COAS-HD, Wavefront Sciences, Inc) that was used to measure the initial ocular aberration data of the subjects.

\subsection{Experimental Setup}

The experimental setup of the image recognition tests is consistent with the system diagram shown in Figure 3.25. In addition, user interfaces were designed to organize and control the presentation of the images to be recognized.

\subsubsection{Instruments}

The initial ocular aberration of each test eye was measured through a Complete Ophthalmic Analysis System (COAS-HD, Wavefront Sciences, Inc), as shown in Figure 5.2, which is based on the Hartmann-Shack wavefront sensing technology. The COAS-HD provides accurate and high definition measurement of wavefront aberration up to as high as 25 th order, with measurable Sphere range from -17 to 8 Diopters, and measurable Cylinder range from -5 to 5 Diopters. The system's measurable pupil diameter is as large as $9.5 \mathrm{~mm}$ [54]. In this study, we only used Zernike coefficients up to 6 th order that involve 28 terms of the Zernike polynomial. As the 


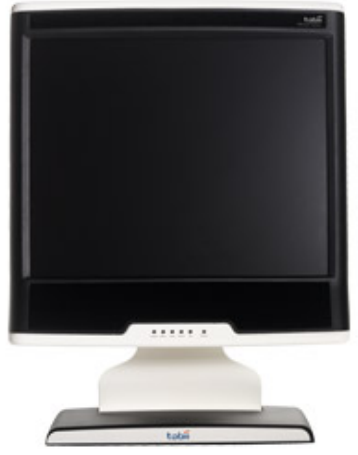

Figure 5.3: Tobii T60 eye-tracking system that was used to monitor the real-time pupil sizes of the subjects during the tests.

aberration data of each specific eye, the reported Zernike coefficients, along with the corresponding pupil diameter, were stored in a personal file after the measurement.

An eye tracking system (T60, Tobii, Inc), as shown in Figure 5.3, was used to monitor the real time pupil size of the subject. The T60 eye-tracking system provides unobtrusive and reliable pupil diameter measurement at a rate of $60 \mathrm{~Hz}$. The eye tracker is integrated in a 17 -inch TFT computer monitor, which was also used as the display device for presenting images in the experiment. The resolution of the monitor is $1280 \times 1024$. The tracking distance of the eye-tracking system is from $50 \mathrm{~cm}$ to $80 \mathrm{~cm}[53]$.

\subsubsection{User Interfaces}

In this experiment, two user interfaces were designed and developed to support the image recognition tests. At the beginning of the image recognition test, the subject was presented with a configuration interface, as shown in Figure 5.4. In this configuration interface, the required calibration of the T60 eye-tracking system was implemented. After we started the tracking system, the real-time pupil diameters of the subject's eyes were monitored and displayed in the interface. Besides, the 


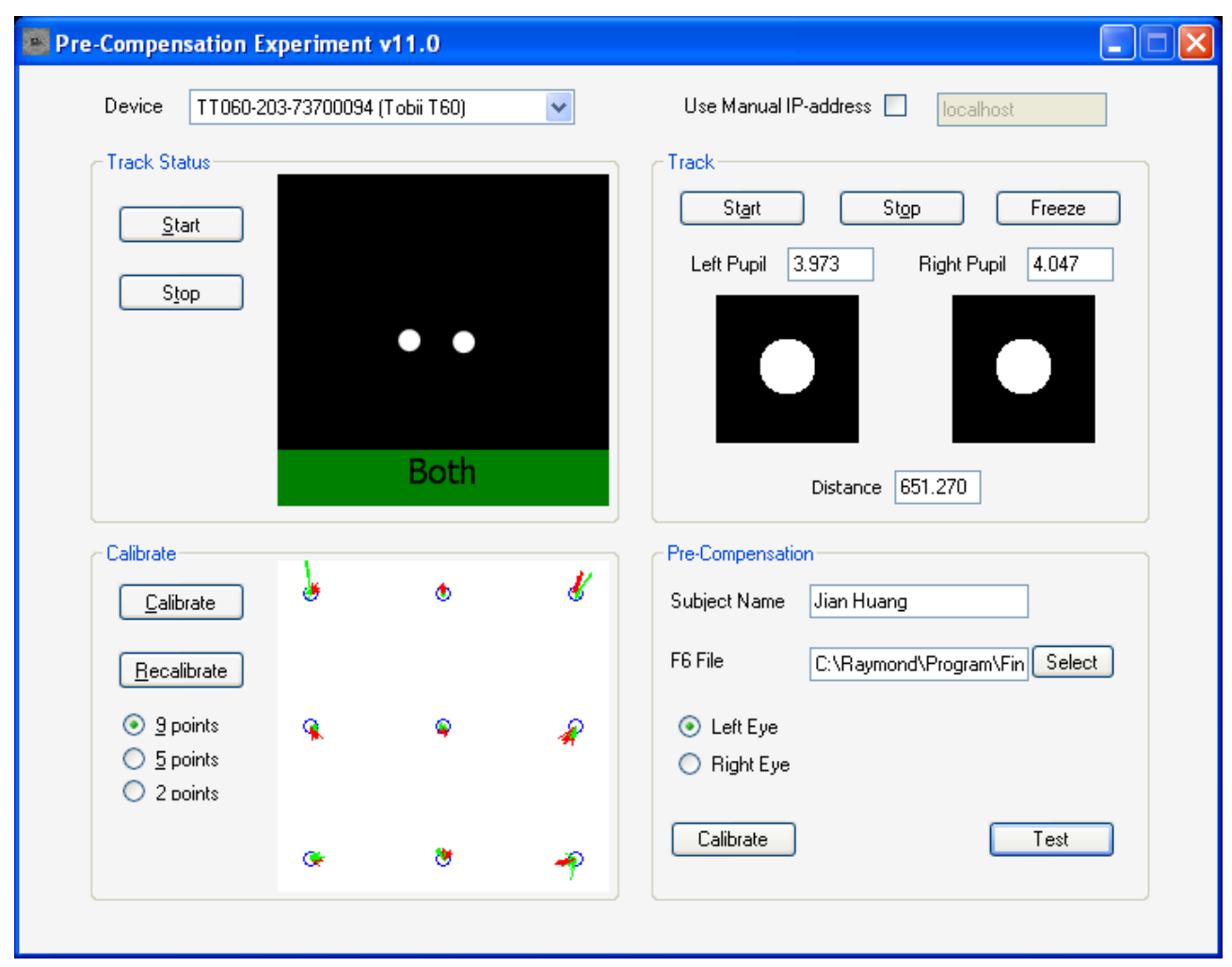

Figure 5.4: Configuration interface at the beginning of the image recognition test.

experimental record of the subject was established in the database and the test eye's initial aberration data was imported into the system.

The test interface of the experiment, as shown in Figure 5.5, has two major functions: controlling the presentation sequence of the test images and recording the recognition performance of the test eyes. During the tests, the experimental data were recorded based on the responses of the subjects and stored in a database for post analysis.

\subsection{Design and Procedures}

In order to study the effect of the dynamic precompensation on the image recognition ability of the subjects, the test images of icons and letters were presented with 


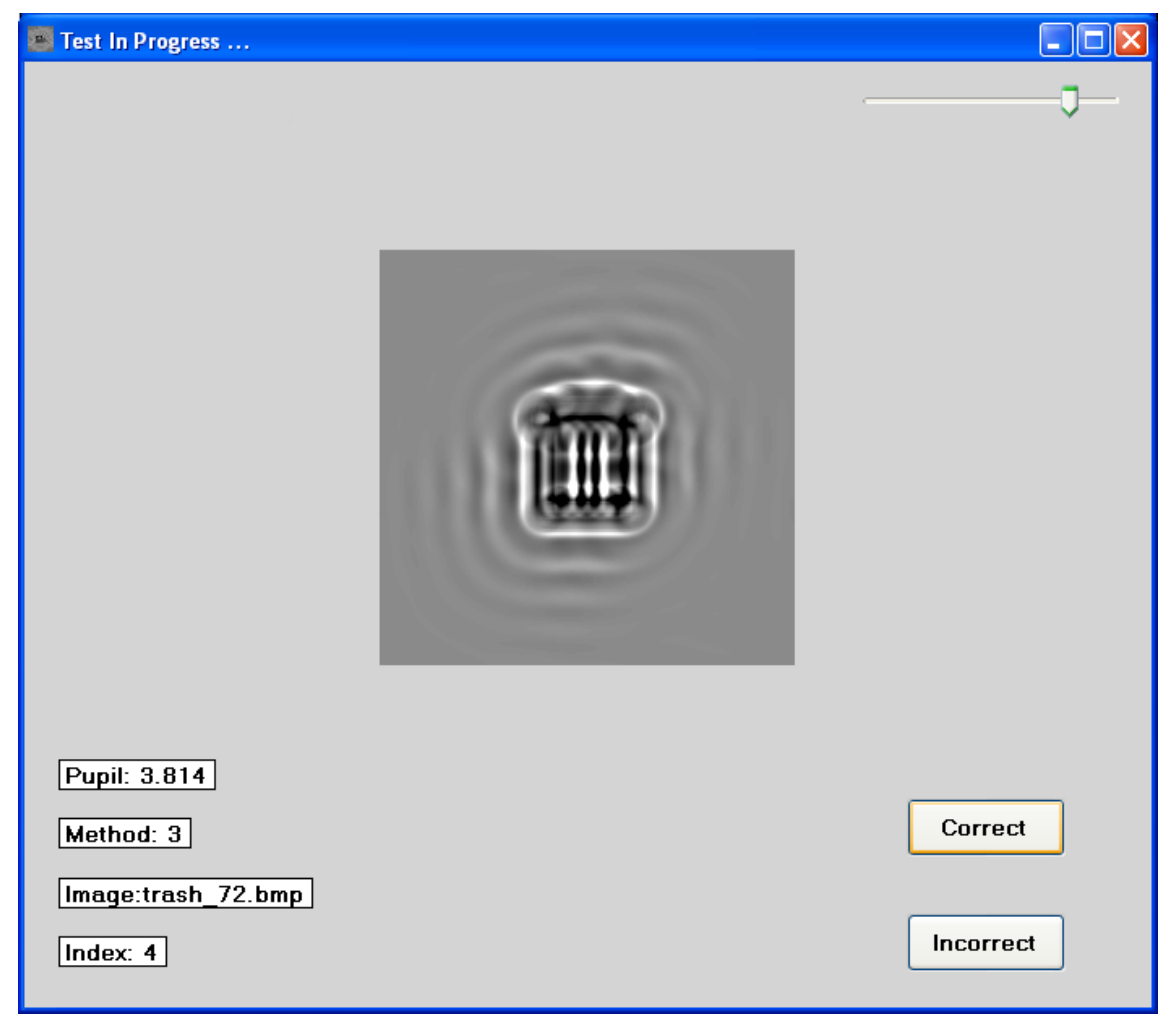

Figure 5.5: Test interface in the image recognition test.

static precompensation (SPC), with dynamic precompensation (DPC) and with no precompensation (NPC), respectively, in both the small and large sizes. Thus, the experiment used a factorial design with three factors: image category, with two levels (ICON or LETTER), content size, with two levels (SMALL and LARGE) and image precompensation method, with three levels (SPC, DPC, and NPC). The dependent variable was the number of correct recognitions made by the subjects in each condition, ranging from 0 to 8.

Before the image recognition test, the ocular aberrations of the two eyes of the subject were measured by the COAS-HD. In order to dilate the pupil of the eyes, the measurement was conducted under dark illumination environment. The measured aberration data was imported as the reference for image compensation at the beginning of image recognition tests. Since the aberrations in each eye of an 
individual are generally different, the recognition tests in this experiment were based on the monocular vision of the subjects. Likewise, the image recognition accuracies with different precompensation methods were evaluated by eye and not by subject. During the test, one of the two eyes of the subject would be covered by an eye patch while the other one was viewing the images presented. The image recognition tests were performed under office lighting condition, thereby ensuring that the pupil sizes of the subjects during the tests were larger than the initial ones measured with Zernike coefficients, using the COAS-HD.

\subsubsection{Image Recognition Test}

During the image recognition tests, the subjects were instructed to sit in front of the computer monitor at a distance of 25 inches $(64 \mathrm{~cm})$. The test images were presented to the subject one by one. The presentation of the images was grouped by the treatment combination of content size, image category and precompensation

method, according to the factorial design of the experiment. Within each group, the presentation sequence of test images was randomized. In addition, the order of groups to be presented was also randomized.

Within each group, each test image selected was presented only once, with SPC, DPC or NPC. As 8 test images were presented in each group, there were

$$
8 \times 2(\text { category }) \times 2(\text { size }) \times 3(\text { method })=96
$$

trials in the test of each eye. In each trial, the subject was asked to identify the presented image verbally. Based on the answer, the recognition trial was recorded as correct or incorrect. The test images were presented on the screen for only 3 seconds. After that, the current test image would be hidden. This also helped to promote the spontaneity of the subject's answers and prevent the subjects from 
fatigue. The image recognition test for each eye lasted for around 20 minutes. After completing the test of one eye, the subject was allowed to take a 5 minute break before doing the test of the other eye.

\subsubsection{Subjective Assessment}

In order to investigate the visual perception of the subject to the images generated with different precompensation methods, a brief questionnaire was conducted immediately after the completion of the image recognition tests. The questionnaire in this experiment includes four questions, as shown in Table 5.2. Based on how they felt about the images they viewed during the test, the subjects were required to choose the answers from the options. The images presented with precompensation were easily distinguished by the subjects from the original ones without precompensation, because of their darker background. During the tests, before moving to the next condition with a different precompensation method, the subject would be notified in advance. In practice, when we conducted the questionnaire, the three precompensation methods were indexed as method \#1, method \#2 and method \#3 according to the presenting order, facilitating the subject's comparison and assessment.

\subsection{Results and Discussion}

\subsubsection{Statistical Description}

Table 5.3 shows the twelve different conditions of the image recognition test, which are obtained from the combinations of three within-subjects factors. Under each condition, 8 recognition trials were performed by each test eye. The mean and standard deviation of the number of correct recognitions made by each test eye 
Table 5.2: Questionnaire conducted after the completion of the image recognition test.

\begin{tabular}{|c|c|c|}
\hline & Questions & Options \\
\hline Q1 & $\begin{array}{l}\text { Was the sharpness of DPC images better, } \\
\text { equivalent, or worse than NPC images? }\end{array}$ & $\begin{array}{l}\text { (1) better } \\
\text { (2) equivalent } \\
\text { (3) worse }\end{array}$ \\
\hline Q2 & $\begin{array}{l}\text { Was the sharpness of DPC images better, } \\
\text { equivalent, or worse than SPC images? }\end{array}$ & $\begin{array}{l}\text { (1) better } \\
\text { (2) equivalent } \\
\text { (3) worse }\end{array}$ \\
\hline Q3 & $\begin{array}{l}\text { Which image category was more easily } \\
\text { recognized after compensation? }\end{array}$ & $\begin{array}{l}\text { (1) icon } \\
\text { (2) letter } \\
\text { (3) same }\end{array}$ \\
\hline Q4 & $\begin{array}{l}\text { Which problem of precompensation } \\
\text { impede your recognition most? }\end{array}$ & $\begin{array}{l}\text { (1) low contrast } \\
\text { (2) ringing effect } \\
\text { (3) other }\end{array}$ \\
\hline
\end{tabular}

under different conditions are tabulated in Table 5.4., It was encouraging to find that the average correct recognitions exhibited considerable increase, in all of the four groups of test images (small letter, small icon, large letter and large icon), when dynamic precompensation was used. Overall, with the application of dynamic image precompensation to the test images, the recognition accuracy of the subjects improved from $48.2 \%(3.86 / 8)$ and $40 \%(3.2 / 8)$ to $73.5 \%(5.88 / 8)$, comparing to images with no precompensation and static precompensation, respectively. This improvement of recognition accuracy is revealed more directly in Figure 5.6, in which the number of correct recognitions performed under different precompensation methods are compared in parallel.

\subsubsection{Results of ANOVA}

As the same subjects took part in all conditions of the image recognition tests, the experimental data was analyzed through a three-way Analysis of Variance (ANOVA) 
Table 5.3: Twelve different conditions in the image recognition tests.

\begin{tabular}{cccr}
\hline Content Size & Image Category & Compensation Method & Condition \\
\hline small & letter & NPC & small letter with NPC \\
small & letter & SPC & small letter with SPC \\
small & letter & DPC & small letter with DPC \\
small & icon & NPC & small icon with NPC \\
small & icon & SPC & small icon with SPC \\
small & icon & DPC & small icon with DPC \\
large & letter & NPC & large letter with NPC \\
large & letter & SPC & large letter with SPC \\
large & letter & DPC & large letter with DPC \\
large & icon & NPC & large icon with NPC \\
large & icon & SPC & large icon with SPC \\
large & icon & DPC & large icon with DPC \\
\hline
\end{tabular}

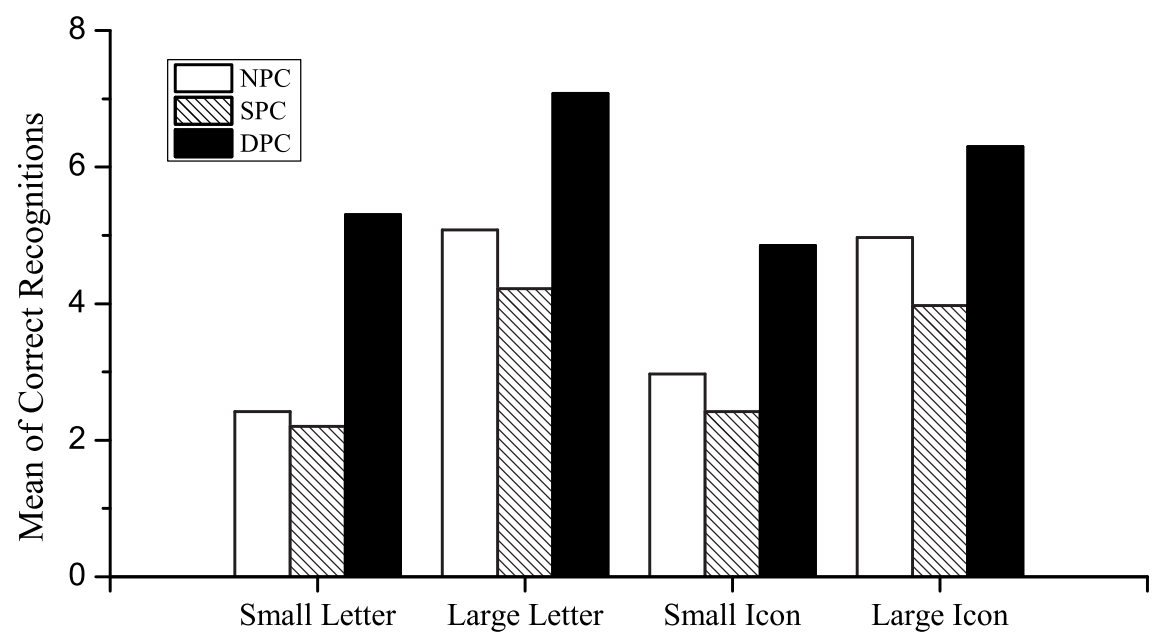

Figure 5.6: Bar graph of average correct recognitions under different conditions, in which the recognition performances with NPC, SPC and DPC are compared in parallel.

with repeated measures, for evaluating the significance of the benefit from our dynamic precompensation method. The effects of three within-subjects factors (image category, content size and precompensation method) were investigated based on the number of correct recognitions made by the subjects, in each condition of the tests. 
Table 5.4: Descriptive Statistics of the image recognition tests under different conditions.

\begin{tabular}{lccc}
\hline Condition & Mean & Std. Deviation & $\mathrm{N}$ \\
\hline small letter with NPC & 2.42 & 2.049 & 40 \\
small letter with SPC & 2.2 & 2.289 & 40 \\
small letter with DPC & 5.3 & 1.698 & 40 \\
small icon with NPC & 2.97 & 2.13 & 40 \\
small icon with SPC & 2.42 & 1.567 & 40 \\
small icon with DPC & 4.85 & 1.791 & 40 \\
large letter with NPC & 5.08 & 2.358 & 40 \\
large letter with SPC & 4.22 & 2.224 & 40 \\
large letter with DPC & 7.08 & 1.118 & 40 \\
large icon with NPC & 4.97 & 2.348 & 40 \\
large icon with SPC & 3.97 & 1.804 & 40 \\
large icon with DPC & 6.3 & 1.556 & 40 \\
\hline
\end{tabular}

In contrast of the need of the assumption of normality in tests where the experimental data is independent between subjects, it is the sphericity of data that needs to be met in repeated measures, in which the data comes from the same group of subjects and is recorded under different experimental conditions. Sphericity requires that the variances of the differences between experimental factors are equal [15]. Thus, before we applied the ANOVA, the assumption of sphericity of the experimental data was inspected using Mauchly's test, as shown in Table 5.5. From the results of Mauchly's test, it was found that the assumption of sphericity was not met between the levels of precompensation methods, $p<0.05$. The sphericity was also violated by the interaction effect between the factors of image category and precompensation method. Because the assumption of sphericity is not met, the probability of Type II error will be increased in the analysis and the F-ratio can not be trusted directly. In this study, the violation of sphericity was corrected by the 
Table 5.5: Results of Mauchly's sphericity test to the experimental data.

\begin{tabular}{lccrr}
\hline Within Subjects Effect & Mauchly's W & Chi-Square Approximation & df & Sig. \\
\hline Size & 1 & 0 & 0 &. \\
Category & 1 & 0 & 0 &. \\
Method & 0.853 & 6.042 & 2 & 0.049 \\
Size*Category & 1 & 0 & 0 &. \\
Size*Method & 0.942 & 2.272 & 2 & 0.321 \\
Category*Method & 0.809 & 8.049 & 2 & 0.018 \\
Size*Category*Method & 0.942 & 2.283 & 2 & 0.319 \\
\hline
\end{tabular}

Table 5.6: Results of within subjects effects test. The main effects of factors with significant value smaller than 0.05 are considered as significant.

\begin{tabular}{lrrrr}
\hline Factor & df & Mean Square & F & Sig. \\
\hline Size & 1 & 437.008 & 336.437 & 0 \\
Error(Size) & 39 & 1.299 & & \\
Category & 1 & 2.133 & 1.092 & 0.302 \\
Error(Category) & 39 & 1.954 & & \\
Method & 1.818 & 342.033 & 58.209 & 0 \\
Error(Method) & 70.918 & 5.876 & & \\
Size * Category & 1 & 7.008 & 6.722 & 0.013 \\
Error(Size*Category) & 39 & 1.043 & & \\
Size * Method & 2 & 5.515 & 5.712 & 0.005 \\
Error(Size*Method) & 78 & 0.965 & & \\
Category * Method & 1.746 & 8.534 & 8.839 & 0.001 \\
Error(Category*Method) & 68.11 & 0.966 & & \\
\hline
\end{tabular}

Huynh-Feldt method, in which the degrees of freedom of the factors with violation are reduced, thereby making the significant value of the F-ratio more conservative.

Table 5.6 shows the test results of within-subjects effects of content size, image category, precompensation method and interactions between them. From the results, it is found that the effect of precompensation method on the recognition 
accuracy was significant, $F(1.82,70.92)=58.2, p<0.01$. This indicates the recognition accuracy of the subjects significantly varied when different compensation methods were applied to the test images. The effect of content size of the image on the recognition accuracy was significant, $F(1,39)=336.4, p<0.01$. The reason for this significance is quite intuitive, as the recognition of large letters or icons was less disrupted by the degradation caused by the ocular aberration. The main effect of the image category (icon or letter) was found not significant, $F(1,39)=2.1, p>0.05$, which indicates that there were no significant differences in the recognition difficulty of icons and letters.

The significance of main effect of precompensation method only confirmed the performance differences existed between NPC, SPC and DPC. To compare their visual performance, pairwise comparisons were performed between the three precompensation methods, with the results tabulated in Table 5.7. From the comparison results, it was found that the difference between DPC and NPC is significant, $p<0.01$. This means that the recognition accuracy when dynamic precompensation was applied was significantly higher than the accuracy when no precompensation was applied, thus confirming the visual benefit achieved by the dynamic precompensation. Likewise, the difference between DPC and SPC is also found significant, $p<0.01$. This significance verifies the necessity of dynamic update of image compensation, as the visual performance of the subjects was significantly improved when the precompensation method was switched from SPC to DPC. Interestingly, the difference between SPC and NPC was not significant. This suggests that, due to the lack of compensation adjustment for new pupil sizes, the desired visual benefit of static precompensation was ruined by the mismatch between the fixed measured aberration and the actual aberration at the time of the recognition test. In other 
Table 5.7: Pairwise comparisons between three precompensation methods. The mean difference of two methods is significant at the 0.05 level.

\begin{tabular}{ccccccc}
\hline & & & & \multicolumn{3}{c}{$95 \%$ Confidence Interval } \\
\hline Method(I) & Method (J) & Mean Difference & Std. & Sig. & Lower Bound & Upper Bound \\
\hline \multirow{2}{*}{ NPC } & SPC & 0.656 & 0.303 & 0.11 & -0.102 & 1.415 \\
& DPC & -2.019 & 0.241 & 0 & -2.622 & -1.415 \\
\multirow{2}{*}{ SPC } & NPC & -0.656 & 0.303 & 0.11 & -1.415 & 0.102 \\
& DPC & -2.675 & 0.224 & 0 & -3.235 & -2.115 \\
\multirow{2}{*}{ DPC } & NPC & 2.019 & 0.241 & 0 & 1.415 & 2.622 \\
& SPC & 2.675 & 0.224 & 0 & 2.115 & 3.235 \\
\hline
\end{tabular}

words, applying static precompensation did not help the subjects perform better than the test images presented without any preprocessing.

The interaction between content size and compensation method was found significant, $F(2,78)=5.7, p<0.01$. This reflects that the improvement of recognition accuracy for large test images was not as high as for small ones, when the precompensation was applied. This significance can be explained by the fact that most of the test images with large size could be recognized correctly by the subjects, regardless of the compensation method was applied or not, whereas a large proportion of test image with small size could not be recognized without appropriate precompensation.

More interestingly, the interaction between image category and precompensation method was also found significant, $F(1.75,68.1)=8.84, p<0.01$. Consistent with this significance, it is found the accuracy improvement for the icons was lower than for the letters, when dynamic precompensation was applied. This is probably because the icons were more vulnerable to the ringing artifacts introduced in the process of precompensation, as the test images with icons had more complicated shapes and edges than the letters. 


\subsubsection{Results of Subjective Assessments}

In the questionnaire, most of the subjects (18 of 20) reported that the sharpness of the test images was increased with dynamic precompensation, comparing to the original images. The remaining two subjects claimed that the sharpness of the test images with DPC and NPC were at the same level. In addition, most of the subjects (16 of 20) reported that, in comparison to the test images with SPC, the test images with DPC appeared sharper and clearer. The answers to these two questions were consistent with the results of the statistical analysis, further confirming the benefit of the dynamic precompensation method.

Interestingly, most of the subjects (17 of 20) claimed that the test images with letters were more easily recognized than the test images with icons. Two of the remaining three subjects claimed equivalent recognition difficulty and only one subject stated that the test images with icons were more easily recognized. The subjects' answers to this question are contrary to the results of statistical analysis, in which

the test images with letters and icons were found to have no significant differences in recognition difficulty.

Although the recognition accuracy was considerably improved by the dynamic precompensation method, the vision quality after applying the dynamic precompensation was still far from fully satisfactory. $55 \%$ of the subjects (11 of 20) reported that the problem that most impeded their correct recognition of images after precompensation was the low contrast of the test images. In contrast, another $25 \%$ of the subjects ( 5 of 20 ) pointed to the ringing artifacts and the remaining $20 \%$ of the subjects (4 of 20) pointed to other factors (e.g., abnormal blur and edge overlapping). The answers to this question are shown in Figure 5.7 as a pie chart. Obviously, the benefit of our dynamic precompensation method was mainly limited 

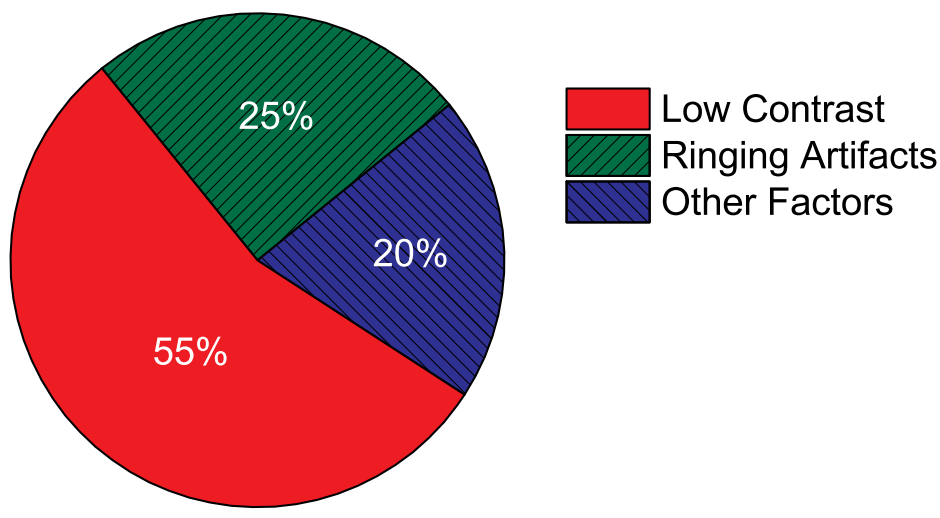

Figure 5.7: Problems that most impeded recognition of the test images with dynamic precompensation. 11 subjects reported low contrast. 5 subjects reported ringing artifacts and other 4 subjects reported other factors.

by the problems of low contrast and ringing artifacts, which were also demonstrated in the software simulation and artificial eye experiments.

\subsubsection{Discussion}

In the human subject experiment above, we verified the effectiveness of our dynamic precompensation method in a realistic viewing environment of computer use. However, an average of about $26 \%$ of the test images still could not be correctly recognized by the subjects, even after the dynamic image precompensation was applied. The problems of precompensation were also revealed in the subjective assessment from the subjects, mainly reported as low contrast and ringing artifacts. In practice, the interpretation of the results from the image recognition test can be more complicated. After the test, a few subjects reported that they preferred viewing the original images rather than the precompensated images, even though they admitted that the test images with compensation had much sharper edges. Interestingly, it seems that the image blur caused by the ocular aberrations was more acceptable than the possible distortion introduced in the precompensation process, even 
if the distortion caused by the precompensation was much milder. Some subjects indicated they felt more familiar and comfortable with the regular blurring pattern of the refractive errors like myopia and astigmatism. While other study indicated that visual search of icons is quite resistant to small or moderate deterioration of image quality [57], it seems that some subjects in our experiment could identify the test images correctly based on visual experience, even if the test images had been severely degraded. On the other hand, some subjects indicated that the test images with precompensation were "weird" and an adaptation process was required before they became comfortable with the compensated image, especially when the static precompensation was applied.

As in the case of image restoration, the image precompensation process is also subject to the problems of ringing artifacts and noise amplification [4]. The problem of ringing artifacts is mainly caused by the regularization error distributed on the whole spectrum, usually becoming evident around the regions with abrupt intensity transitions. The problem of noise amplification is introduced in the process of inverse Blurring filtering (IBF), adding extra degradation into the retinal image. The noise in the image precompensation process primarily comes from the measurement of the ocular aberration and the display devices. For the computer users with low vision, the degradation introduced by the noise may not be critical since the amplified noise will be restrained by the viewing process. In other words, people with low vision may not be able to perceive the amplified noise due to the low pass nature of the aberrated eyes. In the case of image precompensation, the image quality of the retinal image is also related to the accuracy of the precompensation model. More specifically, the ocular aberration that is used to generate compensation can not be perfectly equivalent to the actual aberration. In the image recognition test, some subjects reported that the precompensated images appeared more clearly at some 
certain isolated moments during viewing. This is because the resized aberration used to generate the image precompensation at those moments was closer to the actual one. The problem of ringing artifacts is in some extent conflicted with the problem of low contrast [27]. By adding the tolerance to the regularization error, the contrast of the precompensated image can be increased. Conversely, if we disregard the contrast reduction after precompensation, the influence of ringing artifacts can be reduced to unnoticeable levels. Thus, the biggest new challenge that emerges from image precompensation is the reduction of contrast after precompensation. For image restoration, such contrast loss problem does not exist, as the restored images have no physical limitation on intensity. With regular display devices, due to the limited intensity range, the contrast loss after precompensation is unavoidable if retinal images with the same sharpness as the original images are desired. Thus, one more practical strategy is improving the contrast of precompensated images while keeping most of the fidelity of the original images, which is more helpful to the computer users with low vision.

\subsection{Summary}

In this chapter, the factorial design and the protocol of the human subject experiment were introduced. The improvement of visual performance achieved by the image precompensation was evaluated though image recognition tests performed by the subjects. The accuracy of subjects to recognize the test images with static precompensation (SPC), dynamic precompensation (DPC) and no precompensation (NPC) were compared through statistical analysis. The interactions between the image category, content size and precompensation method were also investigated. The results of statistical analysis show significant improvement of the recognition 
accuracy when dynamic precompensation was applied, comparing to both of the images with static precompensation and original images without precompensation. The subjective assessment collected from subjects was also analyzed, confirming the effectiveness of the dynamic image precompensation method. 


\section{CHAPTER 6 \\ CONCLUSIONS AND FUTURE WORK}

\subsection{Concluding Remarks}

This dissertation presented an image-processing-based precompensation method, for improving the visual performance of the computer user with ocular aberrations. In this method, personalized and dynamic precompensation is applied to the images, before they are presented on the screen, according to the real-time ocular aberration of the observer. The update of the image precompensation model, which is required because of the dynamic features of the ocular aberration, is performed according to the pupil size variations in the eye of the user.

The potential visual benefit of the dynamic image precompensation method was explored through a software simulation, in which the aberration data was from a real human subject. An artificial eye experiment was then implemented, in order to evaluate the effective retinal image quality after precompensation, in an objective manner. We also conducted a human subject experiment to verify the effectiveness of the dynamic precompensation method through an image recognition test, in a realistic environment of computer use. The results of the human subject experiment revealed that the visual performance of the subjects was significantly improved after the dynamic precompensation was applied. Consistent with the artificial eye experiment, the statistical analysis and subjective assessment from the experiment participants confirmed the necessity of the dynamic update of the precompensation model according to the real-time aberration.

The major contributions of this dissertation are summarized in three aspects: modeling of the computer user's retinal image, dynamic precompensation and demonstration of an application prototype. 


\subsubsection{Modeling of Computer User's Retinal Image}

The modeling of the retinal image formation of the computer user is the foundation of our image precompensation method. In this dissertation, we presented a complete and systematic modeling approach to describe the viewing process of the computer users, taking advantage of key concepts, which include Zernike polynomials, wavefront aberration, Point Spread Function (PSF) and Modulation Transfer Function (MTF). The computer user's ocular aberration (including low order aberrations and high order aberrations), viewing distance, field of view and pupil size were taken into consideration and involved, when the image viewing model was established. This model can be used to describe the retinal image quality of the computer users in an accurate and robust way. Excluding the neural processing of the human visual system, this model can also be used to predict the image perception of the computer users when viewing the images presented on the computer screen. More importantly, this model makes it possible to compensate the computer user's ocular aberration and the image degradation caused by it in a proactive way, through the preprocessing of the images to be presented.

\subsubsection{Dynamic Precompensation}

The ultimate goal of this study is developing an image preprocessing method to relieve the visual blurring of the computer users with low vision or ocular aberrations. The accurate aberrations of the computer user's eyes can be measured and quantified through wavefront sensing device, which are usually reported as a set of Zernike coefficients. The specific pattern of visual blurring involved in image viewing is accessible in analytical representations, along with the modeling of the retinal image formation of the observer's eye. Thus, in order to overcome the visual 
blurring, precompensation with a "reverse blurring" process was applied to the images before their presentation on the screen, aiming to counteract the impact of the original visual blurring.

The precompensation applied on the images has to be personalized according to the computer user's ocular aberration, since the ocular aberration of each human eye is unique and mismatched precompensation will cause degradation in the visual performance. However, even for a given eye of a specific person, the ocular aberration can not be described statically. Due to the dynamic features of the eye's optical system, the aberration of the eye varies all the time, making the static personalized precompensation inadequate. In the context of computer use, the ocular aberration is most influenced by the pupil size. In this dissertation, the precompensation was generated and updated according to the dynamic ocular aberration of the computer user, which was obtained by the resizing of the original wavefront aberration measured to smaller pupil sizes. The accuracy and reliability of the resized aberration was verified by comparing it with the aberration measured under the same pupil size, with the same pupil diameter. In addition, a histogram side-clipping method was developed and integrated into the compensating process, to relieve the contrast loss problem after precompensation.

\subsubsection{Prototype of Application}

In this dissertation, an application prototype of the personalized and dynamic precompensation system was demonstrated. In the prototype system, the referential wavefront aberration was measured through the wavefront aberrometer and recorded in a personal file as Zernike coefficients. In practice, this personal aberration file can be even stored in a flash drive and imported into the system in advance of the 
application of the precompensation. When the images were requested to present, the precompensation was applied according to the resized aberration, which was computed in real-time. One important merit of the precompensation system is that it is automated and parameterless. Thus, no manual intervention is needed when the precompensation system is running. Except for the need of an eye-tracker, the precompensation system can be implemented with regular personal computers and display devices. To reduce the cost, the eye-tracker could, prospectively, be replaced by a high definition camera, since only the pupil diameter is required. The prototype system developed has been applied in the image recognition test, providing evaluation to the effectiveness of the precompensation. Even though the prototype system is still preliminary, it lays a good foundation for more specific applications of the dynamic precompensation method.

\subsection{Limitations}

It has been verified that the dynamic precompensation method is able to significantly improve the visual performance of the computer users with ocular aberrations. However, the potential of the method has not been fully realized due to several limitations.

Firstly, the image quality perceived by the observer is limited by the low contrast problem, when the precompensation is applied. Although the contrast loss after precompensation is reduced by the developed histogram clipping method, the perceived contrast is still far from optimal. The essential reason for the contrast loss is that the calculated intensity range of the precompensated image is often beyond the display capacity of regular display devices, which only have limited intensity levels. While the explanation to this problem is straightforward, finding a solution to it is quite 
challenging. So far, we have not found effective and universal approaches to improve the contrast of perceived images with precompensation to a fully satisfactory level. Thus, this is still an open problem that needs further investigation.

Secondly, the wavefront aberration used to establish the precompensation model in this study is monochromatic. This had little impact on the image quality in our prototype system, as the test images used were all monochrome. However, in a practical context of computer use, in order to obtain a more accurate precompensation model, chromatic aberration should be used instead. The precompensation of chromatic image is coupled to the problem of contrast loss. In the process of intensity shifting and downscaling before the display of the precompensated image, color information of the image will be also distorted, along with the reduction of contrast. Thus, preserving the color information in the precompensation may also prove to be a challenging problem.

Lastly, in this study, the dynamic precompensation method was designed and evaluated based on monocular vision. Obviously, this could limit the direct application of the dynamic precompensation in realistic scenarios of computer use. Thus, precompensation model based on binocular vision may need to be developed.

\subsection{Future Directions}

One important future work direction is to improve the contrast of the perceived image after the precompensation. While new display devices or new display approaches will be investigated, aiming to fully preserve the contrast in the process of precompensation, more practical and feasible improvement of contrast may probably be obtained in a trade-off with the fidelity of the image. By sacrificing some of the fidelity of the image, the contrast of the precompensated image can be en- 
hanced to a satisfactory level. In addition, in order to generate precompensation with "partial" aberration correction, new precompensation algorithms other than inverse Blurring filtering need to be developed. This partial aberration correction may be quite helpful to some computer users with low vision.

Another possible future direction is to develop precompensation models based on the binocular vision of the computer user. As the ocular aberration of the left eye and the right eye of the same person are generally different, precompensation models established based on either one of them will not be appropriate for the other. Thus, both of the ocular aberrations of the two eyes should be considered, to generate a binocular precompensation approach, which may require a deep understanding of binocular nature of the human visual system. 


\section{BIBLIOGRAPHY}

[1] M. Alonso, A. Barreto, J. Cremades, J. Jacko, and M. Adjouadi. Image precompensation to facilitate computer access for users with refractive errors. Behaviour $\&$ Information Technology, 24:161-173, 2005.

[2] M. Alonso, A. Barreto, and J. G. Cremades. Image pre-compensation to facilitate computer access for users with refractive errors. In Proc. ACM SIGACCESS conf. Comp. Access., pages 126-132, 2004.

[3] W. Atabany and P. Degenaar. A robust edge enhancement approach for low vision patients using scene simplification. In IEEE Biomedical Engineering Conference, Cairo International, pages 1-4, 2008.

[4] M. R. Banham and A. K. Katsaggelos. Digital image restoration. IEEE Signal Process. Mag., 14(2):24-41, Mar. 1997.

[5] P. Blenkhorn, G. Evans, A. King, S. Hastuti Kurniawan, and A. Sutcliffe. Screen magnifiers: evolution and evaluation. IEEE Computer Graphics and Applications, 23(5):54-61, 2003.

[6] J. T. Cacioppo, L. G. Tassinary, and G. Berntson. Handbook of psychophysiology. Cambridge University Press, 2007.

[7] C. E. Campbell. Matrix method to find a new set of zernike coefficients from an original set when the aperture radius is changed. J. Opt. Soc. Am. A., 20(2):209-217, 2003.

[8] W. Charman and G. Heron. Fluctuations in accommodation: a review. Ophthalmic and Physiological Optics, 8(2):153-164, 1988.

[9] M. Collins, B. Davis, and J. Wood. Microfluctuations of steady-state accommodation and the cardiopulmonary system. Vision research, 35(17):2491-2502, 1995.

[10] N. Congdon, B. O'colmain, C. Klaver, R. Klein, B. Munoz, D. Friedman, J. Kempen, H. Taylor, P. Mitchell, et al. Causes and prevalence of visual impairment among adults in the united states. Archives of ophthalmology, 122(4):477-485, 2004.

[11] G. Dai. Scaling zernike expansion coefficients to smaller pupil sizes: a simpler formula. J. Opt. Soc. Am. A., 23(3):539-543, 2006. 
[12] G. Dai. Wavefront optics for vision correction. SPIE, 2008.

[13] A. T. Duchowski. Eye tracking methodology: Theory and practice, volume 373. Springer, 2007.

[14] E. J. Fernández, I. Iglesias, and P. Artal. Closed-loop adaptive optics in the human eye. Optics letters, 26(10):746-748, 2001.

[15] A. Field. Discovering statistics using SPSS. Sage Publications Limited, 2009.

[16] E. M. Fine and E. Peli. Enhancement of text for the visually impaired. J. Opt. Soc. Am. A., 12(7):1439-1447, 1995.

[17] G. Fischer. User modeling in human-computer interaction. User modeling and user-adapted interaction, 11(1-2):65-86, 2001.

[18] C. Gena. Methods and techniques for the evaluation of user-adaptive systems. The knowledge engineering review, 20(01):1-37, 2005.

[19] A. Glasser, M. Campbell, et al. Presbyopia and the optical changes in the human crystalline lens with age. Vision research, 38(2):209-229, 1998.

[20] R. C. Gonzalez and E. Richard. Digital Image Processing. Prentice Hall, second edition, 2002.

[21] J. Goodman. Introduction to Fourier optics. McGraw-hill, 2008.

[22] W. W. Grings and M. E. Dawson. Emotions and bodily responses: A psychophysiological approach. Academic Press New York, 1978.

[23] A. Guirao, J. Porter, D. R. Williams, and I. G. Cox. Calculated impact of higher-order monochromatic aberrations on retinal image quality in a population of human eyes: erratum. J. Opt. Soc. Am. A., 19(3):620-628, 2002.

[24] E. H. Hess. The tell-tale eye: How your eyes reveal hidden thoughts and emotions. Van Nostrand Reinhold New York, 1975.

[25] H. Hofer, P. Artal, B. Singer, J. Aragón, and D. R. Williams. Dynamics of the eye's wave aberration. J. Opt. Soc. Am. A., 18(3):497-506, 2001. 
[26] H. C. Howland and B. Howland. A subjective method for the measurement of monochromatic aberrations of the eye. J. Opt. Soc. Am. A., 67(11):1508-1518, 1977.

[27] J. Huang, A. Barreto, and M. Adjouadi. Evaluation of dynamic image precompensation for computer users with severe refractive error. In Proceedings of the 14th International ACM SIGACCESS Conference on Computers and Accessibility, pages 175-182, 2012.

[28] J. Huang, A. Barreto, M. Alonso, and M. Adjouadi. Contrast improvement in pre-compensation of ocular aberrations for computer users. Biomedical sciences instrumentation, 48:179-186, 2012.

[29] S. T. Iqbal, X. S. Zheng, and B. P. Bailey. Task-evoked pupillary response to mental workload in human-computer interaction. In CHI'04 extended abstracts on Human factors in computing systems, pages 1477-1480. ACM, 2004.

[30] D. R. Iskander, M. J. Collins, M. R. Morelande, and M. Zhu. Analyzing the dynamic wavefront aberrations in the human eye. IEEE Trans. Biomed. Eng., 51:1969-1980, Nov. 2004.

[31] J. A. Jacko, R. H. Rosa Jr, I. U. Scott, C. J. Pappas, and M. A. Dixon. Visual impairment: The use of visual profiles in evaluations of icon use in computerbased tasks. International Journal of Human-Computer Interaction, 12(1):151164, 2000 .

[32] R. J. Jacob and K. S. Karn. Eye tracking in human-computer interaction and usability research: Ready to deliver the promises. Mind, 2(3):573-605, 2003.

[33] M. P. Janisse. Pupillometry: The psychology of the pupillary response. Hemisphere Publishing Corporation Washington, 1977.

[34] T.-L. Ji, M. Sundareshan, and H. Roehrig. Adaptive image contrast enhancement based on human visual properties. IEEE Transactions on Medical Imaging, 13(4):573-586, 1994.

[35] M. Juris and M. Velden. The pupillary response to mental overload. Physiological Psychology, 1977.

[36] F. Karray, M. Alemzadeh, J. A. Saleh, and M. N. Arab. Human-computer interaction: Overview on state of the art. International Journal on Smart Sensing and Intelligent Systems, 1(1):137-159, 2008. 
[37] A. K. Katsaggelos, J. Biemond, R. W. Schafer, and R. M. Mersereau. A regularized iterative image restoration algorithm. IEEE Trans. Signal Process., 39(4):914-929, Apr. 1991.

[38] M. P. Keating. Geometric, physical, and visual optics. Butterworth-Heinemann Boston, 2002.

[39] J. P. Keener and J. Sneyd. Mathematical physiology, volume 8. Springer, 1998.

[40] L. M. Kennedy and M. Basu. Image enhancement using a human visual system model. Pattern Recognition, 30(12):2001-2014, 1997.

[41] J. Kim, A. Vora, and E. Peli. Mpeg-based image enhancement for the visually impaired. Optical Engineering, 43:1318, 2004.

[42] R. R. Krueger, R. A. Applegate, and S. MacRae. Wavefront customized visual corrections: the quest for super vision II. Slack Thorofare, 2004.

[43] T. A. Lawton, J. Sebag, A. A. Sadun, and K. R. Castleman. Image enhancement improves reading performance in age-related macular degeneration patients. Vision research, 38(1):153-162, 1998.

[44] T. B. Lawton. Image enhancement filters significantly improve reading performance for low vision observers. Ophthalmic and Physiological Optics, 12(2):193200, 1992.

[45] S. J. Leat, G. Omoruyi, A. Kennedy, and E. Jernigan. Generic and customised digital image enhancement filters for the visually impaired. Vision research, 45(15):1991-2007, 2005.

[46] J. Liang, B. Grimm, S. Goelz, J. F. Bille, et al. Objective measurement of wave aberrations of the human eye with the use of a hartmann-shack wavefront sensor. J. Opt. Soc. Am. A., 11(7):1949-1957, 1994.

[47] J. Liang and D. R. Williams. Aberrations and retinal image quality of the normal human eye. J. Opt. Soc. Am. A., 14(11):2873-2883, 1997.

[48] J. Liang, D. R. Williams, and D. T. Miller. Supernormal vision and highresolution retinal imaging through adaptive optics. J. Opt. Soc. Am. A., 14(11):2884-2892, 1997. 
[49] S. MacRae, J. Schwiegerling, and R. Snyder. Customized corneal ablation and super vision. Journal of Refractive Surgery, 16(2):230-235, 2000.

[50] J. G. May, R. S. Kennedy, M. C. Williams, W. P. Dunlap, and J. R. Brannan. Eye movement indices of mental workload. Acta Psychologica, 75(1):75-89, 1990.

[51] Kronkosky Charitable Foundation. Visual impairment and blindness, research brief, 2012.

[52] The Arlene R. Gordon Research Institute, Lighthouse International. Statistics on vision impairment. http://www.lighthouse.org/research/ statistics-on-vision-impairment/. Accessed: 2013-04-01.

[53] Tobii, Inc. Tobii t60 \& t120 eye tracker user manul. http: //www.tobii.com/Global/Analysis/Downloads/User_Manuals_and_ Guides/Tobii_T60_T120_EyeTracker_UserManual.pdf. Accessed: 2013-0426.

[54] Wavefront Sciences, Inc. Coas-hdtm model 2800 high definition wavefront aberrometer. http: //www . wavef rontsciences .com/COAS-HD.pdf. Accessed: 2013-04-26.

[55] World Health Orgnization. Visual impairment and blindness. http://www . who.int/mediacentre/factsheets/fs282/en/. Accessed: 2013-04-01.

[56] B. A. Myers. A brief history of human-computer interaction technology. interactions, 5(2):44-54, 1998.

[57] R. Näsänen and H. Ojanpää. Effect of image contrast and sharpness on visual search for computer icons. Displays, 24(3):137-144, 2003.

[58] R. Navarro, E. Moreno, and C. Dorronsoro. Monochromatic aberrations and point-spread functions of the human eye across the visual field. J. Opt. Soc. Am. A., 15(9):2522-2529, 1998.

[59] T. Oshika, K. Miyata, T. Tokunaga, T. Samejima, S. Amano, S. Tanaka, Y. Hirohara, T. Mihashi, N. Maeda, and T. Fujikado. Higher order wavefront aberrations of cornea and magnitude of refractive correction in laser in situ keratomileusis. Ophthalmology, 109(6):1154-1158, 2002. 
[60] T. Partala and V. Surakka. Pupil size variation as an indication of affective processing. International journal of human-computer studies, 59(1):185-198, 2003.

[61] S. Patel, J. Marshall, and F. Fitzke. Refractive index of the human corneal epithelium and stroma. Journal of refractive surgery, 11(2):100-105, 1995.

[62] E. Peli. Image enhancement for the visyally impaired. Optical Engineering, 23(1):47-51, 1984.

[63] E. Peli. Recognition performance and perceived quality of video enhanced for the visually impaired. Ophthalmic and Physiological Optics, 25(6):543-555, 2005 .

[64] E. Peli, J. Kim, Y. Yitzhaky, R. B. Goldstein, and R. L. Woods. Wideband enhancement of television images for people with visual impairments. J. Opt. Soc. Am. A., 21(6):937-950, 2004.

[65] E. Peli, E. Lee, C. L. Trempe, and S. Buzney. Image enhancement for the visually impaired: the effects of enhancement on face recognition. J. Opt. Soc. Am. A., 11(7):1929-1939, 1994.

[66] T. Peli and J. S. Lim. Adaptive filtering for image enhancement. Optical Engineering, 21(1):108-118, 1982.

[67] S. M. Pizer, E. P. Amburn, J. D. Austin, R. Cromartie, A. Geselowitz, T. Greer, B. ter Haar Romeny, J. B. Zimmerman, and K. Zuiderveld. Adaptive histogram equalization and its variations. Computer vision, graphics, and image processing, 39(3):355-368, 1987.

[68] B. C. Platt. History and principles of shack-hartmann wavefront sensing. Journal of Refractive Surgery, 17(5):573-577, 2001.

[69] G. K. Poock. Information processing vs pupil diameter. Perceptual and Motor Skills, 37(3):1000-1002, 1973.

[70] J. Porter, A. Guirao, I. G. Cox, and D. R. Williams. Monochromatic aberrations of the human eye in a large population. J. Opt. Soc. Am. A., 18(8):1793-1803, 2001. 
[71] R. B. Rabbetts. Bennett \& Rabbetts' clinical visual optics. ButterworthHeinemann Edinburgh, UK, 1998.

[72] P. Ren, A. Barreto, Y. Gao, and M. Adjouadi. Affective assessment by digital processing of the pupil diameter. IEEE Transactions on Affective Computing, $4(1): 2-14,2013$.

[73] W. H. Richardson. Bayesian-based iterative method of image restoration. J. Opt. Soc. Am., 62(1):55-59, 1972.

[74] A. Roorda. Adaptive optics for studying visual function: A comprehensive review. Journal of Vision, 11(5):1-21, 2011.

[75] L. I. Rudin, S. Osher, and E. Fatemi. Nonlinear total variation based noise removal algorithms. Physica D: Nonlinear Phenomena, 60(1-4):259-268, 1992.

[76] R. Sabesan, T. M. Jeong, L. Carvalho, I. G. Cox, D. R. Williams, and G. Yoon. Vision improvement by correcting higher-order aberrations with customized soft contact lenses in keratoconic eyes. Optics letters, 32(8):1000-1002, 2007.

[77] L. Sawides, E. Gambra, D. Pascual, C. Dorronsoro, and S. Marcos. Visual performance with real-life tasks under adaptive-optics ocular aberration correction. Journal of Vision, 10(5):1-12, 2010.

[78] J. Schwiegerling. Scaling zernike expansion coefficients to different pupil sizes. J. Opt. Soc. Am. A., 19(10):1937-1945, 2002.

[79] A. Sears and J. A. Jacko. The human-computer interaction handbook: fundamentals, evolving technologies and emerging applications. CRC Press, 2007.

[80] H. R. Sheikh and A. C. Bovik. Image information and visual quality. IEEE Transactions on Image Processing, 15(2):430-444, 2006.

[81] S. R. Steinhauer, G. J. Siegle, R. Condray, and M. Pless. Sympathetic and parasympathetic innervation of pupillary dilation during sustained processing. International Journal of Psychophysiology, 52(1):77-86, 2004.

[82] J. Tang, J. Kim, and E. Peli. Image enhancement in the jpeg domain for people with vision impairment. IEEE Trans. Biomed. Eng., 51(11):2013-2023, 2004. 
[83] D. Te'eni, J. M. Carey, and P. Zhang. Human-computer interaction: Developing effective organizational information systems. John Wiley \& Sons, 2005.

[84] L. Thibos. Formation and sampling of the retinal image. In K. K. D. Valiois, editor, Seeing. Academic Press, second edition, 2000.

[85] L. Thibos, A. Bradley, D. Still, X. Zhang, and P. Howarth. Theory and measurement of ocular chromatic aberration. Vision Research, 30(1):33-49, 1990.

[86] L. Thibos, A. Bradley, and X. Zhang. Effect of ocular chromatic aberration on monocular visual performance. Optometry \& Vision Science, 68(8):599-607, 1991.

[87] L. N. Thibos, R. A. Applegate, J. T. Schwiegerling, R. Webb, et al. Standards for reporting the optical aberrations of eyes. Vision science and its applications, 35:232-244, 2000.

[88] S. Vitale, R. D. Sperduto, and F. L. Ferris III. Increased prevalence of myopia in the united states between 1971-1972 and 1999-2004. Arch. Ophthalmol., 127(12):1632-1639, 2009.

[89] G. Walsh, W. N. Charman, and H. C. Howland. Objective technique for the determination of monochromatic aberrations of the human eye. J. Opt. Soc. Am. A., 1(9):987-992, 1984.

[90] L. Wang, E. Dai, D. D. Koch, and A. Nathoo. Optical aberrations of the human anterior cornea. Journal of Cataract \& Refractive Surgery, 29(8):15141521, 2003.

[91] Z. Wang and A. C. Bovik. A universal image quality index. IEEE Signal Processing Letters, 9(3):81-84, 2002.

[92] Z. Wang, A. C. Bovik, H. R. Sheikh, and E. P. Simoncelli. Image quality assessment: From error visibility to structural similarity. IEEE Transactions on Image Processing, 13(4):600-612, 2004.

[93] R. H. Webb, C. M. Penney, and K. P. Thompson. Measurement of ocular local wavefront distortion with a spatially resolved refractometer. Applied Optics, 31(19):3678-3686, 1992. 
[94] D. R. Williams. Topography of the foveal cone mosaic in the living human eye. Vision Research, 28(3):433-454, 1988.

[95] D. R. Williams, D. H. Brainard, M. J. McMahon, and R. Navarro. Double-pass and interferometric measures of the optical quality of the eye. J. Opt. Soc. Am. A., 11(12):3123-3135, 1994.

[96] J. S. Wolffsohn, D. Mukhopadhyay, and M. Rubinstein. Image enhancement of real-time television to benefit the visually impaired. American journal of ophthalmology, 144(3):436-440, 2007.

[97] G.-Y. Yoon and D. R. Williams. Visual performance after correcting the monochromatic and chromatic aberrations of the eye. J. Opt. Soc. Am. A., $19(2): 266-275,2002$. 
VITA

\title{
JIAN HUANG
}

\author{
2002-2006 \\ B.S., Electrical Engineering \\ Wuhan University of Science and Technology \\ Wuhan, China \\ 2006-2009 \\ M.S., Computer Engineering \\ Wuhan University of Science and Technology \\ Wuhan, China \\ 2009-present \\ Ph.D. Candidate, Electrical Engineering \\ Florida International University \\ Miami, Florida
}

\section{PUBLICATIONS AND PRESENTATIONS}

J. Huang, A. Barreto, P. Ren and M. Adjouadi, "Personalized and dynamic image precompensation for computer users with ocular aberrations," Behaviour \& Information Technology, accepted for publication.

P. Ren, A. Barreto, J. Huang, Y. Gao, F. R. Ortega, and M. Adjouadi, "Offline and online stress detection through processing pupil diameter signal," Annals of Biomedical Engineering, under review.

J. Huang, A. Barreto, M. Alonso Jr., and M. Adjouadi, "Image pre-compensation for visually impaired computer users with variable pupil size," Emerging Trends in Computing, Informatics, Systems Sciences, and Engineering, Vol. 151, pp. 171182, 2013, Springer.

J. Huang, A. Barreto, M. Alonso Jr., and M. Adjouadi, "Contrast enhancement in image pre-compensation for computer users with visual aberrations," Innovations and Advances in Computer, Information, Systems Sciences, and Engineering, Vol. 152, pp. 371-380, 2013, Springer.

J. Huang, A. Barreto, M. Alonso Jr., and M. Adjouadi, "Contrast improvement in pre-compensation of ocular aberrations for computer users," Biomedical Sciences Instrumentation, vol. 48, pp. 179-186, 2012.

J. Huang, A. Barreto and M. Adjouadi, "Evaluation of dynamic image pre-compensation for computer users with severe refractive error," Proceedings of ACM SIGACCESS Conference on Computers and Accessibility, pp. 175-182, 2012.

J. Huang, A. Barreto, and M. Adjouadi, "Dynamic image pre-compensation for 
computer access by individuals with ocular aberrations," Proceedings of IEEE Engineering in Medicine and Biology Society Conference, EMBC, pp. 3320-3323, 2012.

J. Huang, A. Barreto, M. Alonso Jr., and M. Adjouadi, "Vision correction for computer users based on image pre-compensation with changing pupil size," Proceedings of IEEE Engineering in Medicine and Biology Society Conference, EMBC, pp. 4868-4871, 2011. 